\title{
Aluminum-Catalyzed Enantio- and Diastereoselective Carbonyl Addition of Propargylsilanes. A New Approach to Enantio-enriched Vinyl Epoxides
}

\author{
David A. Evans* and Yimon Aye \\ Department of Chemistry \& Chemical Biology, Harvard University, Cambridge, Massachusetts 02138
}

\section{Supporting Information}

General Information. All reactions were carried out under an atmosphere of argon or nitrogen in ovendried glassware with magnetic stirring. THF, toluene, ether, and $\mathrm{CH}_{2} \mathrm{Cl}_{2}$ were purified by passage through a bed of activated alumina. ${ }^{i}$ Solvents and reagents were purified prior to use following the guidelines of Perrin and Armarego. ii Purification of reaction products was carried out by flash chromatography using EM Reagent silica gel 60 (230-400 mesh). Analytical thin layer chromatography was performed on EM Reagent $0.25 \mathrm{~mm}$ silica gel 60-F plates. Visualization was accomplished with UV light and either potassium permanganate stain or ceric ammonium nitrate stain followed by heating. Optical rotations were measured on a Jasco DIP-0181 digital polarimeter with a sodium lamp and are reported as follows: $[\alpha]_{\mathrm{D}}{ }^{\mathrm{T}}{ }^{\circ} \mathrm{C}(c \mathrm{~g} / 100 \mathrm{~mL}$, solvent). Infrared spectra were recorded on a Perkin Elmer 1600 series FT-IR spectrometer. ${ }^{1} \mathrm{H}$ NMR spectra were recorded on a Varian Inova-500 (500 MHz) or Varian Mercury-400 (400 MHz) spectrometer and are reported in ppm using solvent as an internal standard $\left(\mathrm{CDCl}_{3}\right.$ at $7.26 \mathrm{ppm}$ or $\mathrm{C}_{6} \mathrm{D}_{6}$ at $\left.7.33 \mathrm{ppm}\right)$. Data reported as $(\mathrm{s}=$ singlet, $\mathrm{d}=\mathrm{doublet}$, $\mathrm{t}=$ triplet, q=quartet, $\mathrm{s}$,=sextet, $\mathrm{h}=$ heptet, $\mathrm{o}=$ octet, $\mathrm{m}=$ multiplet, brs=broad singlet; integration; coupling constant(s) in $\mathrm{Hz}$ ). Proton-decoupled ${ }^{13} \mathrm{C}$ NMR spectra were recorded on Varian Inova-600 (150 MHz), Varian Inova-500 (125 MHz) and Varian Mercury-400 (100 MHz) spectrometers and are reported in ppm using solvent as an internal standard $\left(\mathrm{CDCl}_{3}\right.$ at $77.0 \mathrm{ppm}$, or $\mathrm{C}_{6} \mathrm{D}_{6}$ at $\left.128.3 \mathrm{ppm}\right)$. High resolution mass spectra were obtained on Jeol AX-505 or SX-102 spectrometers at the Harvard University Mass Spectrometry Laboratory. Analytical high performance liquid chromatography (HPLC) was performed on a HewlettPackard 1100 Series HPLC with a diode array detector using the indicated chiral column.

(i) Pangborn, A. B.; Giardello, M. A.; Grubbs, R. H.; Rosen, R. K.; Timmers, F. J. Organometallics 1996, 15, $1518-1520$.

(ii) Perrin, D. D. and Armarego, W. L. F. Purification of Laboratory Chemicals; $3^{\text {rd }}$ ed., Pergamon Press, Oxford, 1988. 


\section{Aluminum-Catalyzed Enantioselective Propargylsilane Additions.}

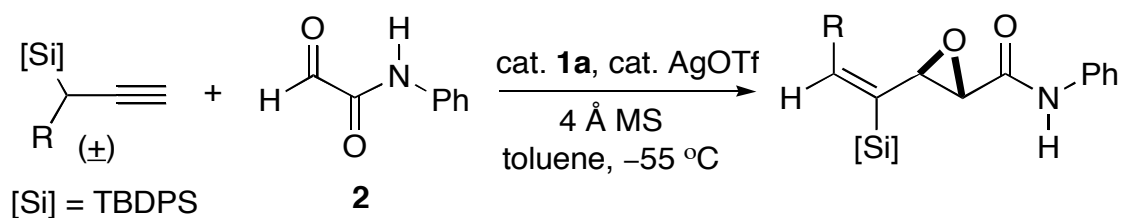

General Procedure A: ${ }^{\mathrm{iii}}$ To an oven-dried, 4-mL vial containing a magnetic stirring bar was added $N$ phenylglyoxamide 2 (14.9 mg, $0.1 \mathrm{mmol}),{ }^{\mathrm{iv}} \mathrm{Al}[(S)$-salbinam $] \mathrm{Cl} \mathbf{1 a}(7.8 \mathrm{mg}, 0.010 \mathrm{mmol}),{ }^{\mathrm{v}}$ AgOTf $(2.8$ $\mathrm{mg}, 0.011 \mathrm{mmol})$, and $4 \AA \mathrm{MS}(15.0 \mathrm{mg}, 150 \mathrm{mg} / \mathrm{mmol})$ in an inert atmosphere $\left(\mathrm{N}_{2}\right)$ glove box. [For Table 2, entry 1, $3.8 \mathrm{mg}, 0.011 \mathrm{mmol}$ of $\mathrm{AgSbF}_{6}$ was used instead]. The vial was fitted with a serum cap, wrapped in a tin foil (to minimize exposure to strong light) and removed from the inert atmosphere box. The vial was placed in an acetone/dry-ice bath $\left(-78^{\circ} \mathrm{C}\right)$ and was charged with toluene $(0.35 \mathrm{~mL})$. The resulting suspension was stirred at $-78{ }^{\circ} \mathrm{C}$ for $2 \mathrm{~min}$, followed by addition of the appropriate propargylsilane $(1.5 \text { equiv })^{\mathrm{vi}, \mathrm{vii}}$ via syringe at $-78^{\circ} \mathrm{C}$. The tin foil was removed and the vial was transferred to the cryocooler pre-set at $-55{ }^{\circ} \mathrm{C}$. The solution was stirred at that temperature over $30 \mathrm{~h}$ at which point the reaction was deemed complete by TLC. The reaction mixture was diluted by addition of pre-chilled 5\% EtOAc in Hexane $(3 \mathrm{~mL})$, filtered through an alumina (neutral Brockmann activity I) plug which was washed with $\mathrm{Et}_{2} \mathrm{O}$. The filtrate was concentrated in vacuo. The crude product was subjected to NMR and HPLC analyses, and may be purified by either flash chromatography (Method A) or recrystallization (Method B) as follows.

(iii) For Table 2, entries $3(\mathrm{R}=n$-Pr) and $4(\mathrm{R}=n$-Bu), reactions were conducted 20-times larger scales (i.e. 2.0 mmol scale of 2 ) and the corresponding amounts of reagents and solvents were scaled up appropriately.

(iv) For the synthesis of 2, see Evans, D. A.; Aye, Y.; Wu, J. Org. Lett. 2006, 8, 2071.

(v) For the synthesis of 1a, see Evans, D. A.; Janey, J. M.; Magomedov, N.; Tedrow, J. S. Angew. Chem. Int. Ed. 2001, 40, 1884.

(vi) Pre-prepared at room temperature as a $1 \mathrm{M}$ stock-solution in 3-F-toluene. Presence of 3-F-toluene (30\% by total solvent volume) was found to afford better reaction conversions, presumably due to increased solubility of glyoxamide 2 in a resulting more polar solvent system. However, running the same reactions either in neat 3-F-toluene or in a 1:1 mixture of toluene: 3-F-toluene gave lower ee's.

(vii) Unless otherwise indicated, the known propargylsilanes employed in this study were synthesized via isomerization of the corresponding allenes using $n$-BuLi (1.5 equiv) in $\mathrm{Et}_{2} \mathrm{O}$ at $\mathrm{rt}$ overnight followed by an aqueous workup and chromatographic purification over silica gel. For the syntheses of allenes, see (i) Evans, D. A.; Sweeney, Z. K.; Rovis, T.; Tedrow, J. S.; J. Am. Chem. Soc. 2001, 123, 12095; (ii) Danheiser, R. L.; Dixon, B. R.; Gleason, R. W. J. Org. Chem. 1992, 127, 8006. (Note: [Si] or TBDPS throughout this Supporting Information refers to tert-butyldiphenylsilyl, $t$-BuPh $\mathrm{Si}_{2}$ ). 
Purification method A: The crude product was loaded onto a neutral alumina (Brockmann activity I, no activation required) flash column and eluted with the indicated solvent to provide the title compounds. The excess silanes were recovered and reused without loss of selectivity.

Purification method B: The crude product was recrystallized from pentane $/ \mathrm{Et}_{2} \mathrm{O}\left(35^{\circ} \mathrm{C}\right.$ heating required to dissolve the pale yellow solid crude product) and allowed to stand overnight at room temperature to generate white crystalline solids.

\section{Scandium-catalyzed Non-enantioselective Reactions for the purpose of HPLC racemic assays.}

\section{General Procedure B [with (diiso-propylphenyl)propargylsilanes]:}

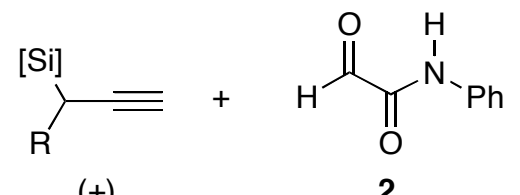

$( \pm)$

$[\mathrm{Si}]=i-\mathrm{Pr}_{2} \mathrm{PhSi}$

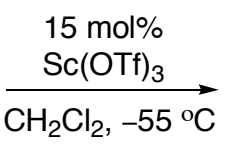

2

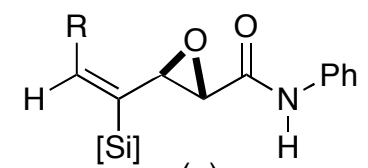

$( \pm)$

To an oven-dried, 4-mL vial containing a magnetic stirring bar was added 2 (14.9 mg, $0.1 \mathrm{mmol})$ and $\mathrm{Sc}(\mathrm{OTf})_{3}(7.2 \mathrm{mg}, 0.015 \mathrm{mmol})$ in an inert atmosphere $\left(\mathrm{N}_{2}\right)$ glove box. The vial was fitted with a serum cap and removed from the inert atmosphere box. The vial was charged with $\mathrm{CH}_{2} \mathrm{Cl}_{2}(0.7 \mathrm{~mL})$ at $\mathrm{rt}$ and cooled to $-55^{\circ} \mathrm{C}$. The appropriate (diisopropylphenyl)propargylsilane (1.5 equiv) was then added as a solution in $\mathrm{CH}_{2} \mathrm{Cl}_{2}(0.2 \mathrm{~mL})$ via syringe. The reaction was stirred at $-55{ }^{\circ} \mathrm{C}$ for $30 \mathrm{~min}$ after which time it was deemed complete by TLC. The reaction mixture was filtered through a silica gel plug which was washed with $\mathrm{Et}_{2} \mathrm{O}$. The filtrate was concentrated in vacuo. The crude product was then loaded onto a silica gel flash column and eluted with the indicated solvent to provide the corresponding racemic vinyl epoxides required for HPLC racemic assays. 


\section{General Procedure C [with (tert-butyldiphenyl)propargylsilanes]: ${ }^{\text {viii }}$}

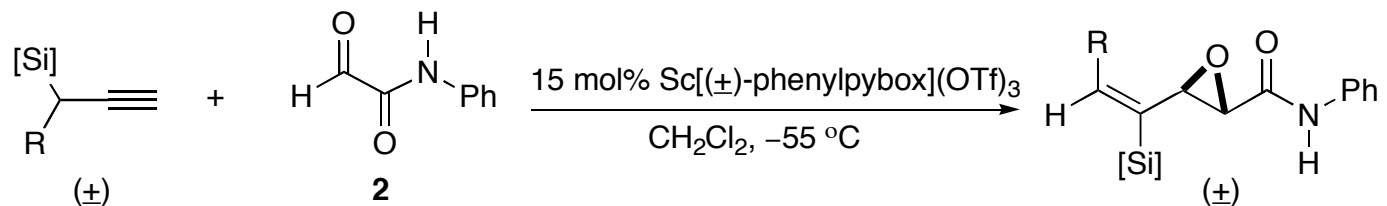
$[\mathrm{Si}]=\mathrm{TBDPS}$

To an oven-dried, 4-mL vial containing a magnetic stirring bar was added $(S, S)$-phenylpybox ${ }^{\mathrm{ix}}(2.5 \mathrm{mg}$, $0.008 \mathrm{mmol}),(R, R)$-phenylpybox $(2.5 \mathrm{mg}, 0.008 \mathrm{mmol}), \mathrm{Sc}(\mathrm{OTf})_{3}(7.2 \mathrm{mg}, 0.015 \mathrm{mmol})$, and $4 \AA \mathrm{MS}$ $(15.0 \mathrm{mg}, 150 \mathrm{mg} / \mathrm{mmol})$ in an inert atmosphere $\left(\mathrm{N}_{2}\right)$ glove box. To a separate vial was added 2 (14.9 $\mathrm{mg}, 0.1 \mathrm{mmol})$. Both vials were fitted with serum caps and removed from the inert atmosphere box. The vial containing the metal/ligand was charged with $\mathrm{CH}_{2} \mathrm{Cl}_{2}(0.7 \mathrm{~mL})$. The resulting suspension was stirred at room temperature for $1.5 \mathrm{~h}$. The vial was then transferred to the cryocooler pre-set at $-55^{\circ} \mathrm{C}$. To this vial was added $2(14.9 \mathrm{mg}, 0.1 \mathrm{mmol})$ as a solution in $\mathrm{CH}_{2} \mathrm{Cl}_{2}(0.3 \mathrm{~mL})$ via syringe. The vial that had contained 2 was rinsed with $\mathrm{CH}_{2} \mathrm{Cl}_{2}(0.2 \mathrm{~mL})$ and the solution was transferred to the reaction vial via syringe. This was followed by addition of the appropriate (tert-butyldiphenyl)propargylsilane (1.5 equiv) at $-55{ }^{\circ} \mathrm{C}$. The solution was stirred overnight at $-55^{\circ} \mathrm{C}$ at which point all reactions were deemed complete by TLC. The reaction mixture was filtered through a silica gel plug which was washed with $\mathrm{Et}_{2} \mathrm{O}$. The filtrate was concentrated in vacuo. The crude product was purified by either recrystallization from pentane $/ \mathrm{Et}_{2} \mathrm{O}$ or by flash column chromatography on silica gel to provide the corresponding racemic vinyl epoxides required for HPLC racemic assays.

(viii) With TBDPS-propargylsilanes, the corresponding non-enantioselective reactions in the absence of racemic ligands all led to decomposition.

(ix) During the course of this study, all pybox ligands were prepared as described in Davies, I. W.; Gerena, L.; Nu, L.; Larsen, R. D.; Reider, P. J. J. Org. Chem. 1996, 61, 9629. 


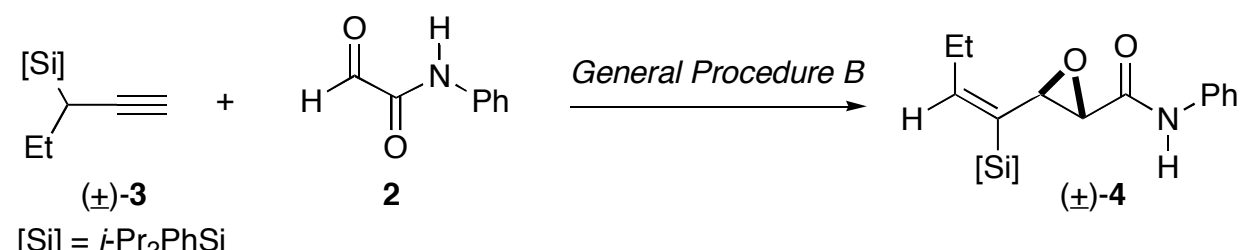

\section{$\{[( \pm), E]-3-(1-d i i s o-p r o p y l p h e n y l s i l y l) b u t-1-e n y l\}-N$-phenyloxirane-2-carboxamide $[( \pm)-4]$ :}

The indicated compound was prepared according to General Procedure B and isolated by flash chromatography (5\% EtOAc/hexanes) as a white crystalline solid that was homogeneous as judged by ${ }^{1}$ H NMR spectroscopy (85\% yield): mp 144-145 ${ }^{\circ} \mathrm{C}$; IR (film) 3287, 2951, 2863, 1659, 1601, 1545, 1443, 1105, 995, 900, 799, 758, 700, $662 \mathrm{~cm}^{-1}$; ${ }^{1} \mathrm{H}$ NMR (500 MHz, $\mathrm{CDCl}_{3}$ ) ठ 7.77 (brs, 1H), 7.54-7.49 (m, 3H), 7.39-7.26 (m, 6H), $7.12(\mathrm{t}, 1 \mathrm{H}, J=7.3 \mathrm{~Hz}), 6.07$ (t, 1H, $J=6.9 \mathrm{~Hz}), 3.77$ (brs, 1H), 3.35 (d, $1 \mathrm{H}, J=1.8 \mathrm{~Hz}), 2.36(\mathrm{o}, 2 \mathrm{H}, J=7.8 \mathrm{~Hz}), 1.47(\mathrm{~s}, 2 \mathrm{H}, J=7.8 \mathrm{~Hz}), 1.26-0.96(\mathrm{~m}, 9 \mathrm{H}) ;{ }^{13} \mathrm{C} \mathrm{NMR}(125$ $\left.\mathrm{MHz}, \mathrm{CDCl}_{3}\right) \delta 153.4,135.8,129.3,129.2,127.8,124.8,119.8,60.3,57.2,18.2,18.1,13.9,10.5$; HRMS (ESI): Exact mass calcd for $\mathrm{C}_{25} \mathrm{H}_{34} \mathrm{NO}_{2} \mathrm{Si}[\mathrm{M}+\mathrm{H}]^{+}$, requires $m / z: 408.2359$, found $m / z: 408.2360$.

The single crystal required for the X-ray analysis was grown from EtOAc/Hexanes using slow evaporation method. See attached crystal data tables and corresponding cif file (ya37.cif) for more information.

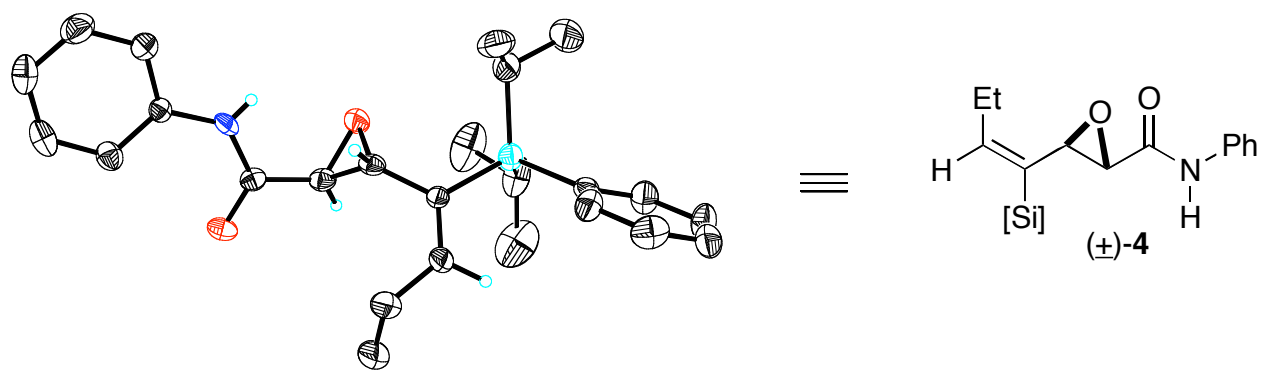




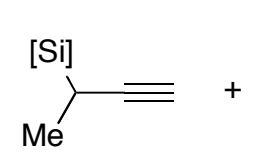

( \pm$)-8$

$[\mathrm{Si}]=\mathrm{TBDPS}$

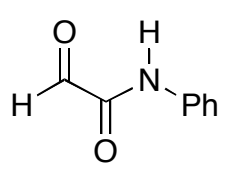

2
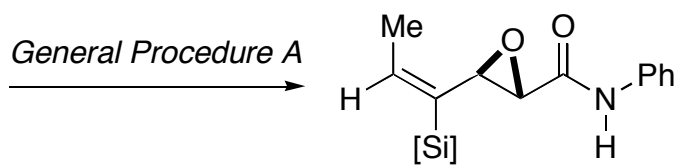

\section{(2S,3S,E)-3-(1-(tert-butyldiphenylsilyl)prop-1-enyl)- $N$-phenyloxirane-2-carboxamide:}

The indicated compound was prepared according to General Procedure A and isolated by flash chromatography on neutral alumina (gradient 2-5\% EtOAc/hexanes) as a white solid that was homogeneous as judged by ${ }^{1} \mathrm{H}$ NMR spectroscopy (86\% yield): mp $144-145{ }^{\circ} \mathrm{C} ;[\alpha]_{\mathrm{D}}-65.1 \quad(c=1.02$, $\mathrm{CHCl}_{3}$ ); IR (film) 3352, 3296, 3068, 2951, 2857, 1684, 1602, 1540, 1444, 1427, 1104, 1030, 890, 741 $\mathrm{cm}^{-1} ;{ }^{1} \mathrm{H}$ NMR $\left(500 \mathrm{MHz}, \mathrm{CDCl}_{3}\right) \delta 7.66$ (brs, $\left.1 \mathrm{H}\right), 7.60-7.58$ (m, 2H), 7.55-7.53 (m, 2H), $7.48(\mathrm{~d}, 2 \mathrm{H}, J$ $=7.3 \mathrm{~Hz}), 7.43-7.34(\mathrm{~m}, 6 \mathrm{H}), 7.30(\mathrm{t}, 2 \mathrm{H}, J=7.8 \mathrm{~Hz}), 7.10(\mathrm{t}, 1 \mathrm{H}, \mathrm{J}=7.3 \mathrm{~Hz}), 6.35(\mathrm{dq}, 1 \mathrm{H}, J=1.4 \mathrm{~Hz}$, $6.9 \mathrm{~Hz}), 3.79$ (brs, $1 \mathrm{H}), 3.30(\mathrm{~d}, 1 \mathrm{H}, J=2.3 \mathrm{~Hz}), 2.00(\mathrm{~d}, 3 \mathrm{H}, J=6.9 \mathrm{~Hz}), 1.17(\mathrm{~s}, 9 \mathrm{H}) ;{ }^{13} \mathrm{C}$ NMR $(125$ $\left.\mathrm{MHz}, \mathrm{CDCl}_{3}\right) \delta$ 166.4, 147.4, 137.0, 136.4, 136.3, 134.8, 132.0, 129.5, 129.3, 127.9, 124.8, 119.8, 60.4, 57.4, 29.1, 18.6, 16.9; HRMS (ESI): Exact mass calcd for $\mathrm{C}_{28} \mathrm{H}_{32} \mathrm{NO}_{2} \mathrm{Si}[\mathrm{M}+\mathrm{H}]^{+}$, requires $\mathrm{m} / z: 442.2202$, found $m / z$ : 442.2214; Enantiomeric excess determined by HPLC with Chiracel ADH column, $1 \% i$ PrOH/hexanes, $0.5 \mathrm{~mL} / \mathrm{min}, 254 \mathrm{~nm}$; $\mathrm{t}_{\mathrm{r}}($ minor $)=22.1, \mathrm{t}_{\mathrm{r}}($ major $)=25.3,92 \%$ ee.
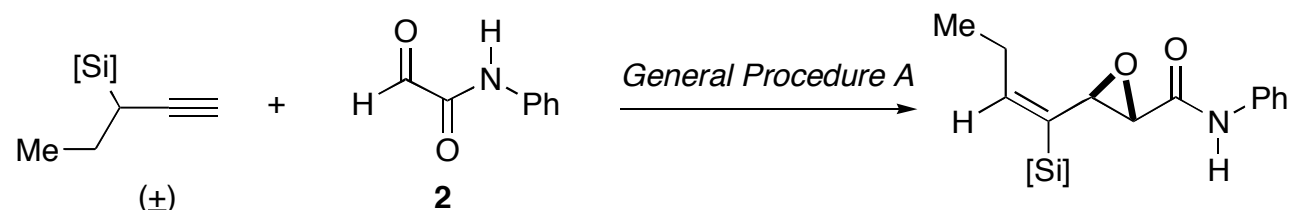

$[\mathrm{Si}]=\mathrm{TBDPS}$

\section{(2S,3S,E)-3-(1-(tert-butyldiphenylsilyl)but-1-enyl)- $N$-phenyloxirane-2-carboxamide:}

The indicated compound was prepared according to General Procedure A and isolated by flash chromatography on neutral alumina (gradient 2-5\% EtOAc/hexanes) as a white solid that was homogeneous as judged by ${ }^{1} \mathrm{H}$ NMR spectroscopy (70\% yield): $\mathrm{mp} 135-136{ }^{\circ} \mathrm{C} ;[\alpha]_{\mathrm{D}}-79.3 \quad(c=0.89$, $\mathrm{CHCl}_{3}$ ); IR (film) 3290, 3060, 2962, 2858, 1674, 1602, 1532, 1499, 1446, 1427, 1099, 904, 817, 756, $699 \mathrm{~cm}^{-1} ;{ }^{1} \mathrm{H}$ NMR $\left(500 \mathrm{MHz}, \mathrm{CDCl}_{3}\right) \delta 7.63$ (brs, 1H), 7.61-7.60 (m, 2H), 7.59-7.56 (m, 2H), 7.46 (d, $2 \mathrm{H}, J=7.8 \mathrm{~Hz}), 7.43-7.33(\mathrm{~m}, 6 \mathrm{H}), 7.30(\mathrm{t}, 2 \mathrm{H}, J=7.8 \mathrm{~Hz}), 7.10(\mathrm{t}, 1 \mathrm{H}, J=7.3 \mathrm{~Hz}), 6.26(\mathrm{dt}, 1 \mathrm{H}, J=$ $0.9 \mathrm{~Hz}, 7.8 \mathrm{~Hz}), 3.78$ (brs, 1H), 3.25 (d, 1H, $J=2.3 \mathrm{~Hz}), 2.42(\mathrm{o}, 2 \mathrm{H}, J=5.0 \mathrm{~Hz}), 1.17$ (s, 9H), 1.07 (t, 
$3 \mathrm{H}, J=7.8 \mathrm{~Hz}) ;{ }^{13} \mathrm{C} \mathrm{NMR}\left(125 \mathrm{MHz}, \mathrm{CDCl}_{3}\right) \delta 166.3,154.6,136.4,136.2,134.8,130.0,129.5,129.3$, 127.9, 124.8, 119.8, 60.3, 57.4, 29.1, 24.3, 18.7, 13.9; HRMS (ESI): Exact mass calcd for $\mathrm{C}_{29} \mathrm{H}_{34} \mathrm{NO}_{2} \mathrm{Si}$ $[\mathrm{M}+\mathrm{H}]^{+}$, requires $m / z: 456.2359$, found $m / z: 456.2375$; Enantiomeric excess determined by HPLC with Chiracel ADH column, 1\% $i$-PrOH/hexanes, $0.5 \mathrm{~mL} / \mathrm{min}, 254 \mathrm{~nm} ; \mathrm{t}_{\mathrm{r}}($ minor $)=28.1$, $\mathrm{t}_{\mathrm{r}}($ major $)=44.6$, $90 \%$ ee.
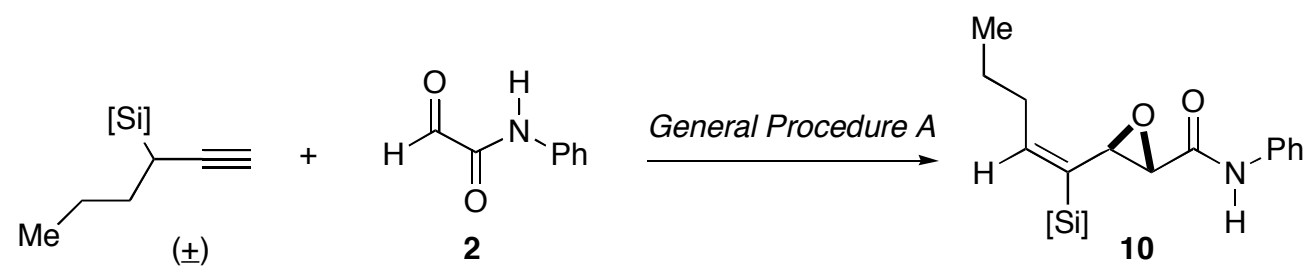

$[\mathrm{Si}]=\mathrm{TBDPS}$

\section{$(2 S, 3 S, E)-3-(1-(t e r t-b u t y l d i p h e n y l s i l y l) p e n t-1-e n y l)-N$-phenyloxirane-2-carboxamide (10):}

The indicated compound was prepared according to General Procedure A and isolated by either flash chromatography on neutral alumina (gradient 2-5\% EtOAc/hexanes) or re-crystallized from $\mathrm{Et}_{2} \mathrm{O} /$ Pentane as a white solid that was homogeneous as judged by ${ }^{1} \mathrm{H}$ NMR spectroscopy (77\% yield): mp 140-141 ${ }^{\circ} \mathrm{C} ;[\alpha]_{\mathrm{D}}-53.0\left(c=0.34, \mathrm{CHCl}_{3}\right)$; IR (film) 3291, 2960, 2858, 1684, 1653, 1601, 1558, 1532, 1444, 1428, 1104, $740 \mathrm{~cm}^{-1}$; ${ }^{1} \mathrm{H}$ NMR (500 MHz, $\mathrm{CDCl}_{3}$ ) $\delta 7.62$ (brs, 1H), 7.62-7.60 (m, 2H), 7.57-7.55 (m, 2H), $7.47(\mathrm{~d}, 2 \mathrm{H}, J=8.7 \mathrm{~Hz}), 7.42-7.25(\mathrm{~m}, 6 \mathrm{H}), 7.10(\mathrm{t}, 2 \mathrm{H}, J=7.3 \mathrm{~Hz}), 6.27(\mathrm{dt}, 1 \mathrm{H}, J=$ $1.4 \mathrm{~Hz}, 7.3 \mathrm{~Hz}$ ), 3.78 (brs, 1H), 3.24 (d, 1H, $J=2.4 \mathrm{~Hz}), 2.39$ (o, 2H, $J=5.0 \mathrm{~Hz}), 1.49$ (s, 2H, $J=12.4$ $\mathrm{Hz}), 1.17(\mathrm{~s}, 9 \mathrm{H}), 0.96$ (t, 3H, $J=7.3 \mathrm{~Hz}) ;{ }^{13} \mathrm{C} \mathrm{NMR}\left(125 \mathrm{MHz}, \mathrm{CDCl}_{3}\right) \delta 166.3,153.1,136.4,136.3$, $134.8,130.8,129.5,129.2,127.9,124.8,119.8,60.4,57.4,32.8,29.1,22.5,18.7$, 14.1; HRMS (ESI): Exact mass calcd for $\mathrm{C}_{30} \mathrm{H}_{36} \mathrm{NO}_{2} \mathrm{Si}[\mathrm{M}+\mathrm{H}]^{+}$, requires $m / z$ : 470.2515, found $m / z$ : 470.2495; Enantiomeric excess determined by HPLC with Chiracel ADH column, $1 \% i$-PrOH/hexanes, $0.5 \mathrm{~mL} / \mathrm{min}, 254 \mathrm{~nm}$; $\mathrm{t}_{\mathrm{r}}$ $($ minor $)=22.6, \mathrm{t}_{\mathrm{r}}($ major $)=36.8,94 \%$ ee. 
The single crystal for the X-ray analysis (obtained from racemic sample) was grown from EtOH/toluene mixture using slow evaporation method. See attached crystal data tables and corresponding cif file (ya59sad.cif) for more information.

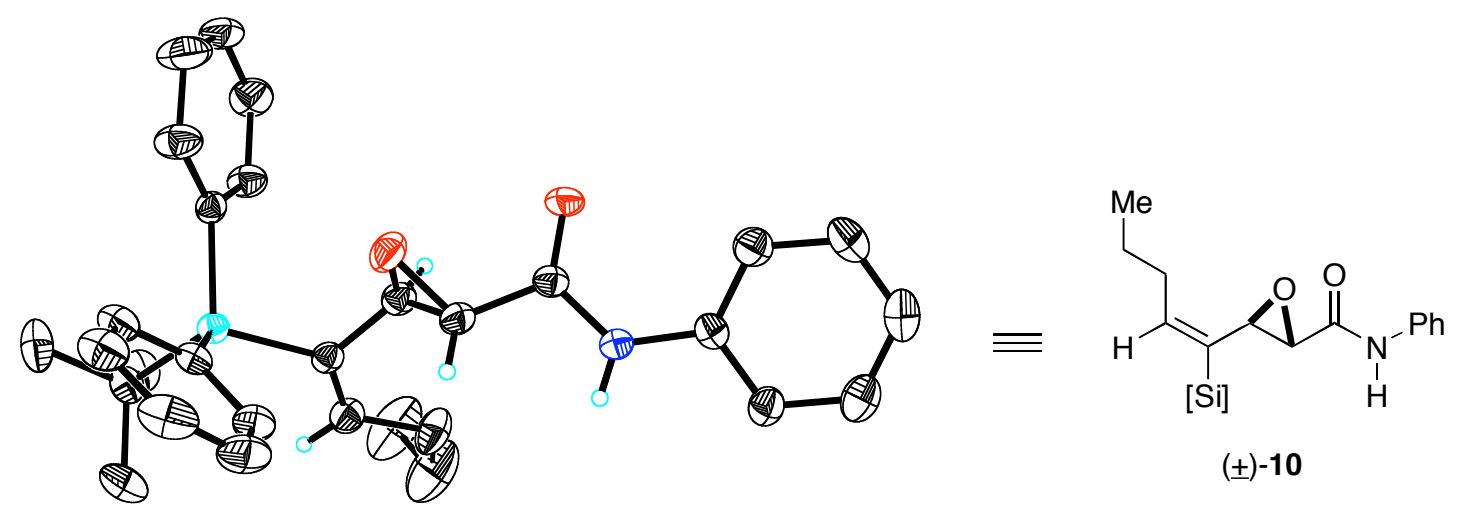

Ortep Diagram for Single Crystal X-ray Structure

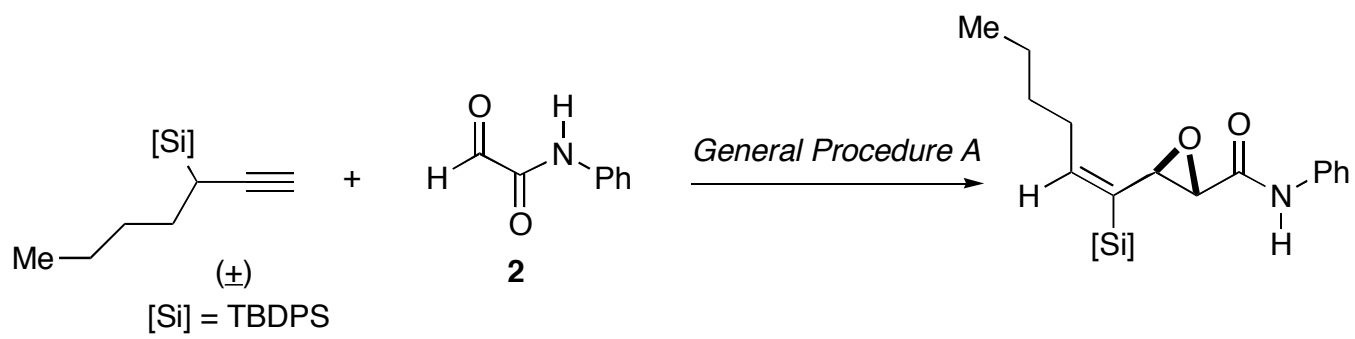

\section{(2S,3S,E)-3-(1-(tert-butyldiphenylsilyl)hex-1-enyl)- $N$-phenyloxirane-2-carboxamide:}

The indicated compound was prepared according to General Procedure A and isolated by either flash chromatography on neutral alumina (gradient 2-5\% EtOAc/hexanes) or re-crystallized from $\mathrm{Et}_{2} \mathrm{O} /$ Pentane as a white solid that was homogeneous as judged by ${ }^{1} \mathrm{H}$ NMR spectroscopy (97\% yield):

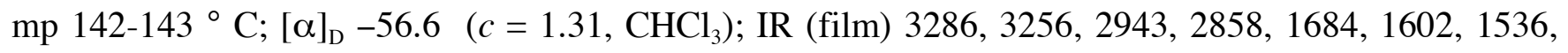
1445, 1427, 1104, 901, 819, 740, $701 \mathrm{~cm}^{-1} ;{ }^{1} \mathrm{H}$ NMR (500 MHz, CDCl ${ }_{3}$ ) 7.63 (brs, 1H), 7.62-7.61 (m, 2H), 7.57-7.56 (m, 2H), $7.47(\mathrm{~d}, 2 \mathrm{H}, J=7.3 \mathrm{~Hz}), 7.43-7.34(\mathrm{~m}, 6 \mathrm{H}), 7.30(\mathrm{t}, 2 \mathrm{H}, J=8.3 \mathrm{~Hz}), 7.10(\mathrm{t}$, 
$1 \mathrm{H}, J=7.8 \mathrm{~Hz}$ ), $6.28(\mathrm{dt}, 1 \mathrm{H}, J=1.4 \mathrm{~Hz}, 7.3 \mathrm{~Hz}), 3.78$ (brs, 1H), 3.25 (d, 1H, $J=2.3 \mathrm{~Hz}), 2.39(\mathrm{~m}, 2 \mathrm{H})$, 1.47-1.36 (m, 4H), $1.17(\mathrm{~s}, 9 \mathrm{H}), 0.94(\mathrm{t}, 3 \mathrm{H}, J=7.3 \mathrm{~Hz}) ;{ }^{13} \mathrm{C} \mathrm{NMR}\left(125 \mathrm{MHz}, \mathrm{CDCl}_{3}\right) \delta 166.3,153.3$, $137.1,136.4,136.3,134.8,130.5,129.5,129.2$, 127.9, 124.8, 119.8, 60.4, 57.4, 31.5, 30.7, 29.1, 22.7, 18.7, 14.2; HRMS (ESI): Exact mass calcd for $\mathrm{C}_{31} \mathrm{H}_{38} \mathrm{NO}_{2} \mathrm{Si}[\mathrm{M}+\mathrm{H}]^{+}$, requires $\mathrm{m} / z$ : 484.2672, found $\mathrm{m} / z$ : 484.2672; Enantiomeric excess determined by HPLC with Chiracel ADH column, 1\% $i$-PrOH/hexanes, $0.5 \mathrm{~mL} / \mathrm{min}, 254 \mathrm{~nm} ; \mathrm{t}_{\mathrm{r}}($ minor $)=19.9, \mathrm{t}_{\mathrm{r}}($ major $)=38.6,92 \%$ ee.

The single crystal for the X-ray analysis (obtained from racemic sample) was grown from $\mathrm{CH}_{2} \mathrm{Cl}_{2}$ /heptane mixture using slow evaporation method. See attached crystal data tables and corresponding cif file (ya58sad.cif) for more information.
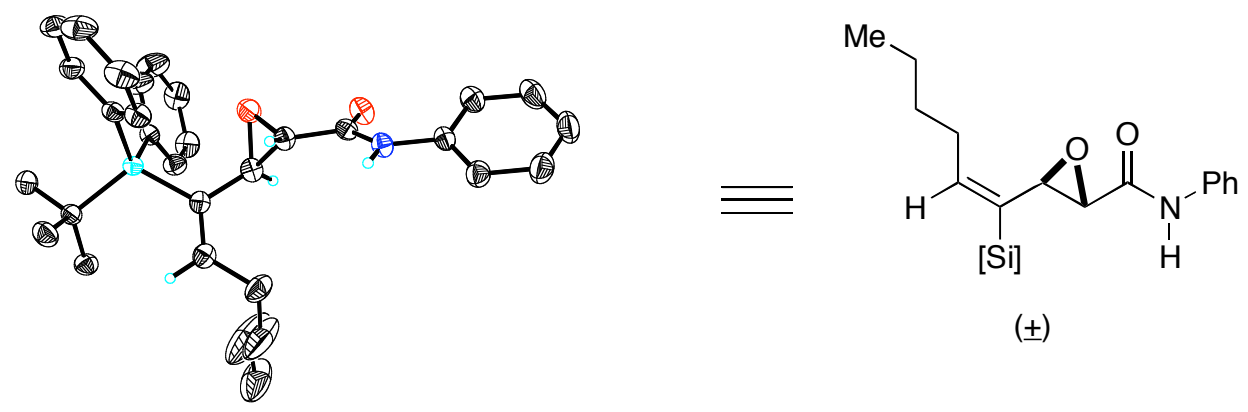

$( \pm)$

Ortep Diagram for Single Crystal X-ray Structure

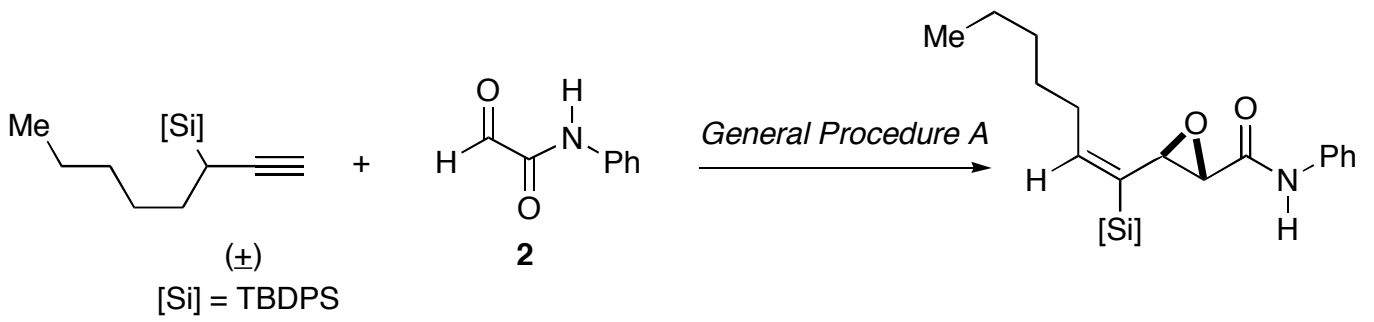

\section{(2S,3S,E)-3-(1-(tert-butyldiphenylsilyl)hept-1-enyl)- $N$-phenyloxirane-2-carboxamide:}

The indicated compound was prepared according to General Procedure A and isolated by flash chromatography on neutral alumina (gradient 2-5\% EtOAc/hexanes) as a white solid that was homogeneous as judged by ${ }^{1} \mathrm{H}$ NMR spectroscopy (73\% yield): mp $117-118^{\circ} \mathrm{C}$; $[\alpha]_{\mathrm{D}}-33.0 \quad(c=1.45$, $\mathrm{CHCl}_{3}$ ); IR (film) 3294, 3238, 2943, 2857, 1684, 1602, 1539, 1445, 1427, 1104, 889, 819, 741, 701 cm 
${ }^{1} \mathrm{H}$ NMR $\left(500 \mathrm{MHz}, \mathrm{CDCl}_{3}\right) \delta 7.64$ (brs, 1H), 7.63-7.61 (m, 2H), 7.58-7.56 (m, 2H), 7.47 (d, 2H, J= 7.3 $\mathrm{Hz}), 7.43-7.34(\mathrm{~m}, 6 \mathrm{H}), 7.30(\mathrm{t}, 2 \mathrm{H}, J=8.3 \mathrm{~Hz}), 7.11(\mathrm{t}, 1 \mathrm{H}, J=7.8 \mathrm{~Hz}), 6.28(\mathrm{dt}, 1 \mathrm{H}, J=1.5 \mathrm{~Hz}, 7.1$ Hz), 3.78 (brs, 1H), 3.25 (d, 1H, $J=2.4 \mathrm{~Hz}), 2.44-2.39$ (m, 2H), 1.48-1.45 (m, 2H), 1.36-1.32 (m, 4H), $1.18(\mathrm{~s}, 9 \mathrm{H}), 0.91(\mathrm{t}, 3 \mathrm{H}, J=6.8 \mathrm{~Hz}) ;{ }^{13} \mathrm{C} \mathrm{NMR}\left(125 \mathrm{MHz}, \mathrm{CDCl}_{3}\right) \delta 166.3,153.4,137.1,136.4,136.3$, $134.8,129.5,129.3,127.9,124.8,119.8,60.4,57.4,31.8,30.9,29.1,29.0,22.8,18.7,14.3$; HRMS (ESI): Exact mass calcd for $\mathrm{C}_{32} \mathrm{H}_{40} \mathrm{NO}_{2} \mathrm{Si}[\mathrm{M}+\mathrm{H}]^{+}$, requires $m / z$ : 498.2828, found $m / z$ : 498.2829; Enantiomeric excess determined by HPLC with Chiracel ADH column, $1 \% i$-PrOH/hexanes, 0.5 $\mathrm{mL} / \min , 254 \mathrm{~nm} ; \mathrm{t}_{\mathrm{r}}($ minor $)=19.3, \mathrm{t}_{\mathrm{r}}($ major $)=33.1,90 \%$ ee.

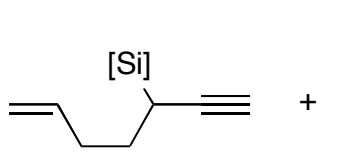

$( \pm)$

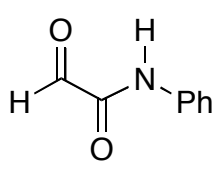

2

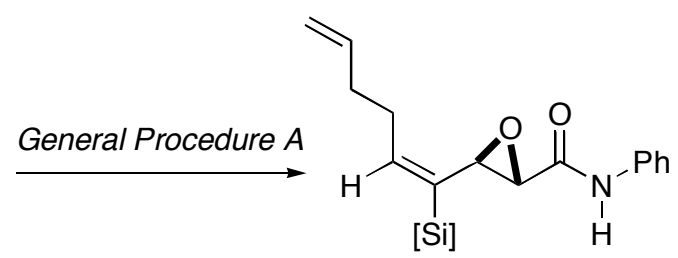

$[\mathrm{Si}]=\mathrm{TBDPS}$

\section{(2S,3S,E)-3-(1-(tert-butyldiphenylsilyl)hexa-1,5-dienyl)- $N$-phenyloxirane-2-carboxamide: ${ }^{\mathrm{x}}$}

The indicated compound was prepared according to General Procedure A and isolated by flash chromatography on neutral alumina (gradient 5-10\% EtOAc/hexanes) as a white solid that was homogeneous as judged by ${ }^{1} \mathrm{H}$ NMR spectroscopy (49\% yield): mp $131-132{ }^{\circ} \mathrm{C} ;[\alpha]_{\mathrm{D}}-8.88(c=0.12$, $\mathrm{CHCl}_{3}$ ); IR (film) 3294, 3100, 2983, 2844, 1684, 1639, 1602, 1534, 1454, 1430, 1100, 900, 879, 741, $704 \mathrm{~cm}^{-1} ;{ }^{1} \mathrm{H}$ NMR (400 MHz, $\left.\mathrm{CDCl}_{3}\right) \delta 7.62$-7.54 (m, 5H), 7.46 (d, 2H, J = 7.7 Hz), 7.44-7.26 (m, 8H), $7.10(\mathrm{t}, 2 \mathrm{H}, J=7.3 \mathrm{~Hz}), 6.26(\mathrm{t}, 1 \mathrm{H}, J=7.3 \mathrm{~Hz}), 5.86-5.18(\mathrm{~m}, 1 \mathrm{H}), 5.07-5.01(\mathrm{~m}, 2 \mathrm{H}), 3.77(\mathrm{brs}, 1 \mathrm{H})$, $3.24(\mathrm{~d}, 1 \mathrm{H}, J=1.8 \mathrm{~Hz}), 2.52(\mathrm{q}, 2 \mathrm{H}, J=7.3 \mathrm{~Hz}), 2.22(\mathrm{q}, 2 \mathrm{H}, J=7.0 \mathrm{~Hz}), 1.16(\mathrm{~s}, 9 \mathrm{H}) ;{ }^{13} \mathrm{C}$ NMR $(100$ $\left.\mathrm{MHz}, \mathrm{CDCl}_{3}\right) \delta 166.3,152.0,137.8,136.4,136.3,134.7,131.2,129.5,129.3,128.0,124.8,119.8$, 115.9, 60.4, 57.3, 33.4, 30.1, 29.0, 18.6; HRMS (ESI): Exact mass calcd for $\mathrm{C}_{31} \mathrm{H}_{36} \mathrm{NO}_{2} \mathrm{Si}[\mathrm{M}+\mathrm{H}]^{+}$, requires $m / z: 482.2515$, found $m / z: 482.2505$; Enantiomeric excess determined by HPLC with Chiracel $\mathrm{ADH}$ column, 1\% $i$-PrOH/hexanes, $0.5 \mathrm{~mL} / \mathrm{min}, 254 \mathrm{~nm}$; $\mathrm{t}_{\mathrm{r}}($ minor $)=25.7$, $\mathrm{t}_{\mathrm{r}}$ (major) = 45.2, 94\% ee.

\footnotetext{
(x) We thank Dr. Zachary K. Sweeney and Prof. Tomislav Rovis for leaving behind a stock of corresponding allenes, precursors to the indicated propargylsilanes. The allenes were prepared via $\mathrm{Zn}$ insertion to either 5-iodo-2-methylpent-2-ene or 4-iodobut-1-ene, followed by cuprate-mediated conjugate additions of resulting organozincates to the mesylate derivative of prop-2-yn-1-ol according to the analogous procedure described in Evans, D. A.; Sweeney, Z. K.; Rovis, T.; Tedrow, J. S.; J. Am. Chem. Soc. 2001, 123, 12095.
} 


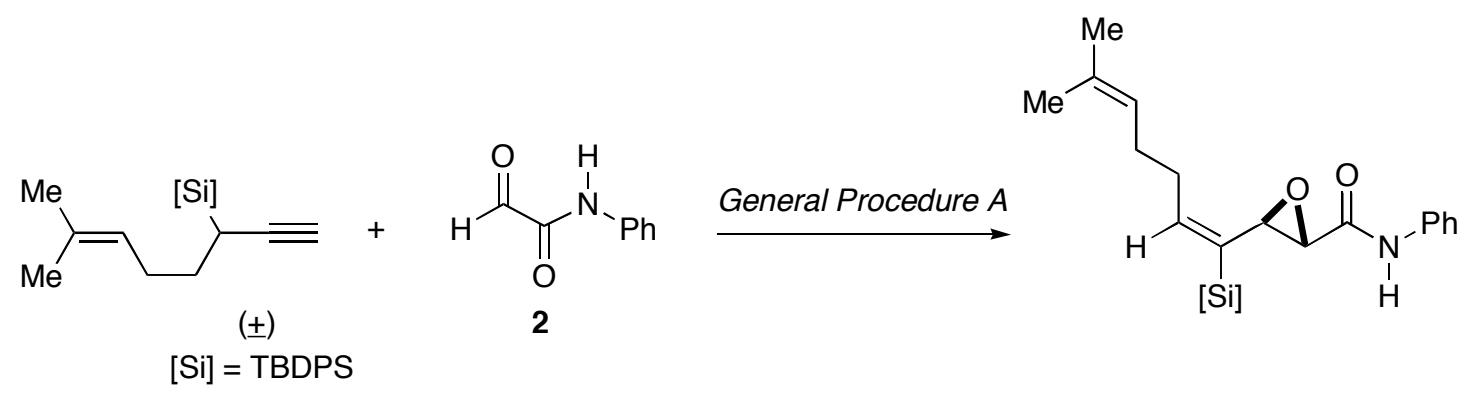

\section{(2S,3S,E)-3-(1-(tert-butyldiphenylsilyl)-6-methylhepta-1,5-dienyl)- $N$-phenyloxirane-2-} carboxamide: viii

The indicated compound was prepared according to General Procedure A and isolated by flash chromatography on neutral alumina (gradient 5-10\% EtOAc/hexanes) as a white solid that was homogeneous as judged by ${ }^{1} \mathrm{H}$ NMR spectroscopy (83\% yield): $\mathrm{mp} 104-105{ }^{\circ} \mathrm{C} ;[\alpha]_{\mathrm{D}}-28.0 \quad(c=0.97$, $\mathrm{CHCl}_{3}$ ); IR (film) 3320, 3297, 2998, 2858, 1688, 1602, 1630, 1444, 1427, 1104, 1032, 905, 820, $740 \mathrm{~cm}$ ${ }^{1}$; ${ }^{1} \mathrm{H}$ NMR (500 MHz, $\mathrm{CDCl}_{3}$ ) $\delta 7.63$ (brs, 1H), 7.62 -7.59 (m, 2H), 7.56-7.54 (m, 2H), 7.48-7.46 (m, 2H), 7.42-7.28 (m, 8H), 7.10 (t, 1H, $J=7.3 \mathrm{~Hz}), 6.24(\mathrm{dt}, 1 \mathrm{H}, J=1.4 \mathrm{~Hz}, 7.3 \mathrm{~Hz}), 5.13(\mathrm{tt}, 1 \mathrm{H}, J=1.4$ $\mathrm{Hz}, 7.3 \mathrm{~Hz}), 3.76(\mathrm{~d}, 1 \mathrm{H}, J=0.9 \mathrm{~Hz}), 3.24(\mathrm{~d}, 1 \mathrm{H}, J=2.3 \mathrm{~Hz}), 2.49-2.44(\mathrm{~m}, 2 \mathrm{H}), 2.16(\mathrm{q}, 2 \mathrm{H}, J=6.9$ $\mathrm{Hz}), 1.69(\mathrm{~d}, 3 \mathrm{H}, J=0.9 \mathrm{~Hz}), 1.56$ (brs, 3H), $1.16(\mathrm{~s}, 9 \mathrm{H}) ;{ }^{13} \mathrm{C} \mathrm{NMR}\left(125 \mathrm{MHz}, \mathrm{CDCl}_{3}\right) \delta 166.3,152.7$, 137.1, 136.4, 136.3, 134.8, 132.8, 130.8, 129.5, 129.2, 127.9, 124.8, 123.7, 119.8, 60.4, 57.4, 30.9, 29.0, 27.6, 25.9, 18.6, 17.9; HRMS (ESI): Exact mass calcd for $\mathrm{C}_{33} \mathrm{H}_{40} \mathrm{NO}_{2} \mathrm{Si}[\mathrm{M}+\mathrm{H}]^{+}$, requires $m / z: 510.2828$, found $m / z$ : 510.2837; Enantiomeric excess determined by HPLC with Chiracel ADH column, $1 \% i$ $\mathrm{PrOH} / \mathrm{hexanes}, 0.5 \mathrm{~mL} / \mathrm{min}, 254 \mathrm{~nm}$; $\mathrm{t}_{\mathrm{r}}($ minor $)=20.4, \mathrm{t}_{\mathrm{r}}$ (major) $=30.9,85 \%$ ee. 


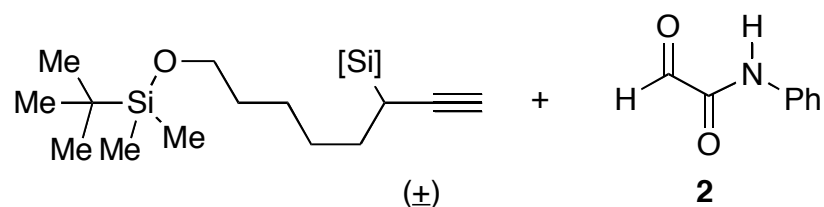

$[\mathrm{Si}]=\mathrm{TBDPS}$

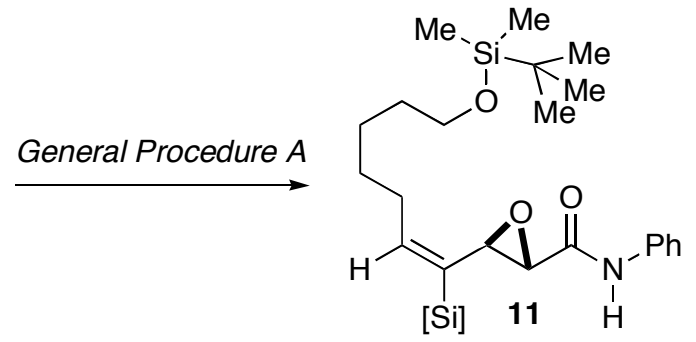

(2S,3S,E)-3-(7-(tert-butyldimethylsilyloxy)-1-(tert-butyldiphenylsilyl)hept-1-enyl)- $N$-phenyloxirane2-carboxamide (11): $:^{\mathrm{xi}}$

The indicated compound was prepared according to General Procedure A and isolated by flash chromatography on neutral alumina (gradient 2-5\% EtOAc/hexanes) as a colorless oil that was homogeneous as judged by ${ }^{1} \mathrm{H}$ NMR spectroscopy (76\% yield): $[\alpha]_{\mathrm{D}}-39.4$ ( $\left.c=1.07, \mathrm{CHCl}_{3}\right)$; IR (film) 3327, 2929, 2857, 1684, 1602, 1530, 1444, 1427, 1255, 1103, $894 \mathrm{~cm}^{-1} ;{ }^{1} \mathrm{H}$ NMR (500 MHz, $\left.\mathrm{CDCl}_{3}\right) \delta$ 7.63 (brs, 1H), $7.61-7.60(\mathrm{~m}, 2 \mathrm{H}), 7.57-7.55(\mathrm{~m}, 2 \mathrm{H}), 7.48-7.46(\mathrm{~m}, 2 \mathrm{H}), 7.42-7.33(\mathrm{~m}, 6 \mathrm{H}), 7.30(\mathrm{t}, 2 \mathrm{H}$, $J=7.3 \mathrm{~Hz}), 7.10(\mathrm{t}, 1 \mathrm{H}, J=7.8 \mathrm{~Hz}), 6.27(\mathrm{dt}, 1 \mathrm{H}, J=1.0 \mathrm{~Hz}, 6.3 \mathrm{~Hz}), 3.77(\mathrm{brs}, 1 \mathrm{H}), 3.61(\mathrm{t}, 2 \mathrm{H}, J=$ $6.8 \mathrm{~Hz}), 3.23(\mathrm{~d}, 1 \mathrm{H}, J=1.9 \mathrm{~Hz}), 2.42$, (q, 2H, $J=6.8 \mathrm{~Hz}), 1.56-1.37(\mathrm{~m}, 6 \mathrm{H}), 1.17(\mathrm{~s}, 9 \mathrm{H}), 0.89(\mathrm{~s}, 9 \mathrm{H})$, $0.04(\mathrm{~s}, 6 \mathrm{H}) ;{ }^{13} \mathrm{C}$ NMR $\left(125 \mathrm{MHz}, \mathrm{CDCl}_{3}\right) \delta 166.3,153.1,137.1,136.4,136.3,134.8,130.6,129.5$, 129.2, 127.9, 124.8, 119.8, 63., 60.3, 57.4, 32.9, 30.9, 29.2, 29.1, 26.2, 25.9, 18.7, 18.6, -5.0; HRMS (ESI): Exact mass calcd for $\mathrm{C}_{38} \mathrm{H}_{54} \mathrm{NO}_{3} \mathrm{Si}[\mathrm{M}+\mathrm{H}]^{+}$, requires $\mathrm{m} / z$ : 628.3642, found $\mathrm{m} / z$ : 628.3633; Enantiomeric excess determined by HPLC with Chiracel ADH column, $1 \%$-PrOH/hexanes, 0.5 $\mathrm{mL} / \mathrm{min}, 254 \mathrm{~nm} ; \mathrm{t}_{\mathrm{r}}($ minor $)=12.6, \mathrm{t}_{\mathrm{r}}($ major $)=18.4,91 \%$ ee .

(xi) The corresponding allene, precursor to the indicated known propargylsilane was synthesized via $\mathrm{Zn}$ insertion to TBSprotected 5-iodopentan-1-ol followed by cuprated-mediated conjugate addition of resulting organozincate to the mesylate derivative of prop-2-yn-1-ol according to the procedure described in Evans, D. A.; Sweeney, Z. K.; Rovis, T.; Tedrow, J. S.; $J$. Am. Chem. Soc. 2001, 123, 12095. TBS-protected 5-iodopentan-1-ol was prepared in 2-step from 5-TBShydroxypentanal $\left(\mathrm{NaBH}_{4}\right.$ reduction followed by Appel reaction). Mr. Eugene Kwan is acknowledged for providing the precursor 5TBShydroxypentanal. 


\section{Transformation of Vinyl Epoxide 10 to Allenic Alcohol 12.}

$[2 S,(3,4,5 S)]-2$-hydroxy- $N$-phenylocta-3,4-dienamide (12):

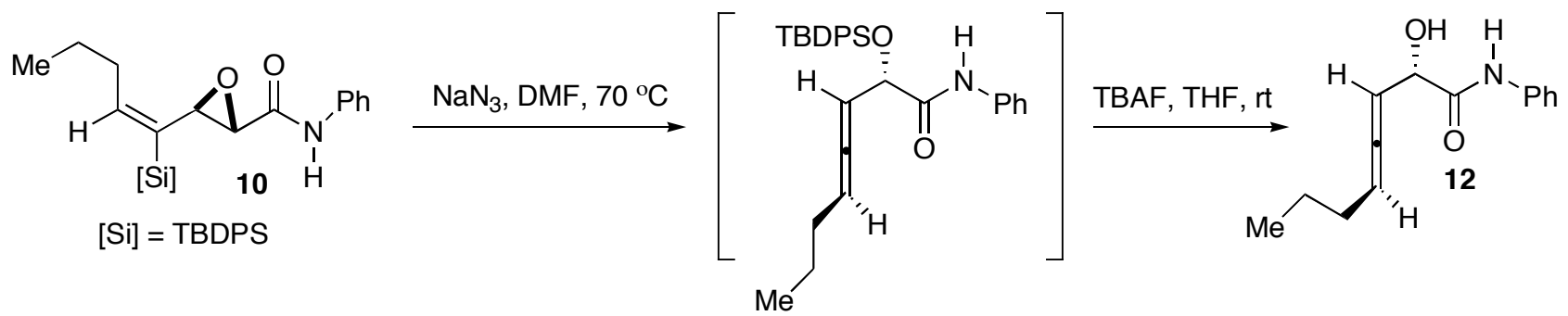

A 4-dram vial with a magnetic stirrer bar containing vinyl epoxide $10(46.9 \mathrm{mg}, 0.1 \mathrm{mmol})$ and $\mathrm{NaN}_{3}$ (19.5 mg, $0.3 \mathrm{mmol})$ was charged with DMF $(0.1 \mathrm{~mL})$ at room temperature. ${ }^{\mathrm{xii}}$ The vial was then transferred to a pre-heated oil bath at $70{ }^{\circ} \mathrm{C}$ and the stirred reaction mixture was heated for $30 \mathrm{~h}$. The reaction mixture was then filtered through a silica gel plug and washed with $\mathrm{Et}_{2} \mathrm{O}$. After evaporation under reduced pressure, the crude residue was re-dissolved in THF $(2.0 \mathrm{~mL})$ and charged with TBAF. $2 \mathrm{H}_{2} \mathrm{O}$ (158 mg, $\left.0.5 \mathrm{mmol}\right)$. The mixture was stirred at room temperature for $30 \mathrm{~min}$ and was diluted with $\mathrm{Et}_{2} \mathrm{O}$, washed with $\mathrm{H}_{2} \mathrm{O}$. The aqueous layer was back extracted with $\mathrm{Et}_{2} \mathrm{O}$ (x2). The combined organic layers were dried over $\mathrm{MgSO}_{4}$ and concentrated in vacuo. The crude residue was purified by silica gel flash column chromatography (15\% EtOAc/Hexanes) to give allenic alcohol $\mathbf{1 2}$ as a white crystalline solid that was homogeneous as judged by ${ }^{1} \mathrm{H}$ NMR spectroscopy (78\% yield): $\mathrm{mp} 80$ $81^{\circ} \mathrm{C} ;[\alpha]_{\mathrm{D}}-146\left(c=0.50, \mathrm{CHCl}_{3}\right)$; IR (film) 3374, 2924, 1664, 1600, 1534, 1444, 1318, 1261, 1066, $750 \mathrm{~cm}^{-1} ;{ }^{1} \mathrm{H}$ NMR $\left(600 \mathrm{MHz}, \mathrm{C}_{6} \mathrm{D}_{6}\right) \delta 7.95$ (brs, $\left.1 \mathrm{H}\right), 7.51$ (d, $\left.1 \mathrm{H}, J=8.2 \mathrm{~Hz}\right), 7.49$ (d, $\left.1 \mathrm{H}, J=8.3 \mathrm{~Hz}\right)$, $7.03(\mathrm{q}, 2 \mathrm{H}, J=7.6 \mathrm{~Hz}), 6.85-6.81(\mathrm{~m}, 1 \mathrm{H}), 5.55-5.52(\mathrm{~m}, 1 \mathrm{H}), 5.17-5.13(\mathrm{~m}, 1 \mathrm{H}), 4.46-4.42(\mathrm{~m}, 1 \mathrm{H})$, $2.47(\mathrm{~d}, 1 \mathrm{H}, J=4.5 \mathrm{~Hz}), 1.78-1.72(\mathrm{~m}, 2 \mathrm{H}), 1.25-1.19(\mathrm{~m}, 2 \mathrm{H}), 0.77-0.48(\mathrm{~m}, 3 \mathrm{H}) ;{ }^{13} \mathrm{C} \mathrm{NMR}(125 \mathrm{MHz}$, $\left.\mathrm{C}_{6} \mathrm{D}_{6}\right) \delta 202.6,169.2,138.1,129.1,127.9,124.2,119.5,96.3,92.9,70.2,30.6,22.3,13.6,13.5$; HRMS (ESI): Exact mass calcd for $\mathrm{C}_{14} \mathrm{H}_{18} \mathrm{NO}_{2}[\mathrm{M}+\mathrm{H}]^{+}$, requires $m / z: 232.1337$, found $m / z: 232.1331$; Enantiomeric excess determined by HPLC with Chiracel OJH column, 20\% $i$-PrOH/hexanes, 1.0 $\mathrm{mL} / \min , 254 \mathrm{~nm} ; \mathrm{t}_{\mathrm{r}}($ minor $)=4.65, \mathrm{t}_{\mathrm{r}}($ major $)=5.10,94 \%$ ee .

The intermediate $O$-TBDPS protected allenic alcohol may also be isolated and purified by flash column chromatography on silica gel as a pale yellow oil that was homogeneous as judged by ${ }^{1} \mathrm{H}$ NMR spectroscopy (80\% yield): $[\alpha]_{\mathrm{D}}-14.7\left(c=1.07, \mathrm{CHCl}_{3}\right.$ ); IR (film) 3354, 2974, 1668, 1600, 1529, 1400, 1335, 1259, 1060, 1057, $760 \mathrm{~cm}^{-1}$; ${ }^{1} \mathrm{H}$ NMR (500 MHz, $\left.\mathrm{C}_{6} \mathrm{D}_{6}\right) \delta 8.58$ (brs, $\left.1 \mathrm{H}\right), 7.68$ (dt, $5 \mathrm{H}, J=1.0 \mathrm{~Hz}$,

(xii) 12 was also isolated as a major diastereomer (97:3 dr) in an analogous reaction with NaSCN (24.3 mg, $0.3 \mathrm{mmol})$. 
$8.8 \mathrm{~Hz}), 7.61(\mathrm{dd}, 2 \mathrm{H}, J=3.9 \mathrm{~Hz}, 7.8 \mathrm{~Hz}), 7.20-7.10(\mathrm{~m}, 2 \mathrm{H}), 7.09-7.03(\mathrm{~m}, 5 \mathrm{H}), 6.83(\mathrm{t}, 1 \mathrm{H}, J=7.8$ $\mathrm{Hz}), 5.42(\mathrm{~h}, 1 \mathrm{H}, J=2.9 \mathrm{~Hz}), 5.08(\mathrm{dq}, 1 \mathrm{H}, J=2.4 \mathrm{~Hz}, 6.6 \mathrm{~Hz}), 4.96(\mathrm{dd}, 1 \mathrm{H}, J=2.4 \mathrm{~Hz}, 3.2 \mathrm{~Hz}), 1.71$ $(\mathrm{dq}, 2 \mathrm{H}, J=2.93 \mathrm{~Hz}, 7.6 \mathrm{~Hz}), 1.23-1.15(\mathrm{~m}, 2 \mathrm{H}), 0.72(\mathrm{t}, 3 \mathrm{H}, J=7.3 \mathrm{~Hz}) ;{ }^{13} \mathrm{C} \mathrm{NMR}\left(125 \mathrm{MHz}, \mathrm{C}_{6} \mathrm{D}_{6}\right) \delta$ 204.6, 168.8, 138.4, 136.0, 135.8, 130.4, 129.2, 124.1, 119.2, 94.8, 92.2, 73.9, 30.6, 27.0, 22.4, 19.4, 13.7; HRMS (ESI): Exact mass calcd for $\mathrm{C}_{30} \mathrm{H}_{36} \mathrm{NO}_{2} \mathrm{Si}[\mathrm{M}+\mathrm{H}]^{+}$, requires $\mathrm{m} / z$ : 470.2515 , found $\mathrm{m} / z$ : 470.2524 .

\section{Ag(I)-mediated Stereospecific Cyclization of Allenic Alcohol 12. Determination of Allene Geometry. ${ }^{\text {xiii }}$}
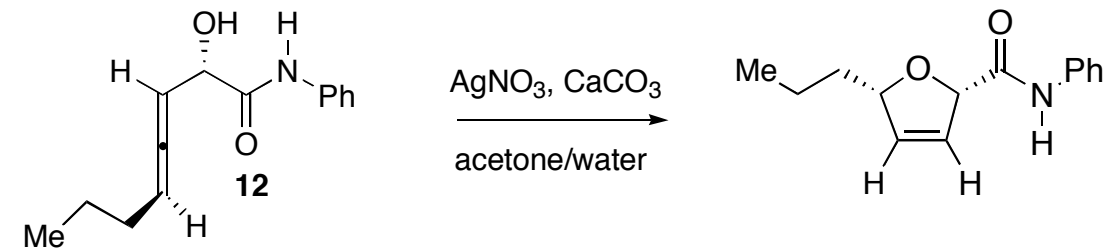

Allenic alcohol $12(19.6 \mathrm{mg}, 0.085 \mathrm{mmol})$ was dissolved in acetone $(0.6 \mathrm{~mL})$ and $\mathrm{H}_{2} \mathrm{O}(0.2 \mathrm{~mL})$ in a foil-wrapped round-bottom flask. $\mathrm{AgNO}_{3}(14.4 \mathrm{mg}, 0.085 \mathrm{mmol})$ and $\mathrm{CaCO}_{3}(8.49 \mathrm{mg}, 0.085 \mathrm{mmol})$ were added and the mixture was stirred at rt overnight. The solids were removed by filtration through a silica gel plug and the residue was concentrated under reduced pressure. The mixture was diluted with $\mathrm{Et}_{2} \mathrm{O}$ and $\mathrm{H}_{2} \mathrm{O}$; back extracted with $\mathrm{Et}_{2} \mathrm{O}(\mathrm{x} 2)$; dried over $\mathrm{Na}_{2} \mathrm{SO}_{4}$; concentrated under reduced pressure; and chromatographed on silica to afford the indicated dihydropyran above as a white glassy solid that was homogeneous as judged by ${ }^{1} \mathrm{H}$ NMR spectroscopy $\left(95 \%\right.$ yield): mp 88-89 ${ }^{\circ} \mathrm{C}$; $[\alpha]_{\mathrm{D}}-207(c=0.06$, $\mathrm{CHCl}_{3}$ ); IR (film) 2957, 2869, 1699, 11684, 1653, 1602, 1526, 1444, 1077, $743 \mathrm{~cm}^{-1}$; ${ }^{1} \mathrm{H}$ NMR (500 $\left.\mathrm{MHz}, \mathrm{C}_{6} \mathrm{D}_{6}\right) \delta 8.38$ (brs, 1H), 7.64-7.63 (m, 2H), 7.06-7.03 (m, 2H), $6.83(\mathrm{t}, 1 \mathrm{H}, J=7.3 \mathrm{~Hz}), 5.91-5.89$ (m, 1H), 5.23-5.21 (m, 1H), 5.11-5.09 (m, 1H), 4.56-4.55 (m, 1H), 1.34-1.02 (m, 4H), $0.74(\mathrm{t}, 1 \mathrm{H}, J=$ $7.3 \mathrm{~Hz}) ;{ }^{13} \mathrm{C}$ NMR $\left(125 \mathrm{MHz}, \mathrm{C}_{6} \mathrm{D}_{6}\right) \delta 168.5,130.7,129.2,126.7,124.1,119.1,87.7,86.2,38.7,19.0$, 14.0; HRMS (ESI): Exact mass calcd for $\mathrm{C}_{14} \mathrm{H}_{17} \mathrm{NO}_{2}[\mathrm{M}+\mathrm{H}]^{+}$, requires $m / z$ : 232.1337, found $m / z$ : 232.1330 .

(xiii) VanBrunt, M. P.; Standaert, R. F. Org. Lett. 2000, 2, 705. 'Syn' stereochemistry in the resultant dihydrofuran was determined by ${ }^{1 \mathrm{D}} \mathrm{NOE}$ and ${ }^{2 \mathrm{D}} \mathrm{NOESY}$ analyses. 


\section{Transformation of Vinyl Epoxide 10 to Dihydrofuran Derivative 13.} (2S,5R)-4-(tert-butyldiphenylsilyl)- $N$-phenyl-5-propyl-2,5-dihydrofuran-2-carboxamide (13): ${ }^{\text {iv }}$

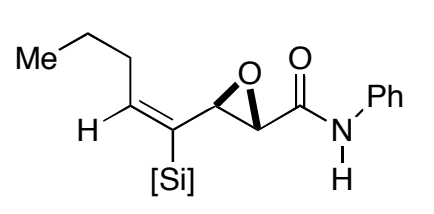

$[\mathrm{Si}]=$ TBDPS 10

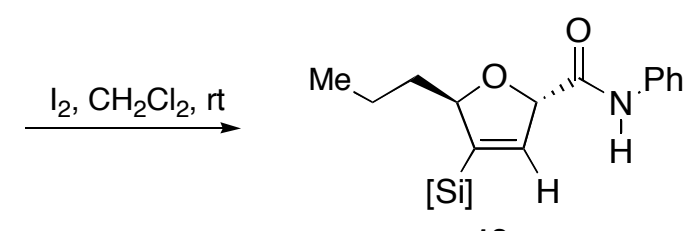

13

To a stirring mixture of vinyl epoxide $\mathbf{1 0}(100 \mathrm{mg}, 0.2 \mathrm{mmol})$ in $\mathrm{CH}_{2} \mathrm{Cl}_{2}(4.0 \mathrm{~mL})$ at $0{ }^{\circ} \mathrm{C}$ was added $\mathrm{I}_{2}$ (56 mg, $0.22 \mathrm{mmol}$ ). After $5 \mathrm{~min}$, the ice bath was removed and the reddish brown solution was stirred at room temperature overnight. The reaction mixture was diluted with $\mathrm{CH}_{2} \mathrm{Cl}_{2}$ and stirred vigorously. To this, a saturated aqueous solution of $\mathrm{Na}_{2} \mathrm{~S}_{2} \mathrm{O}_{3}$ was added dropwise until the solution became colourless. The mixture was phase-separated and the aqueous layer was back extracted with $\mathrm{CH}_{2} \mathrm{Cl}_{2}$ (x2). The combined organic layers were dried using $\mathrm{MgSO}_{4}$. After evaporation under reduced pressure, the crude product was subjected to ${ }^{1} \mathrm{H}$ NMR analysis and was detected as a single diastereomer. The crude product was purified by silica gel flash column chromatography (7\% EtOAc/Hexanes) to give dihydropyran derivative $\mathbf{1 3}$ as a white foamy solid that was homogeneous as judged by ${ }^{1} \mathrm{H}$ NMR spectroscopy (98\% yield): $\mathrm{mp} 123-124{ }^{\circ} \mathrm{C} ;[\alpha]_{\mathrm{D}}-280\left(c=0.71, \mathrm{CHCl}_{3}\right.$ ); IR (film) 3377, 3069, 2959, 2859, 1687, 1600, 1525, 1499, 1442, 1316, 1106, 817, 740, $701 \mathrm{~cm}^{-1} ;{ }^{1} \mathrm{H}$ NMR (500 MHz, $\mathrm{CDCl}_{3}$ ) $\delta 8.44$ (brs, 1H), 7.62$7.57(\mathrm{~m}, 6 \mathrm{H}), 7.44-7.33(\mathrm{~m}, 8 \mathrm{H}), 7.15(\mathrm{t}, 1 \mathrm{H}, J=7.3 \mathrm{~Hz}), 6.57(\mathrm{t}, 1 \mathrm{H}, J=1.8 \mathrm{~Hz}), 5.36(\mathrm{dd}, 1 \mathrm{H}, J=1.5$ $\mathrm{Hz}, 2.7 \mathrm{~Hz}), 5.16-5.13(\mathrm{~m}, 1 \mathrm{H}), 1.32-1.25(\mathrm{~m}, 2 \mathrm{H}), 1.21-1.14(\mathrm{~m}, 2 \mathrm{H}), 11.11(\mathrm{~s}, 9 \mathrm{H}), 0.61(\mathrm{t}, 3 \mathrm{H}, J=6.8$ $\mathrm{Hz}) ;{ }^{13} \mathrm{C}$ NMR (125 MHz, $\left.\mathrm{CDCl}_{3}\right) \delta$ 217.4, 169.2, 139.2, 137.5, 136.5, 133.4, 130., 129.8, 128.0, 124.7, 122.2, 120.1, 93.7, 86.9, 37.7, 30.5, 28.4, 18.9, 18.8, 13.7; HRMS (ESI): Exact mass calcd for $\mathrm{C}_{30} \mathrm{H}_{36} \mathrm{NO}_{2} \mathrm{Si}[\mathrm{M}+\mathrm{H}]^{+}$, requires $m / z: 470.2515$, found $m / z: 490.2506$; Enantiomeric excess determined by HPLC with Chiracel ADH column, $1 \% i$-PrOH/hexanes, $0.5 \mathrm{~mL} / \mathrm{min}, 254 \mathrm{~nm} ; \mathrm{t}_{\mathrm{r}}$ (major) $=19.2, \mathrm{t}_{\mathrm{r}}$ $($ minor $)=31.5,94 \%$ ee .

(xiv) 'Anti' stereochemistry in $\mathbf{1 3}$ was determined by ${ }^{1 \mathrm{D}} \mathrm{NOE}$ and ${ }^{2 \mathrm{D}} \mathrm{NOESY}$ analyses. 
Mosher's ester analysis of $[2 S,(3,4,5 S)]-2$-hydroxy- $N$-phenylocta-3,4-dienamide 12:

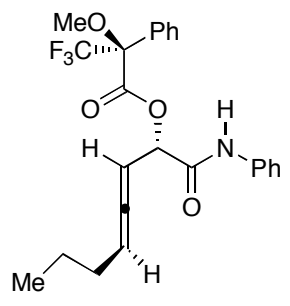

${ }^{1} \mathrm{H}$ NMR (500 MHz, $\left.\mathrm{CDCl}_{3}\right) \delta 7.596(\mathrm{~d}, 2 \mathrm{H}, J=7.33 \mathrm{~Hz}), 7.485-7.4011-7.428(\mathrm{~m}$, 5H), 7.393 (brs, 1H), 7.386-7.284 (m, 2H), 7.137-7.103 (m, 1H), 5.931 (dd, 1H, J= $2.69 \mathrm{~Hz}, 3.91 \mathrm{~Hz}), 5.479-5.450$ (m, 2H), 3.627 (s, 3H), 2.032-1.983 (m, 2H), 1.457$1.379(\mathrm{~m}, 2 \mathrm{H}), 0.894(\mathrm{t}, 3 \mathrm{H}, J=7.32 \mathrm{~Hz})$.

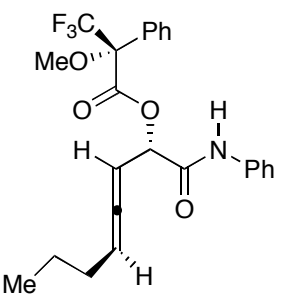

${ }^{1} \mathrm{H}$ NMR (500 MHz, $\mathrm{CDCl}_{3}$ ) $\delta 7.663$ (brs, 1H), 7.584 (d, 2H, J = $7.81 \mathrm{~Hz}$ ), 7.468$7.428(\mathrm{~m}, 5 \mathrm{H}), 7.332(\mathrm{t}, 2 \mathrm{H}, J=7.81 \mathrm{~Hz}), 7.147(\mathrm{t}, 1 \mathrm{H}, J=7.32 \mathrm{~Hz}), 5.848(\mathrm{dd}, 1 \mathrm{H}$, $J=2.44 \mathrm{~Hz}, 3.42 \mathrm{~Hz}), 5.470-5.396(\mathrm{~m}, 2 \mathrm{H}), 3.587$ (s, 3H), 2.354-1.958 (m, 2H), 1.440-1.356 (m, 2H), $0.885(\mathrm{t}, 3 \mathrm{H}, J=7.32 \mathrm{~Hz})$. 
1H NMR SPECTRA (in $\mathrm{CDCl}_{3}$ unless otherwise indicated)

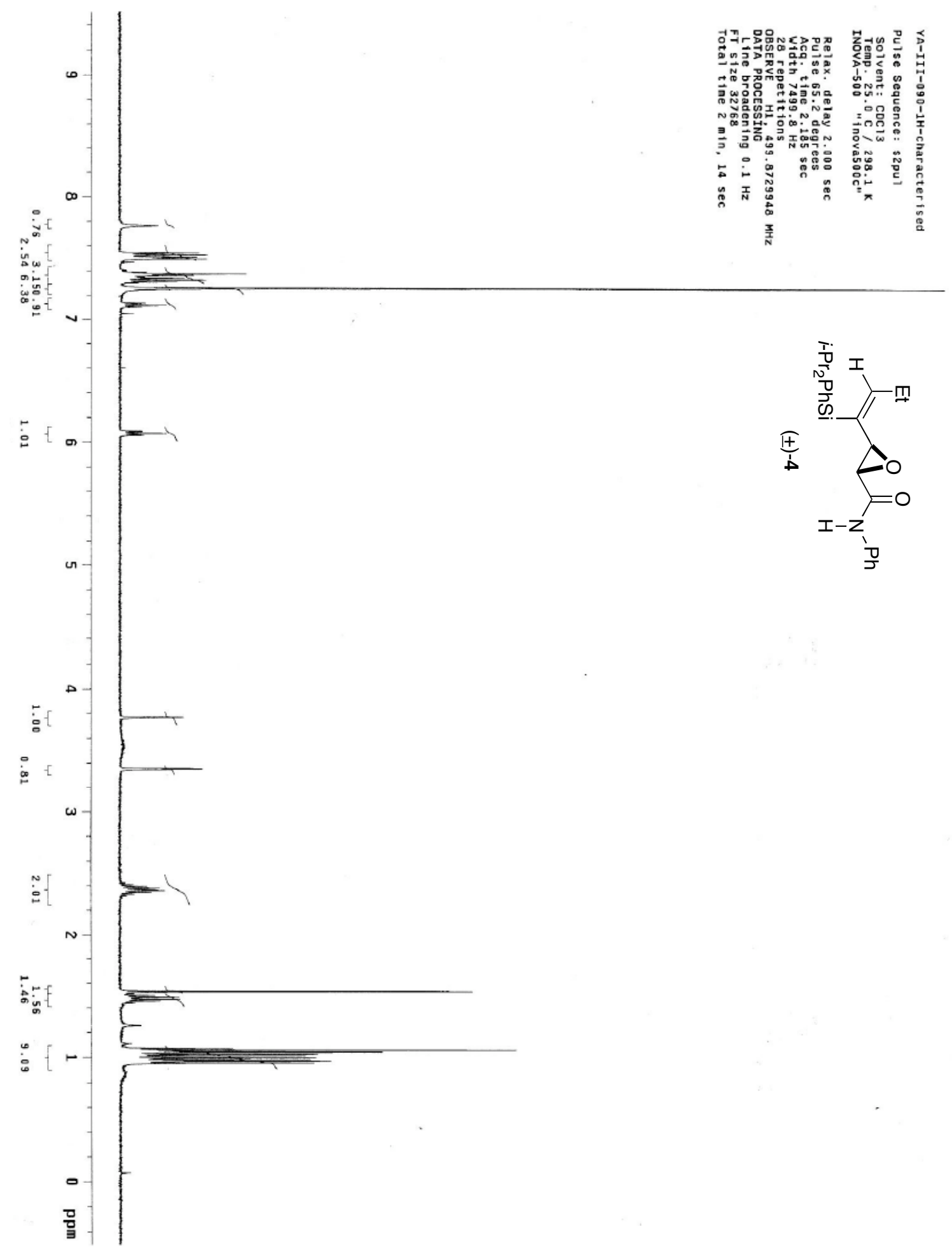




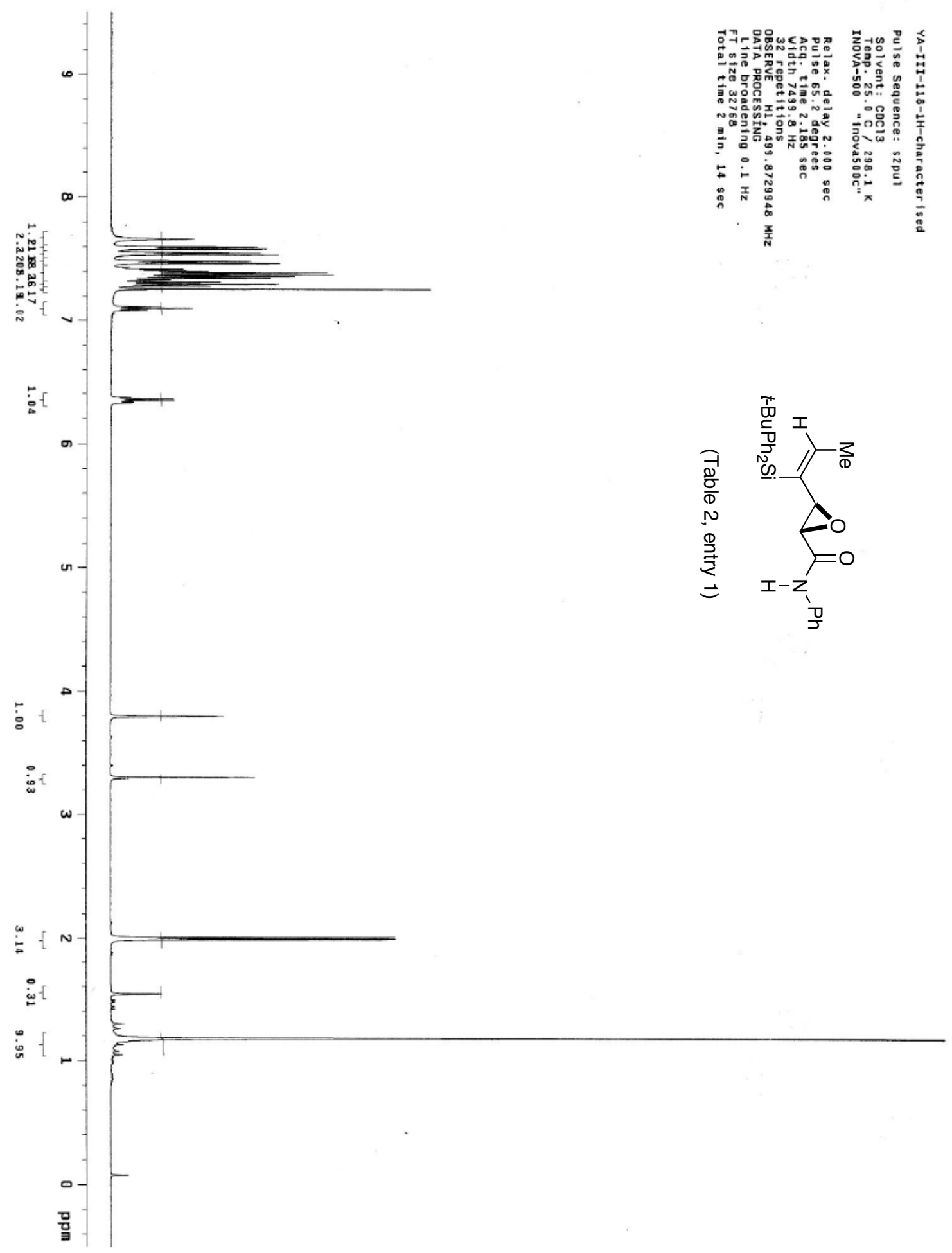




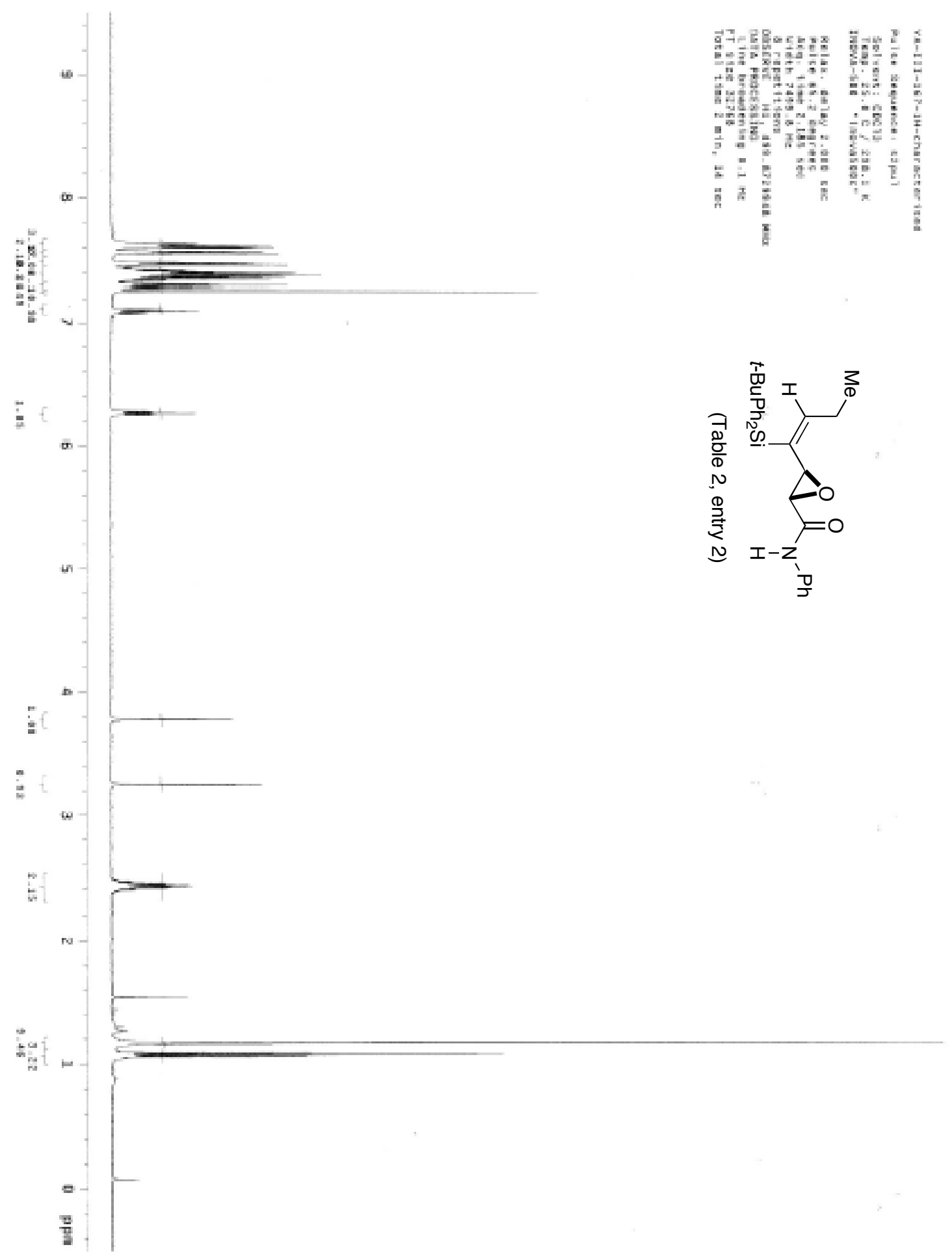




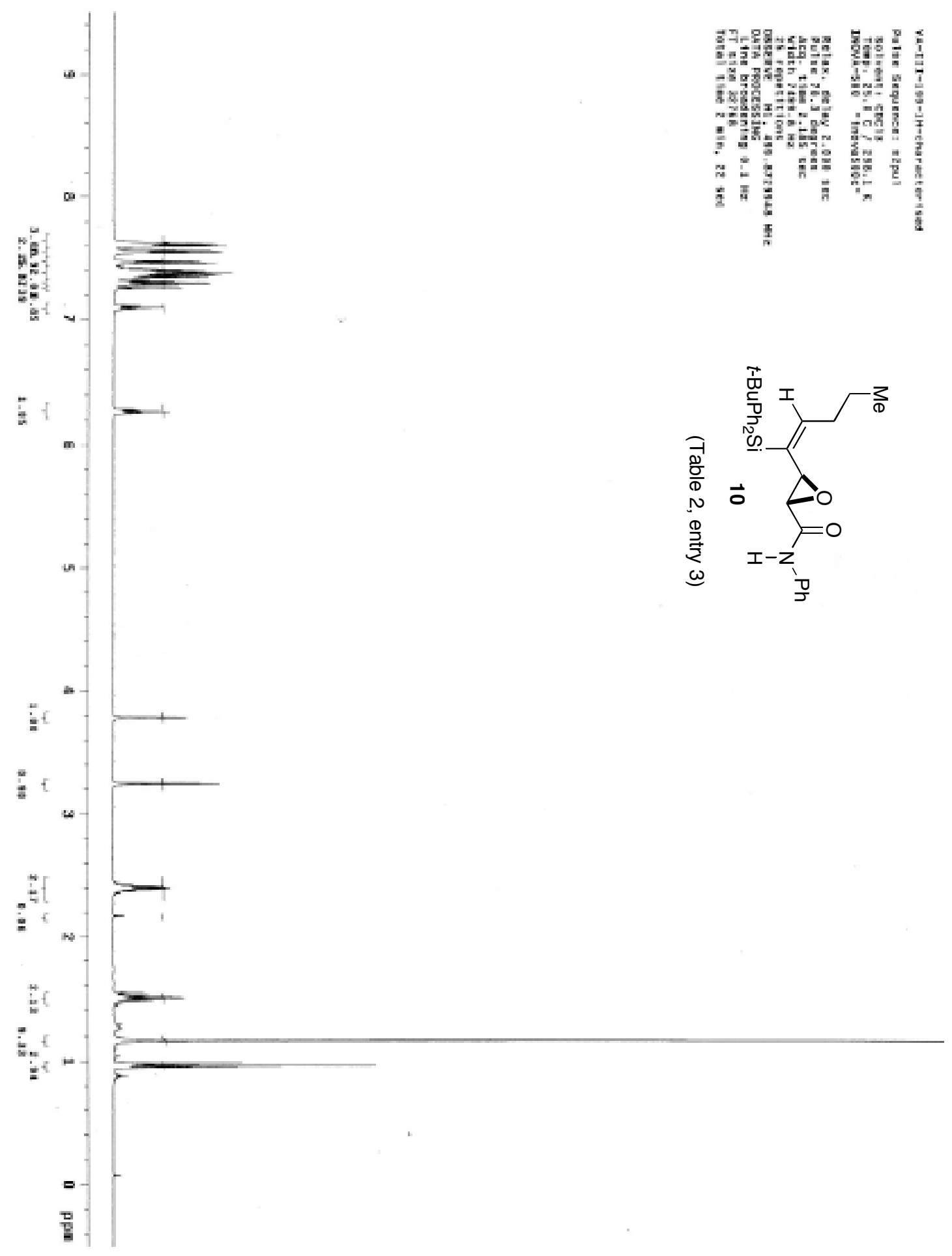




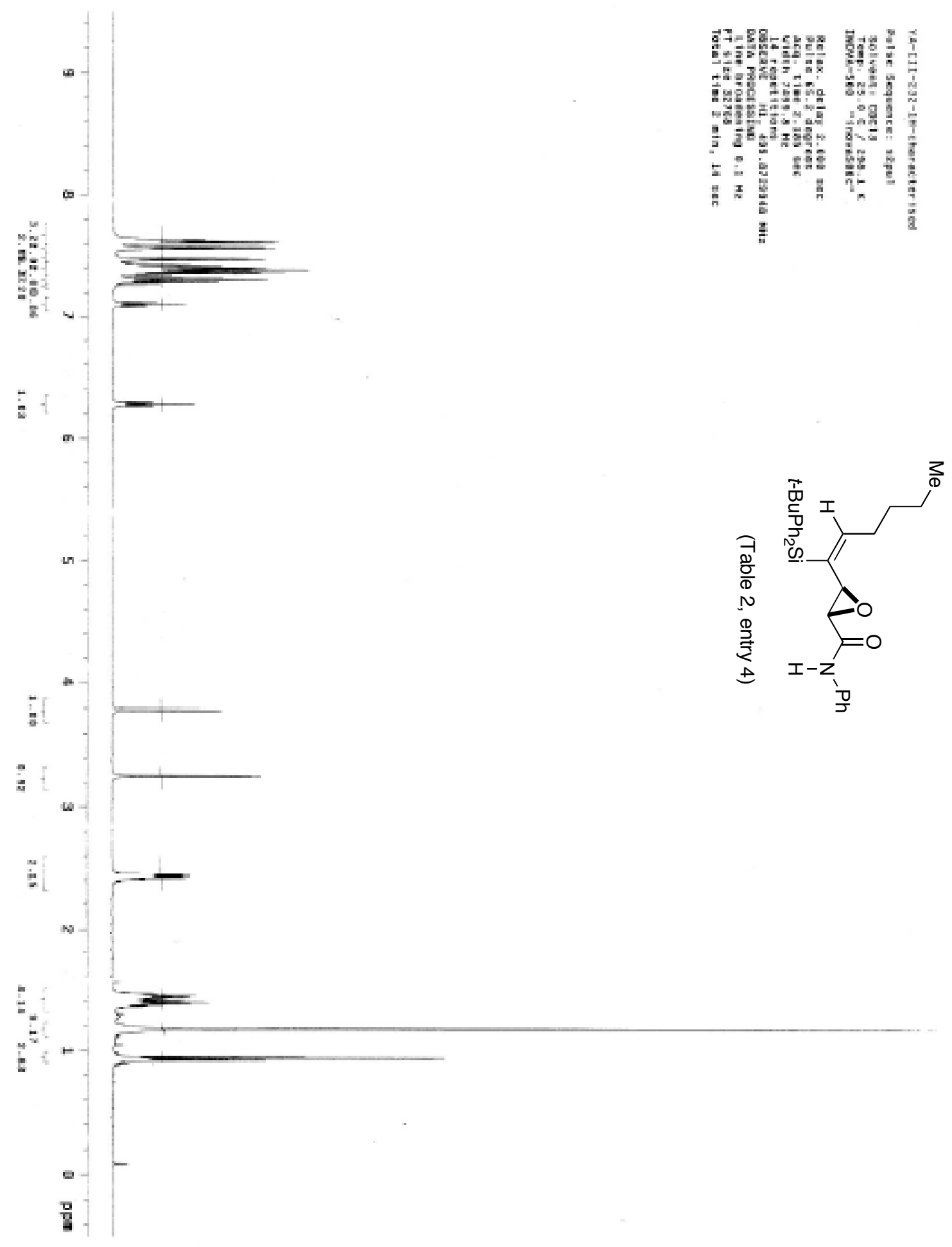




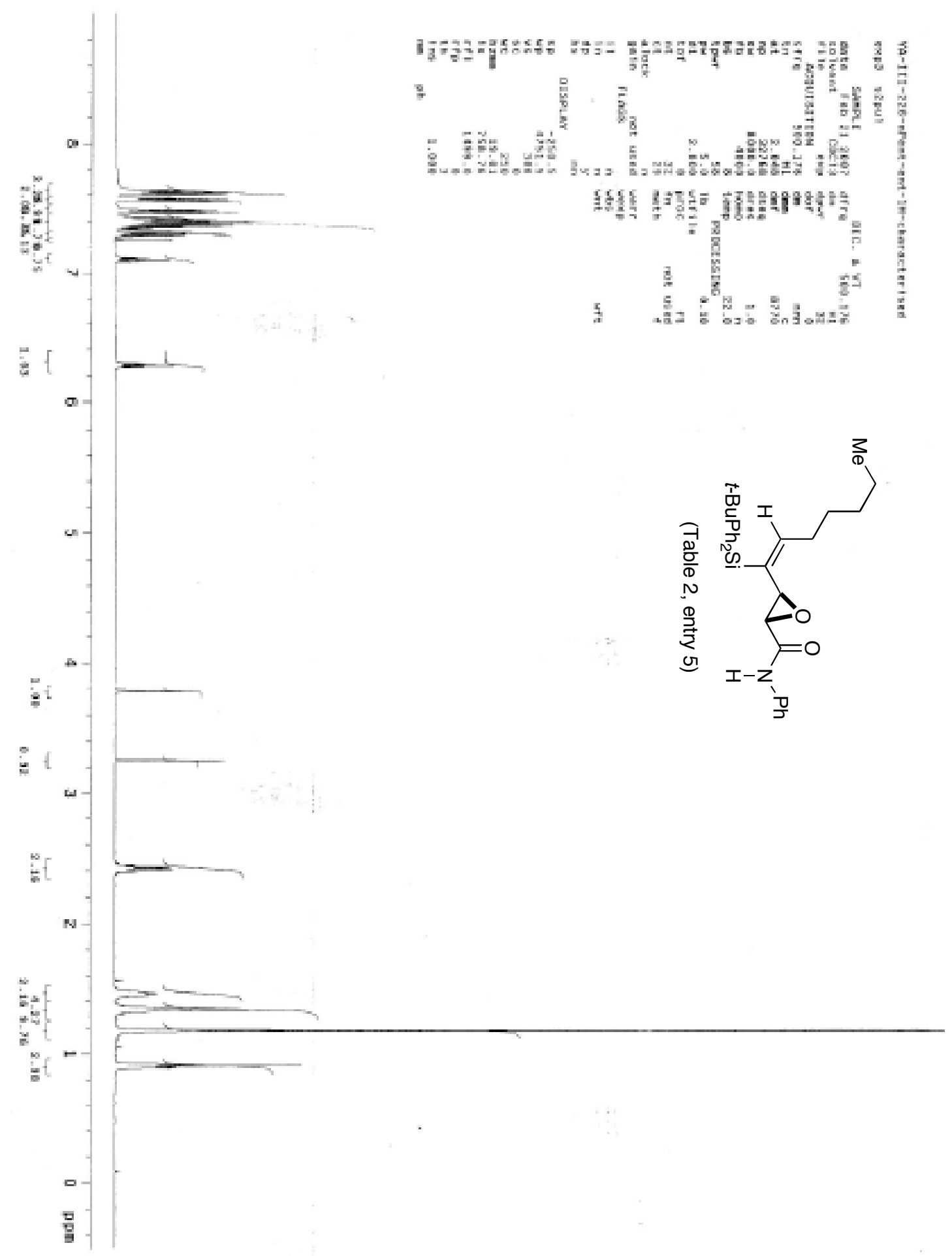




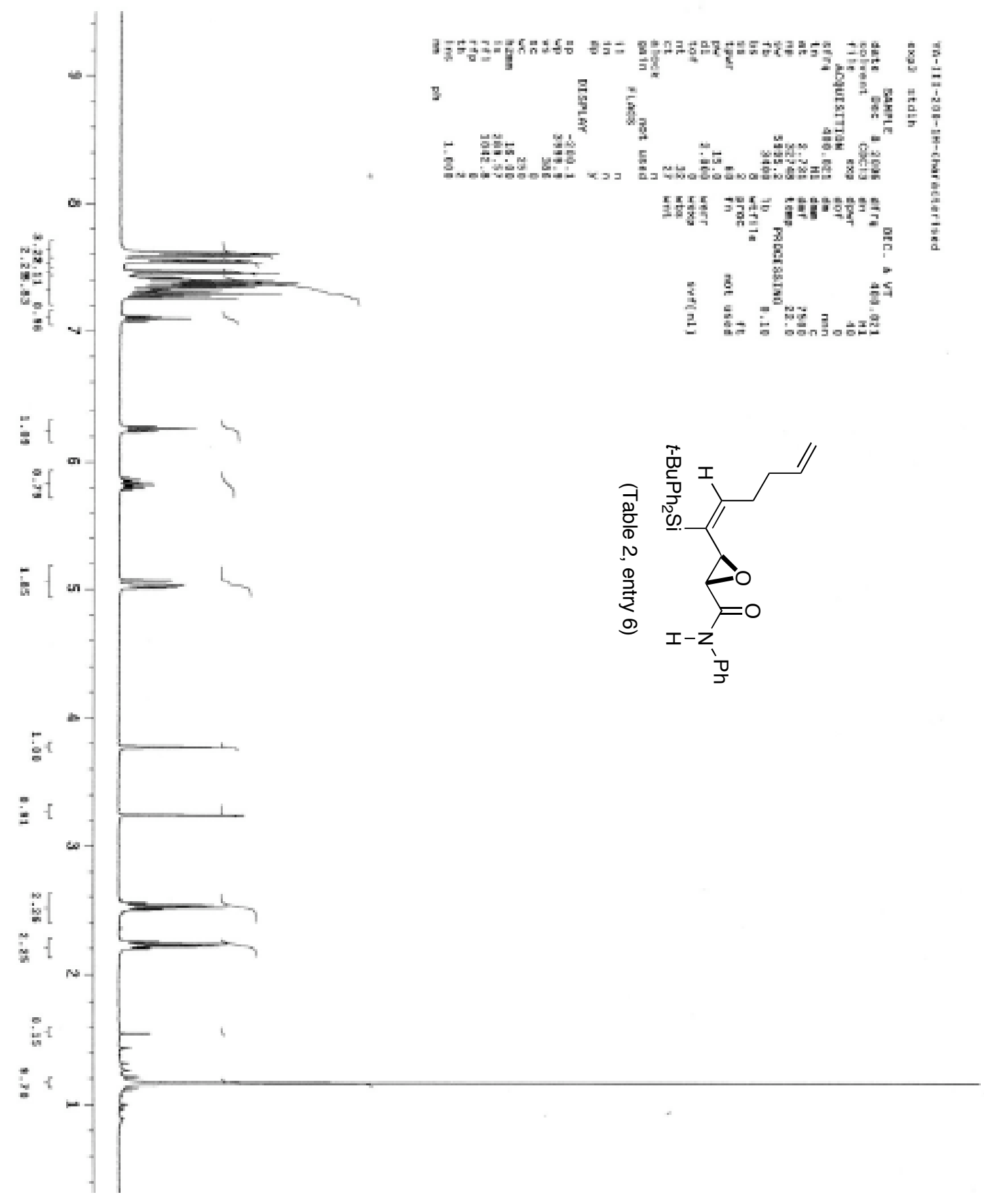




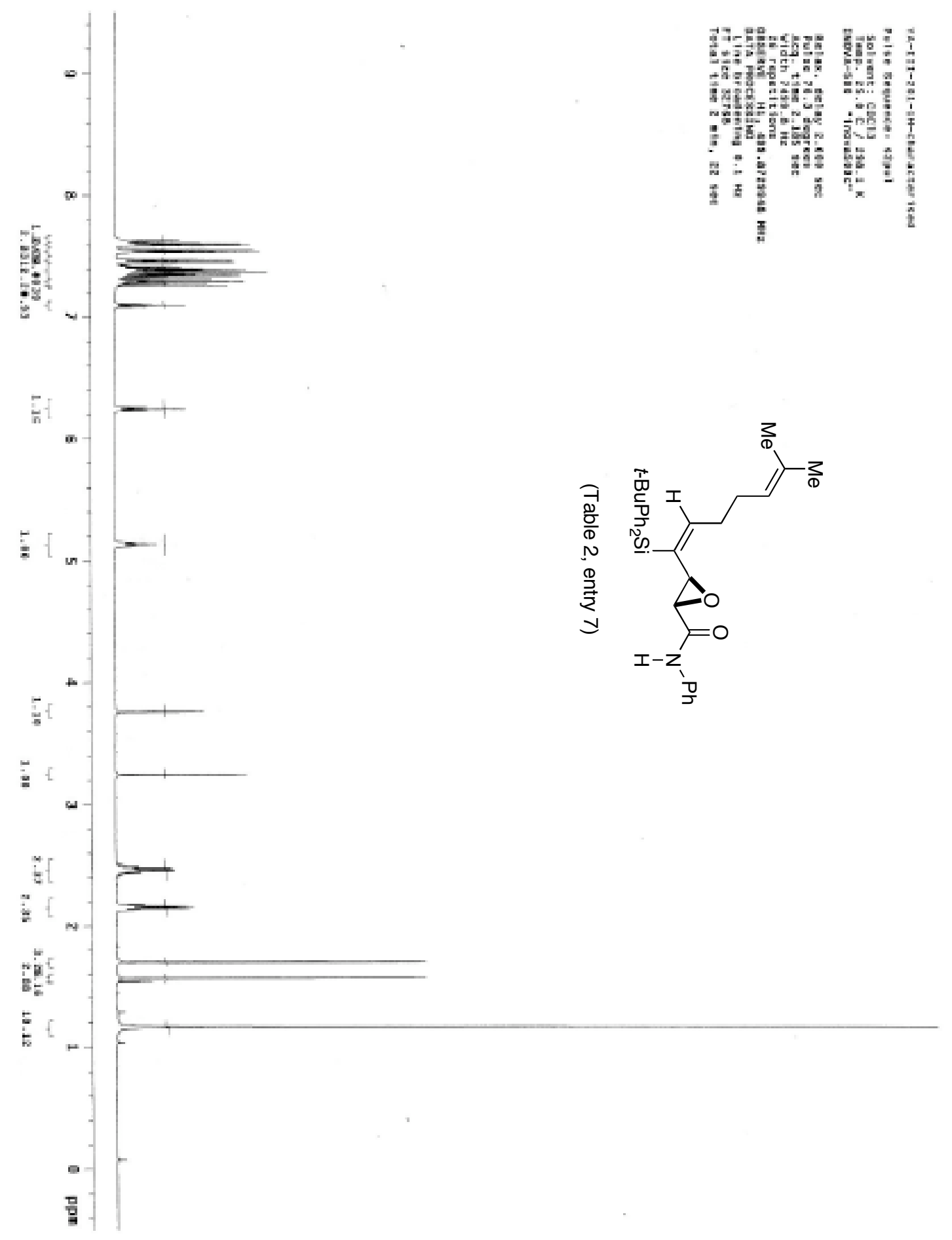




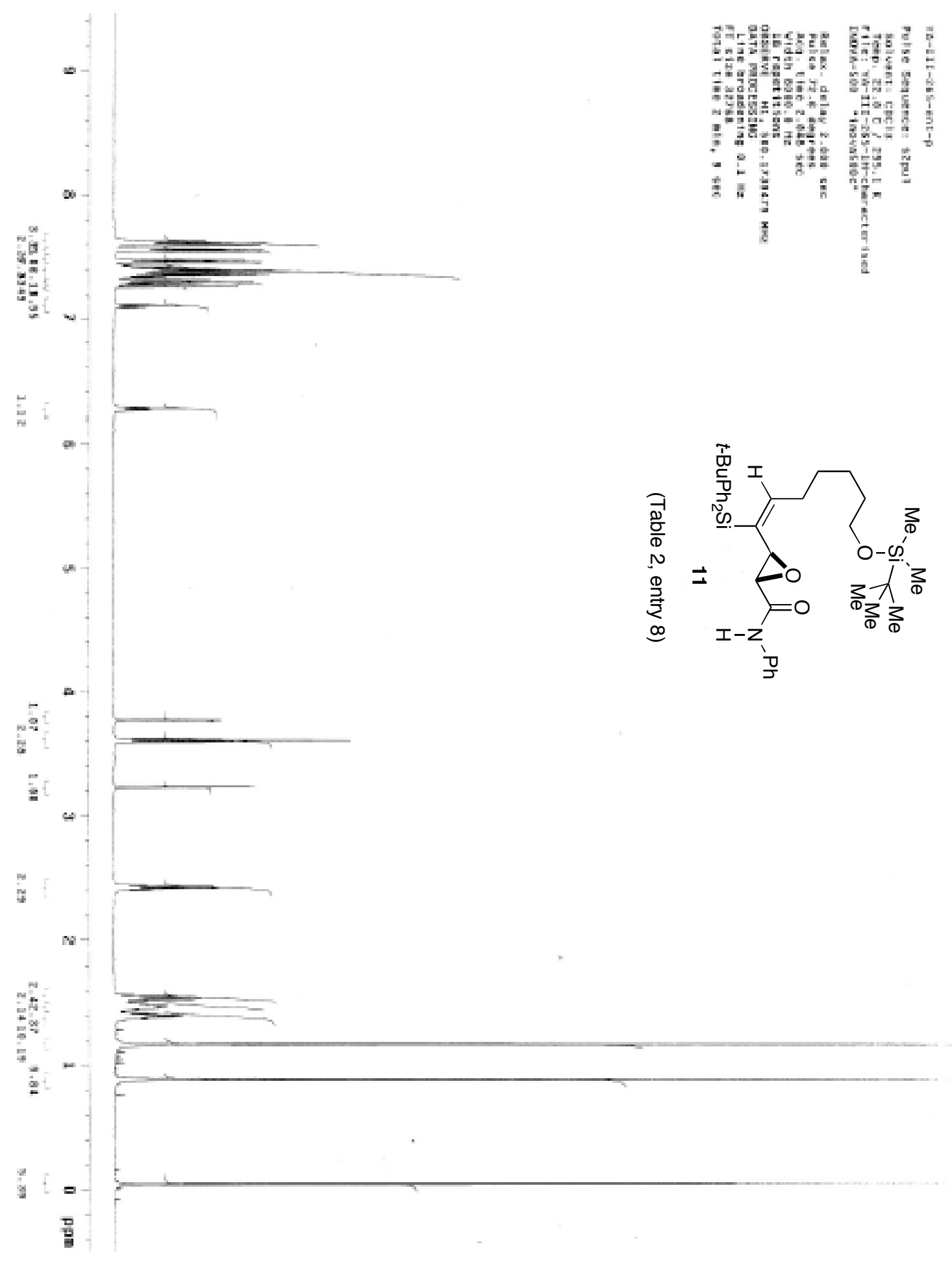




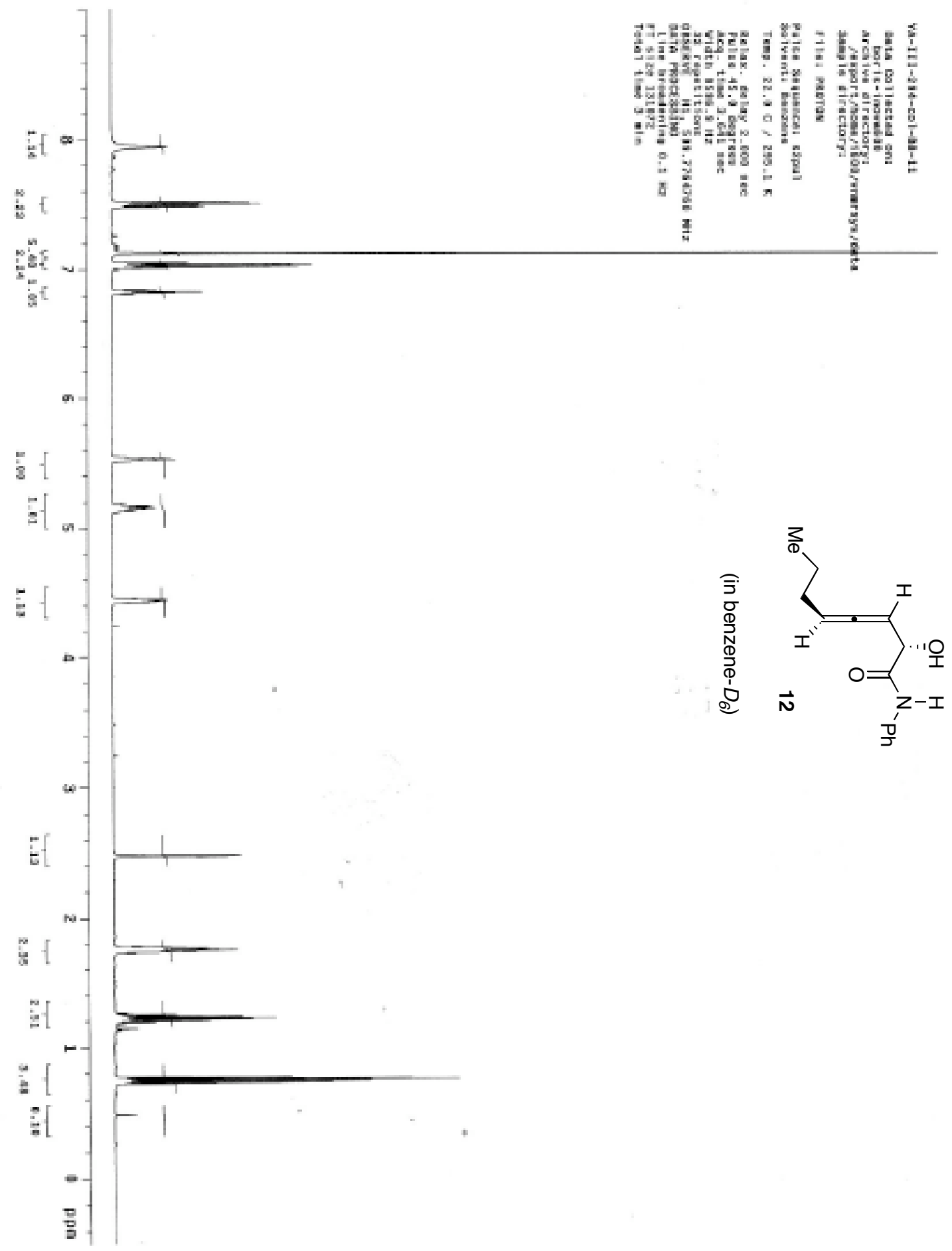




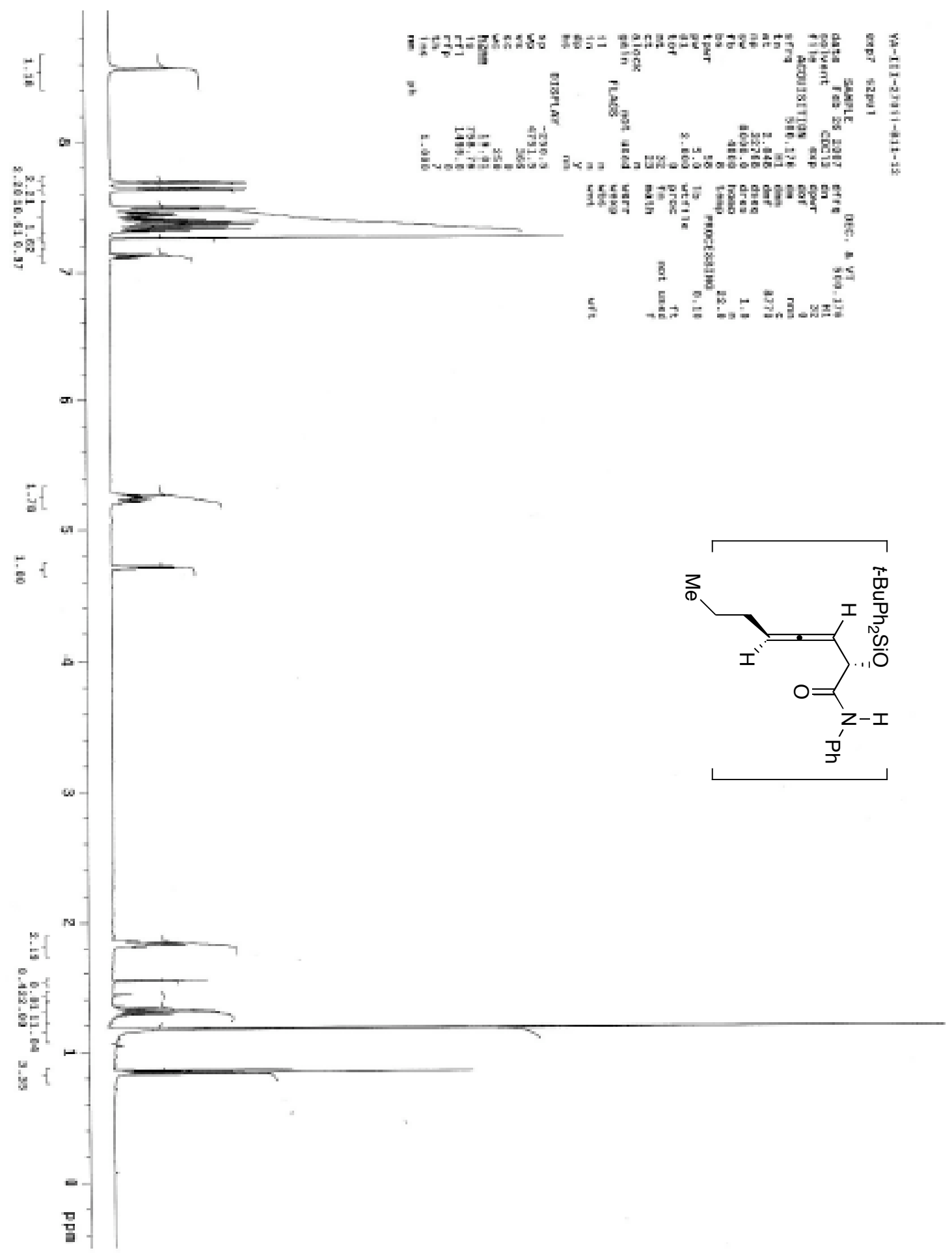




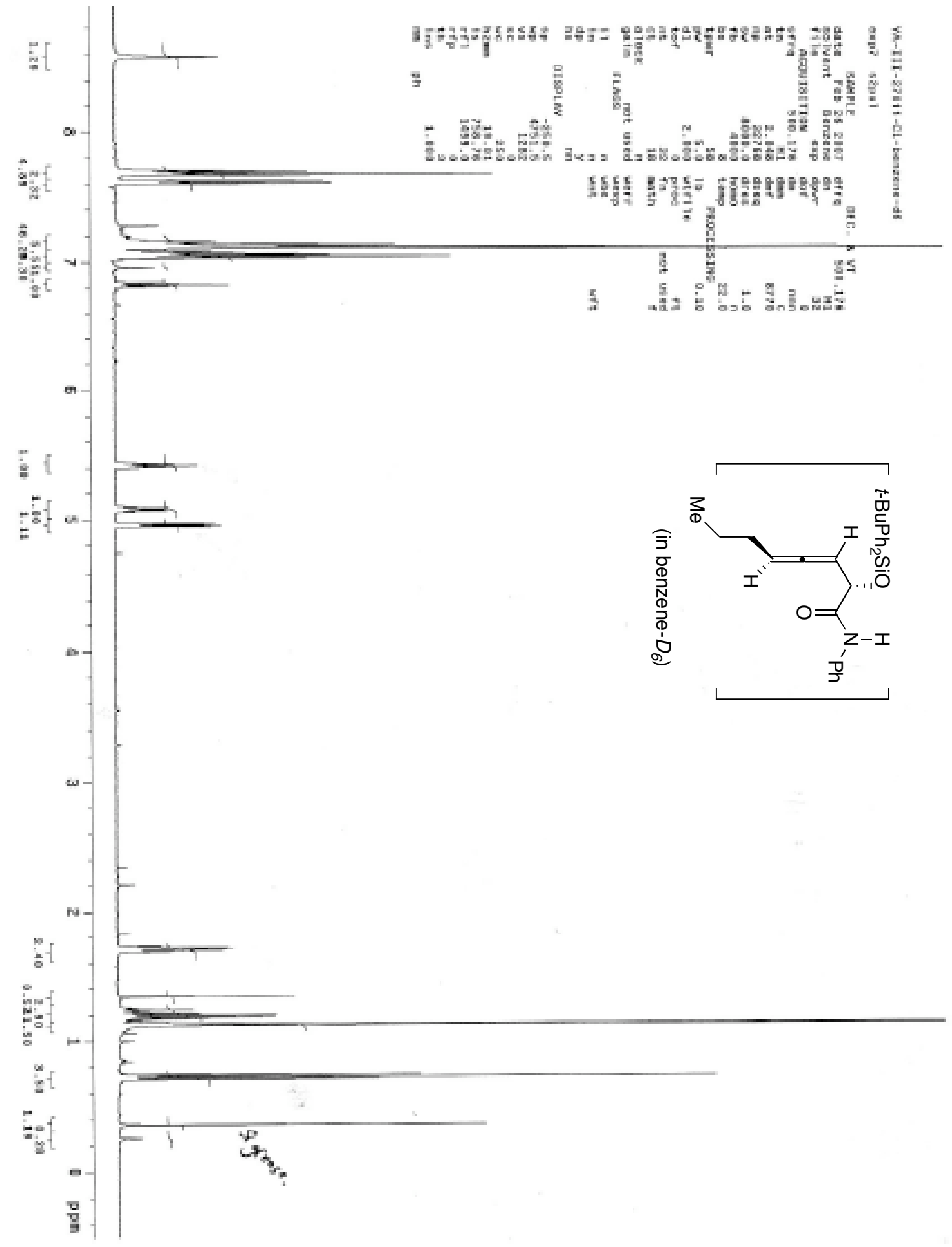




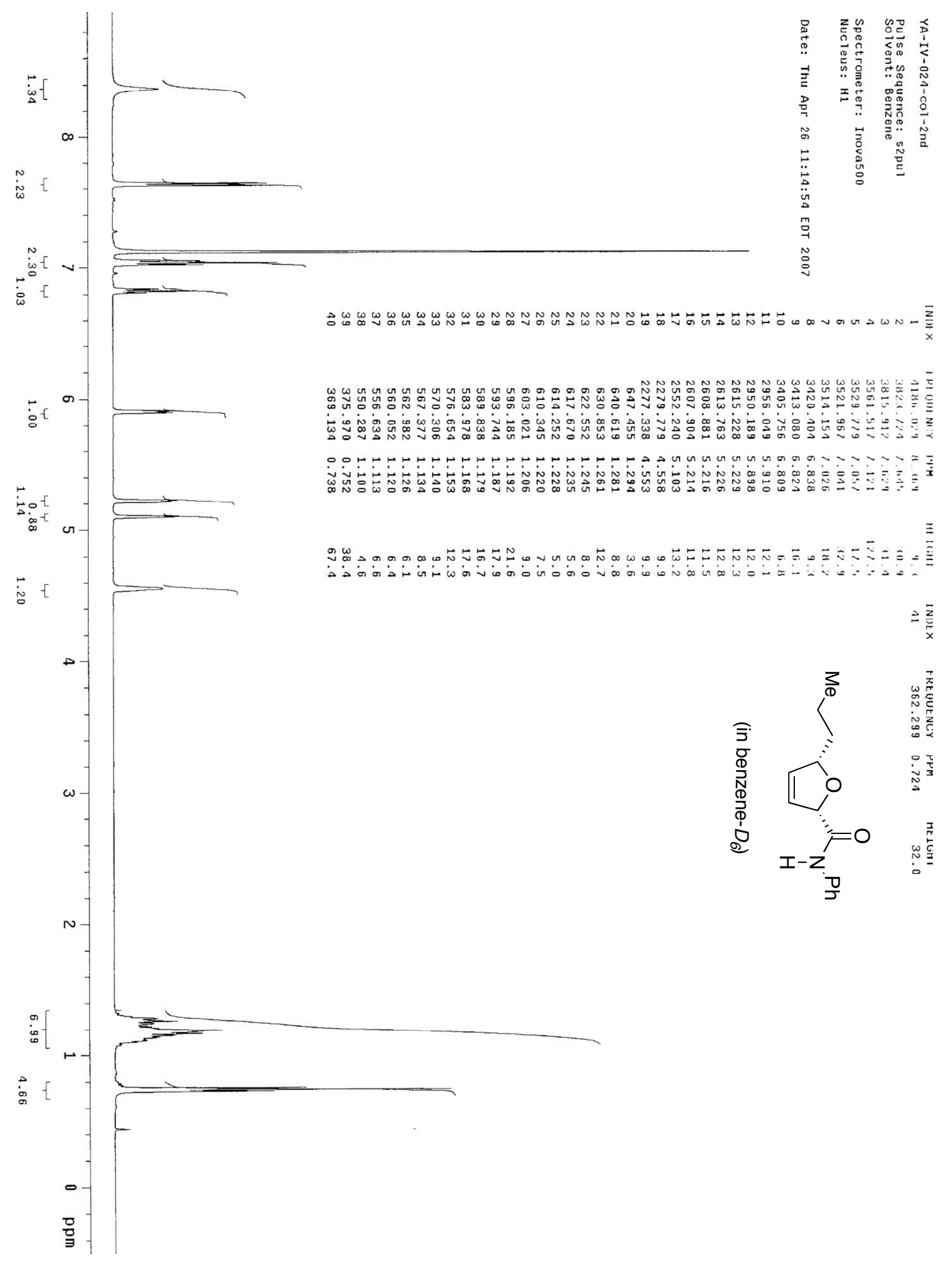




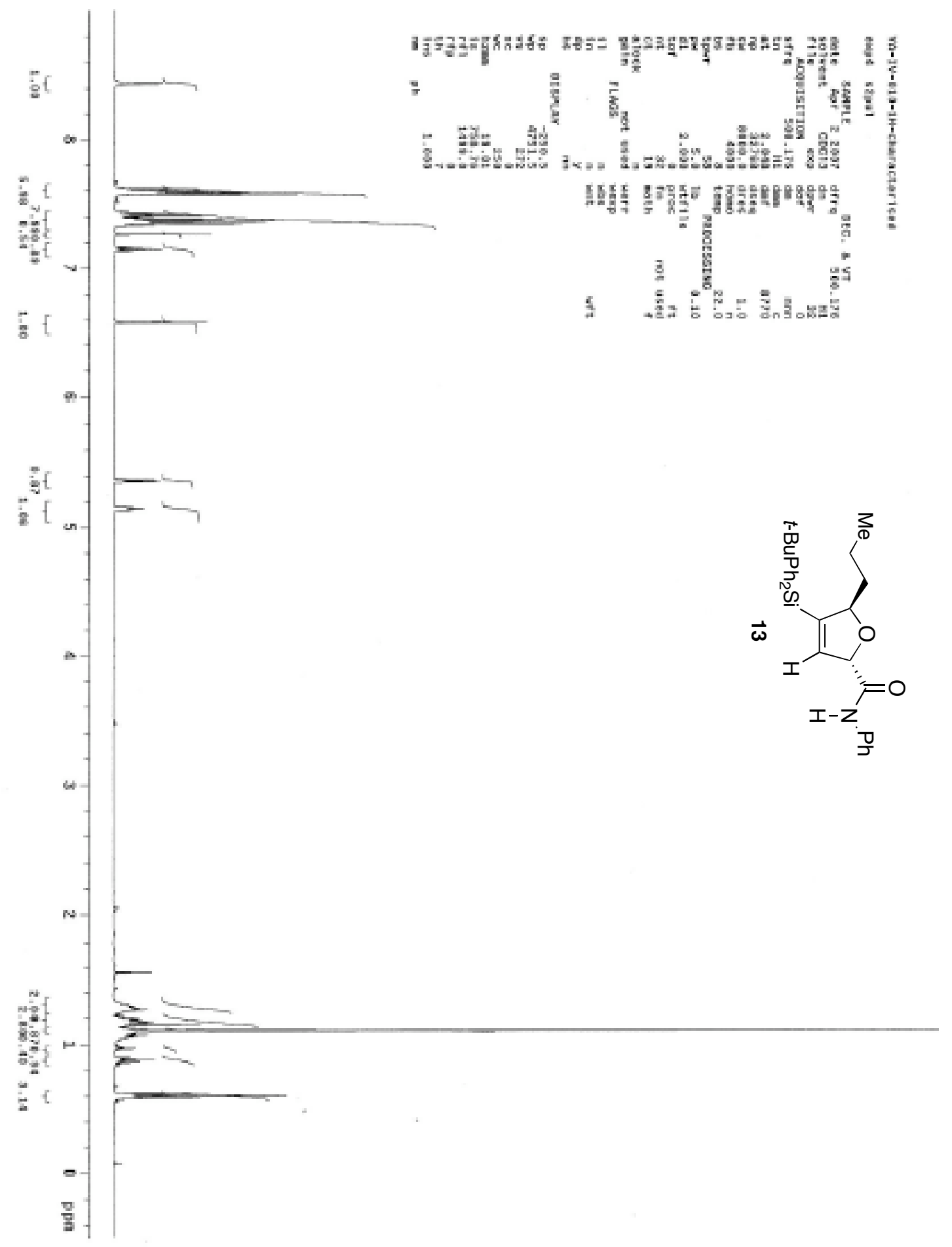


13C NMR SPECTRA (in $\mathrm{CDCl}_{3}$ unless otherwise indicated)

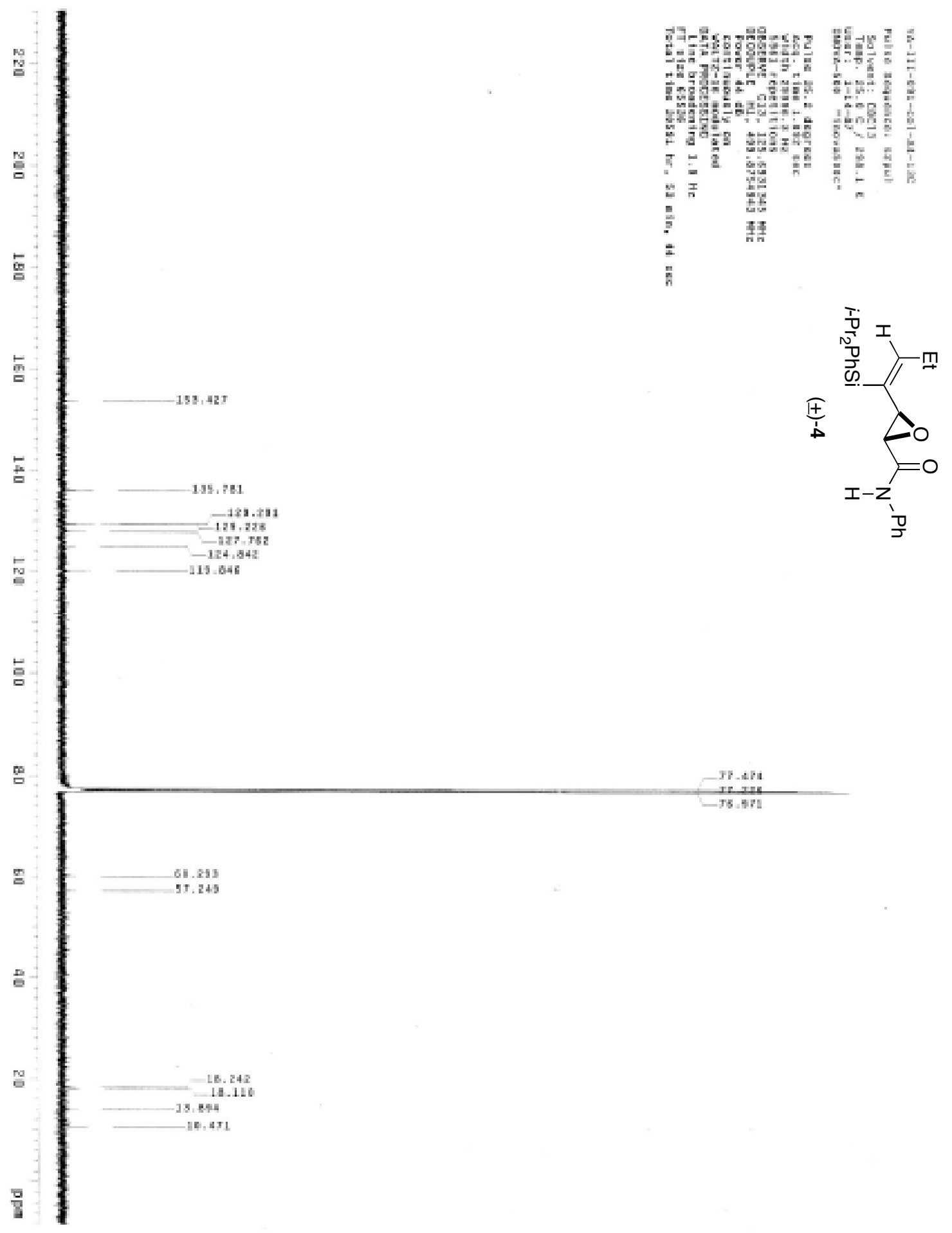




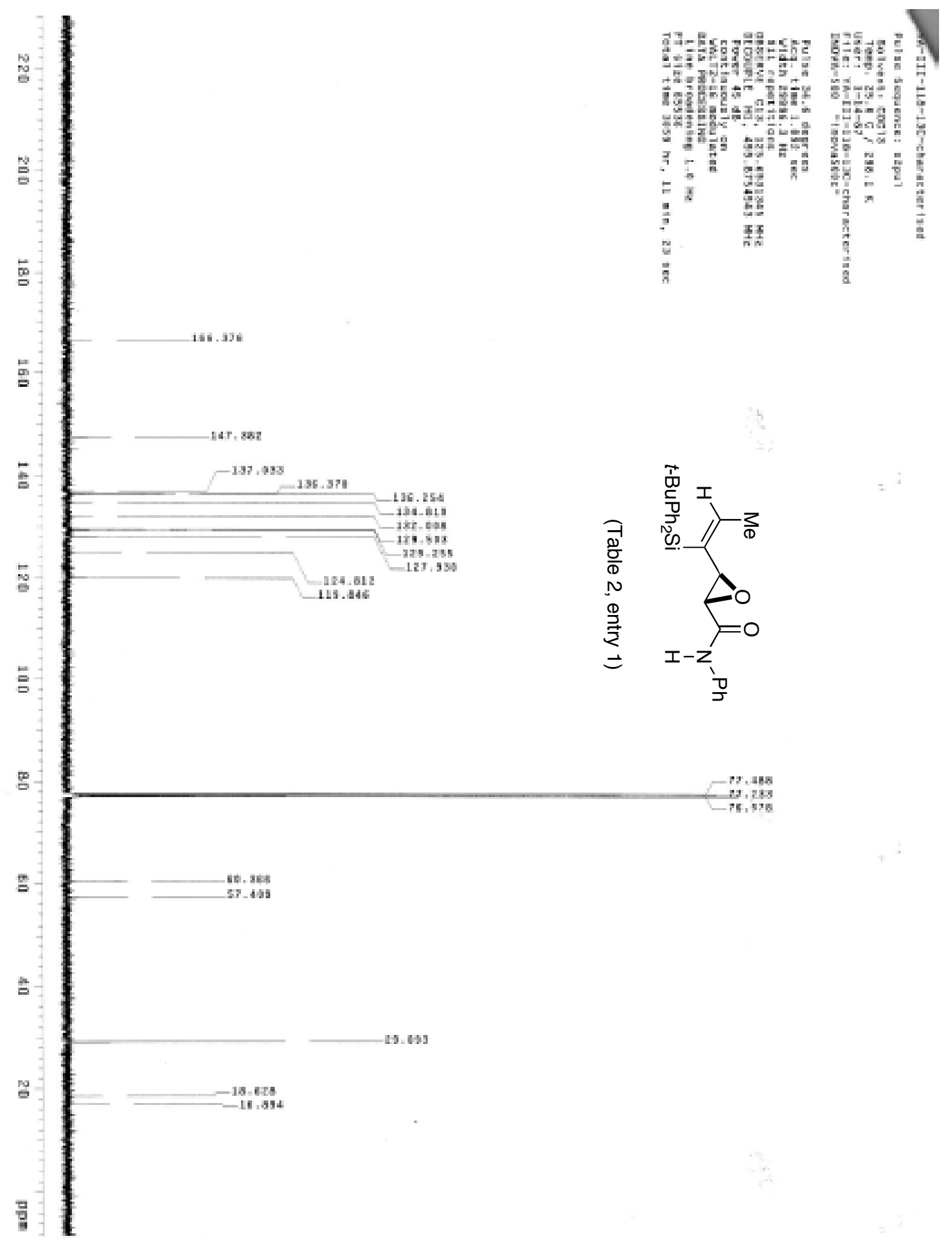




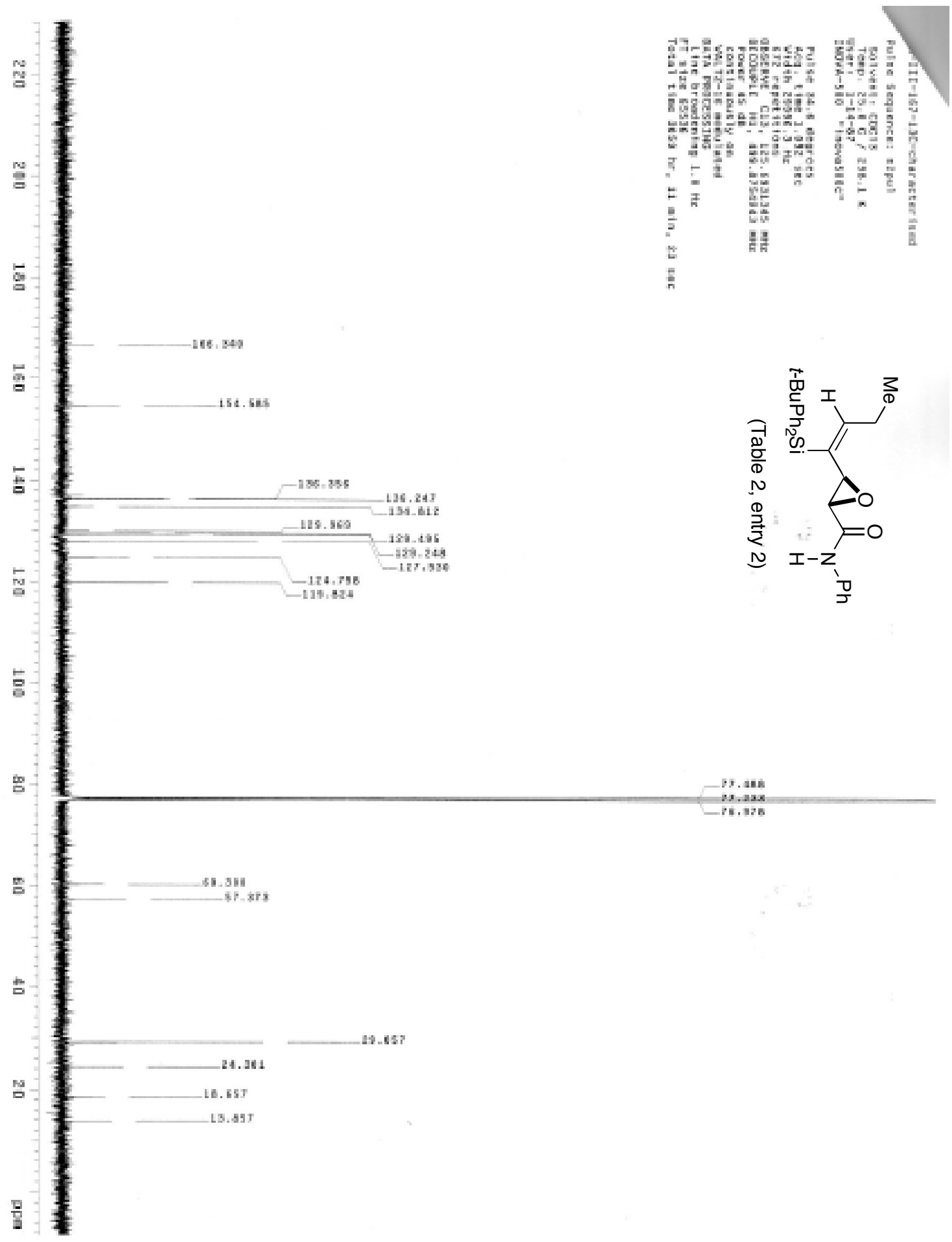




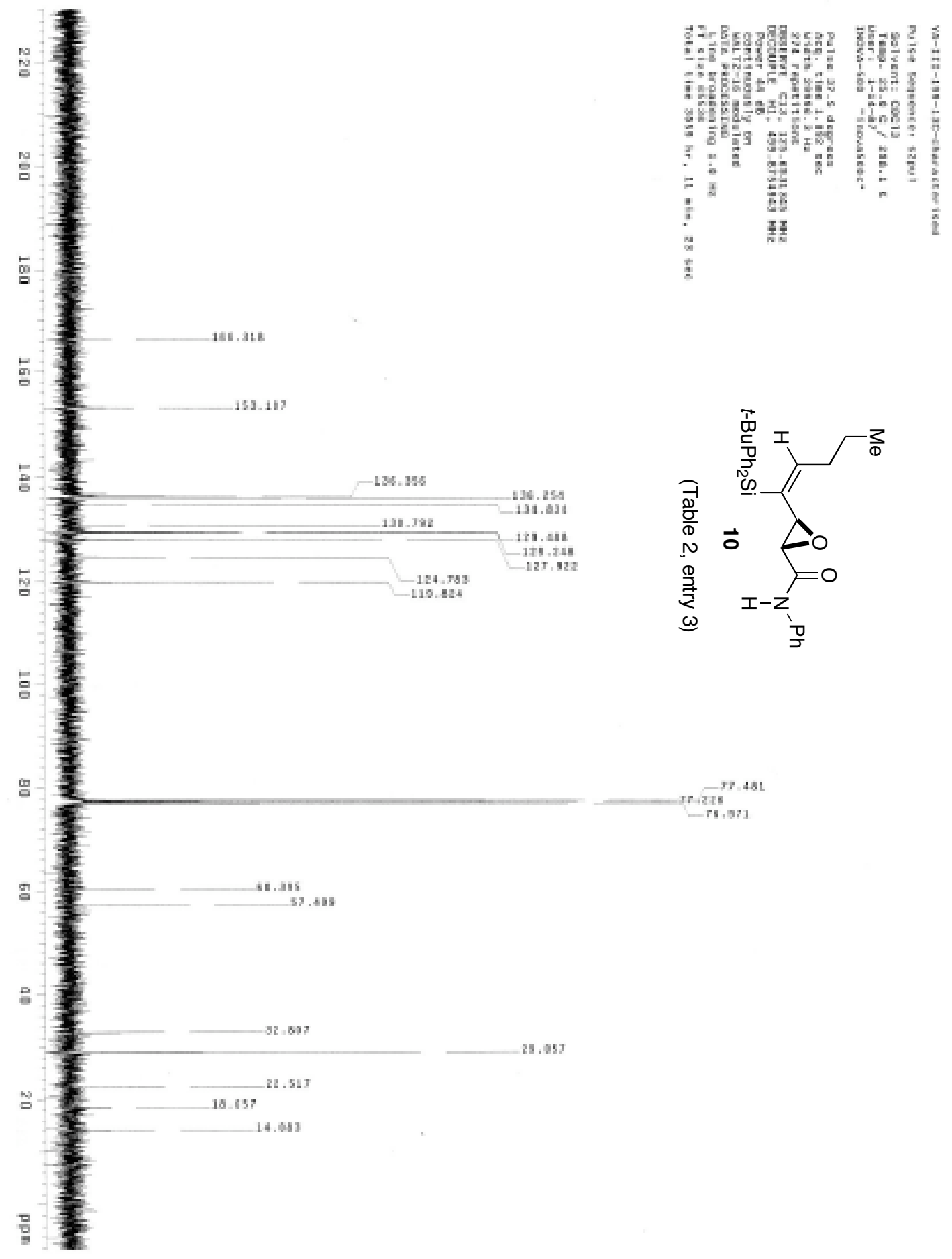




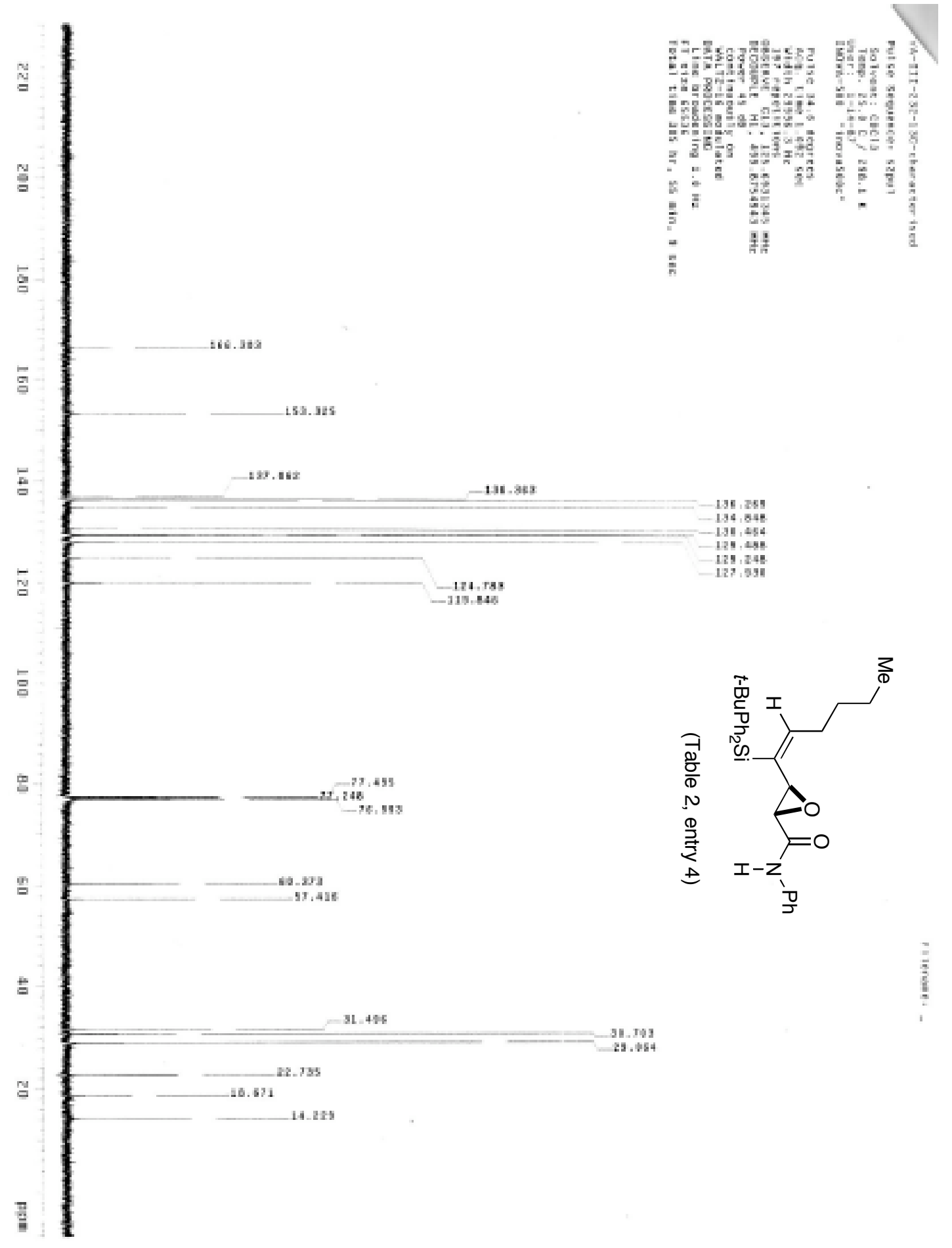




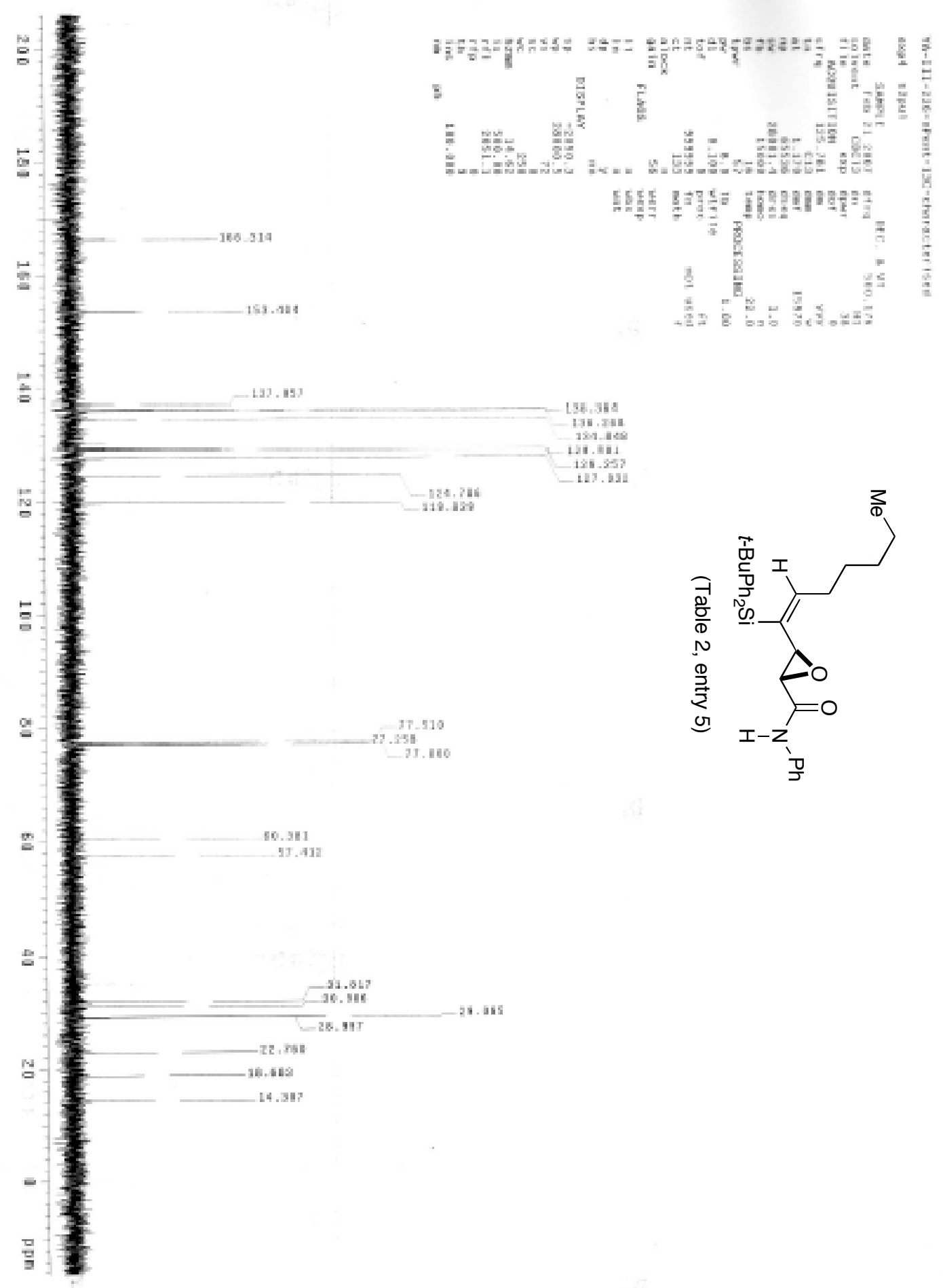




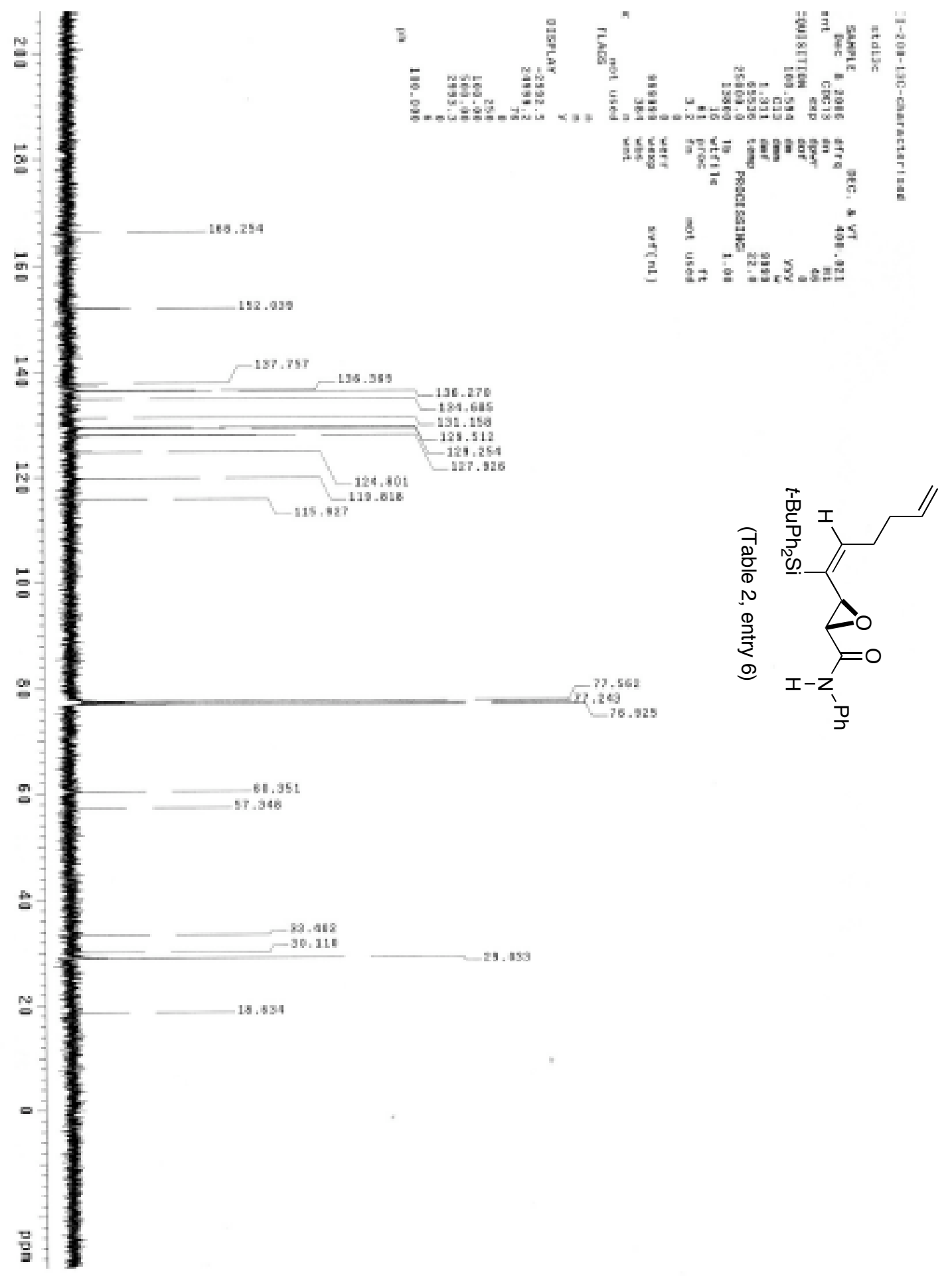




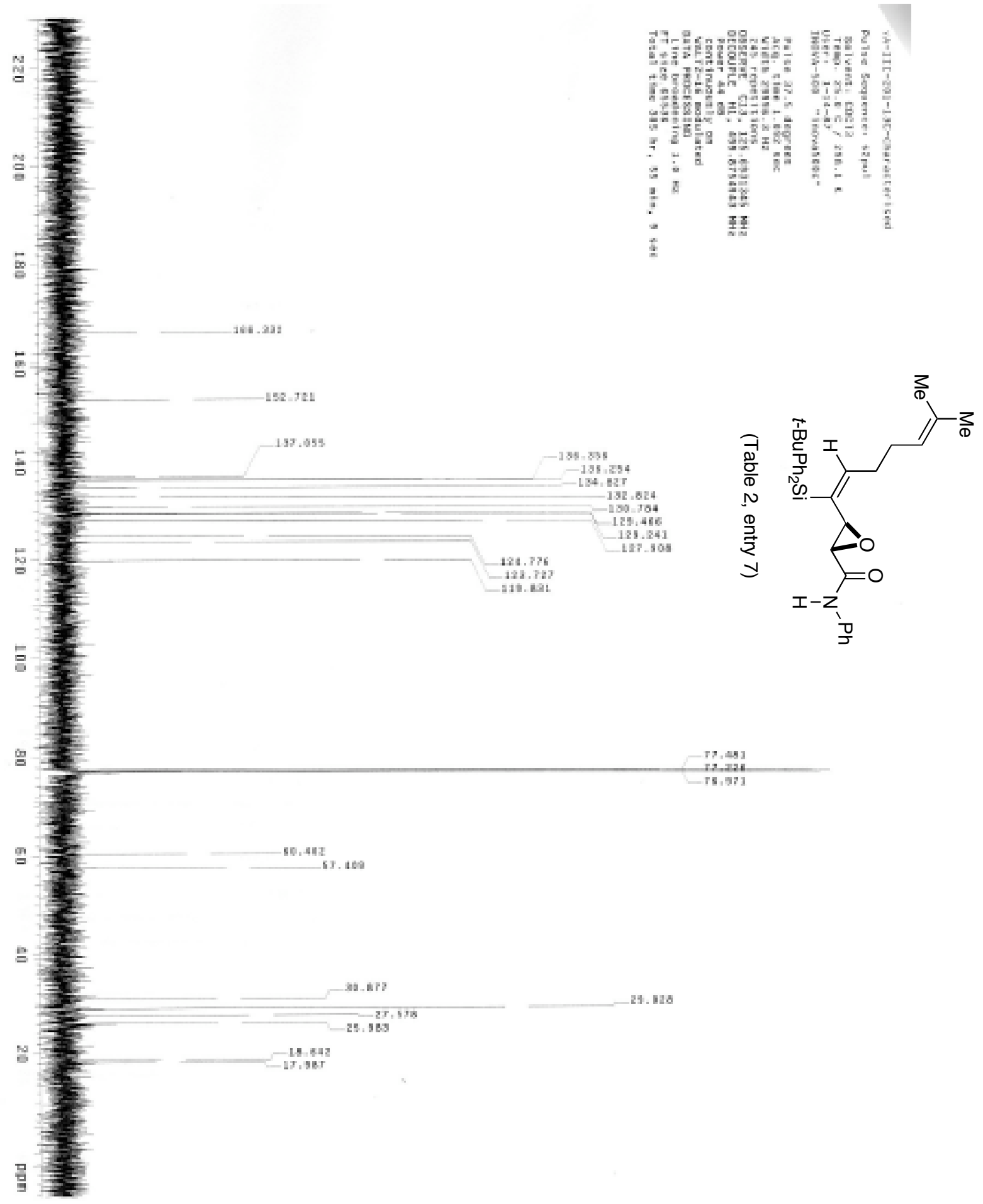




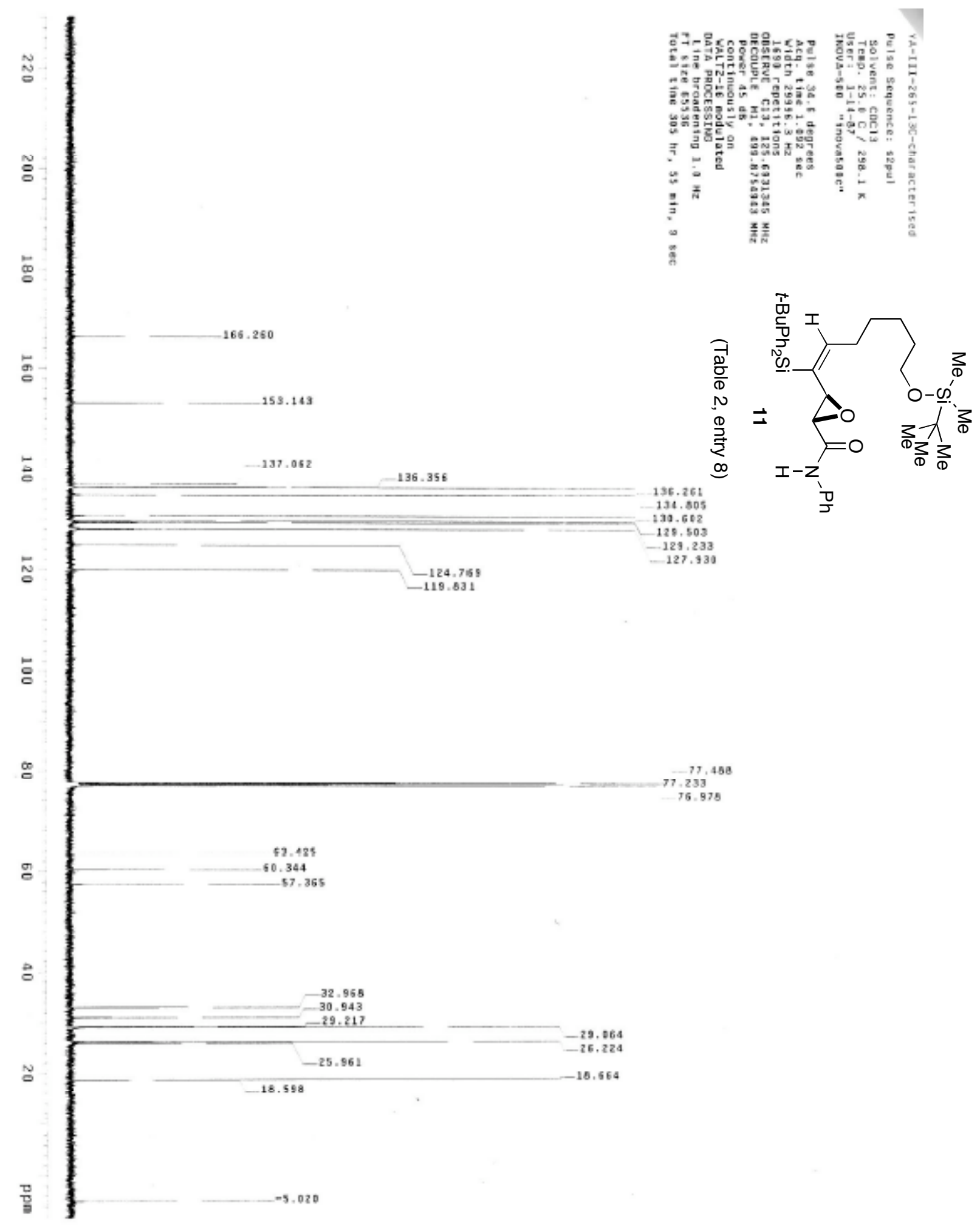




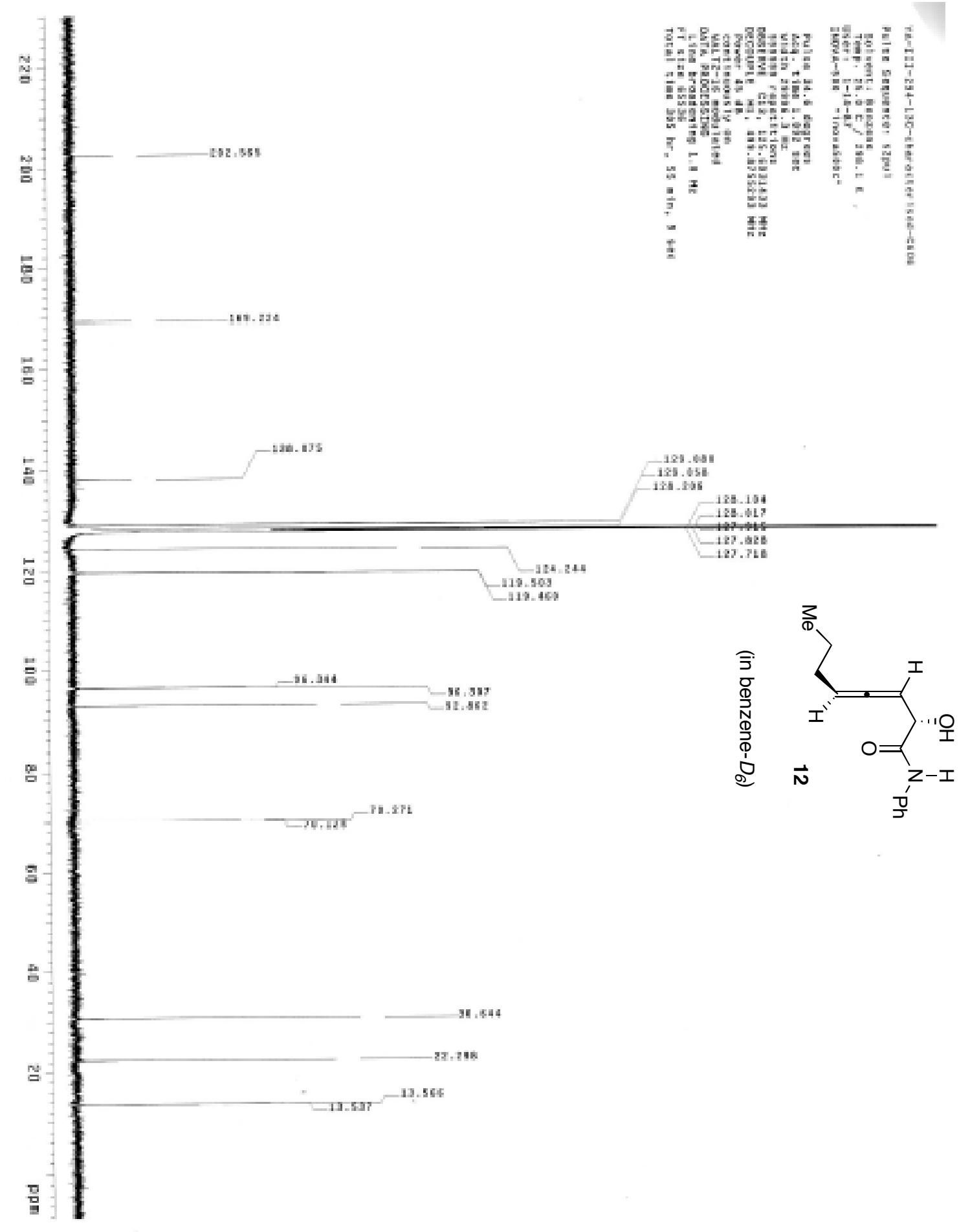




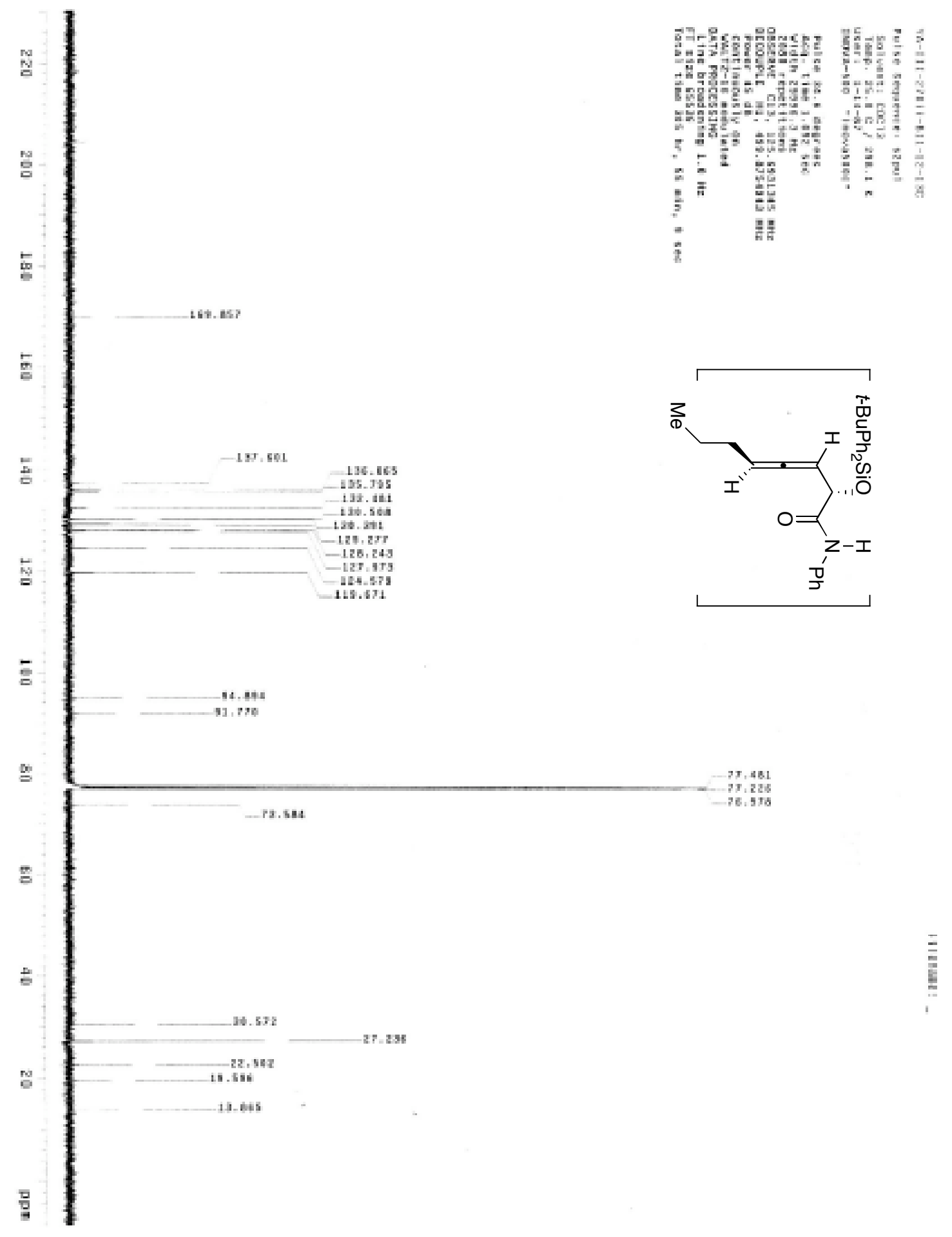




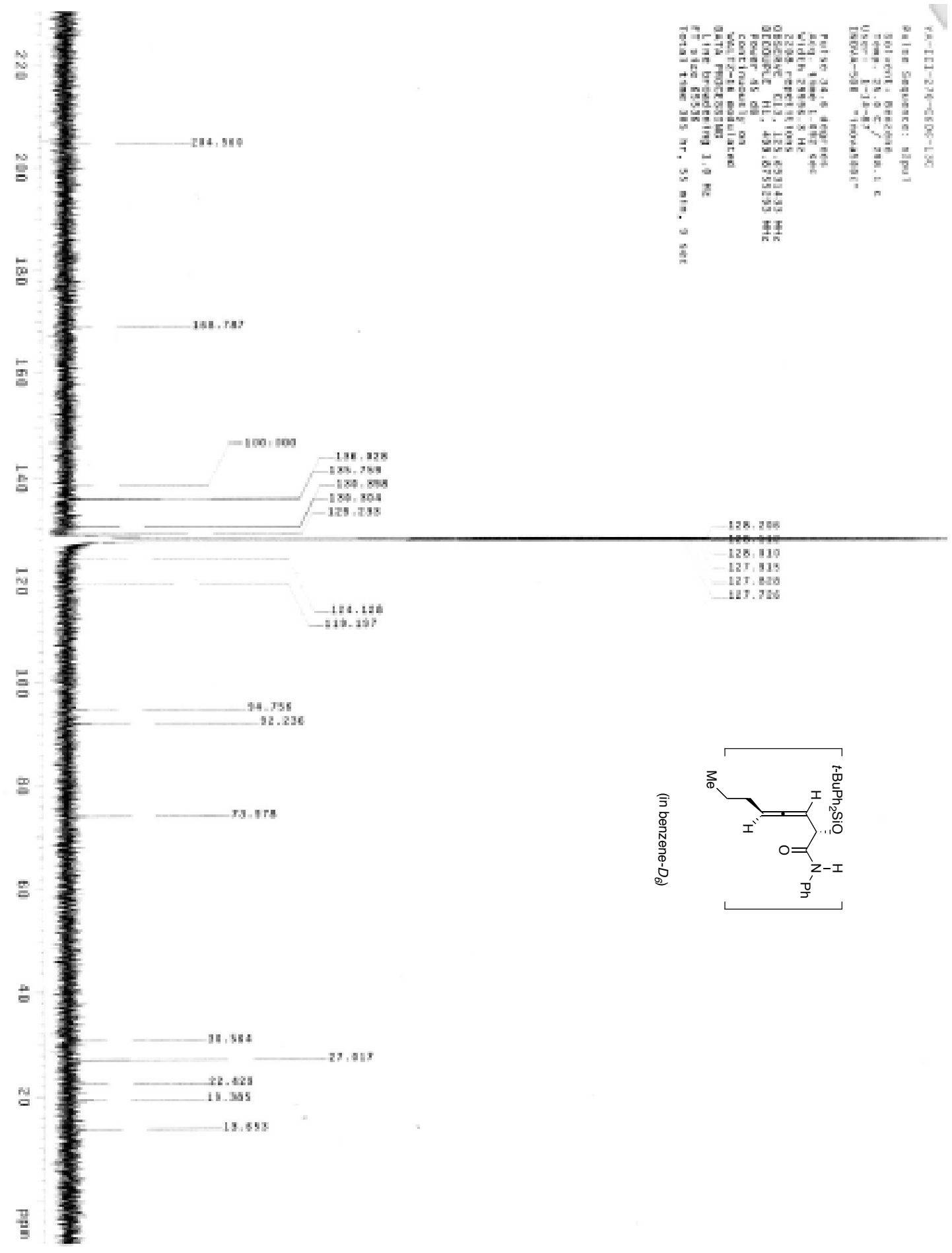




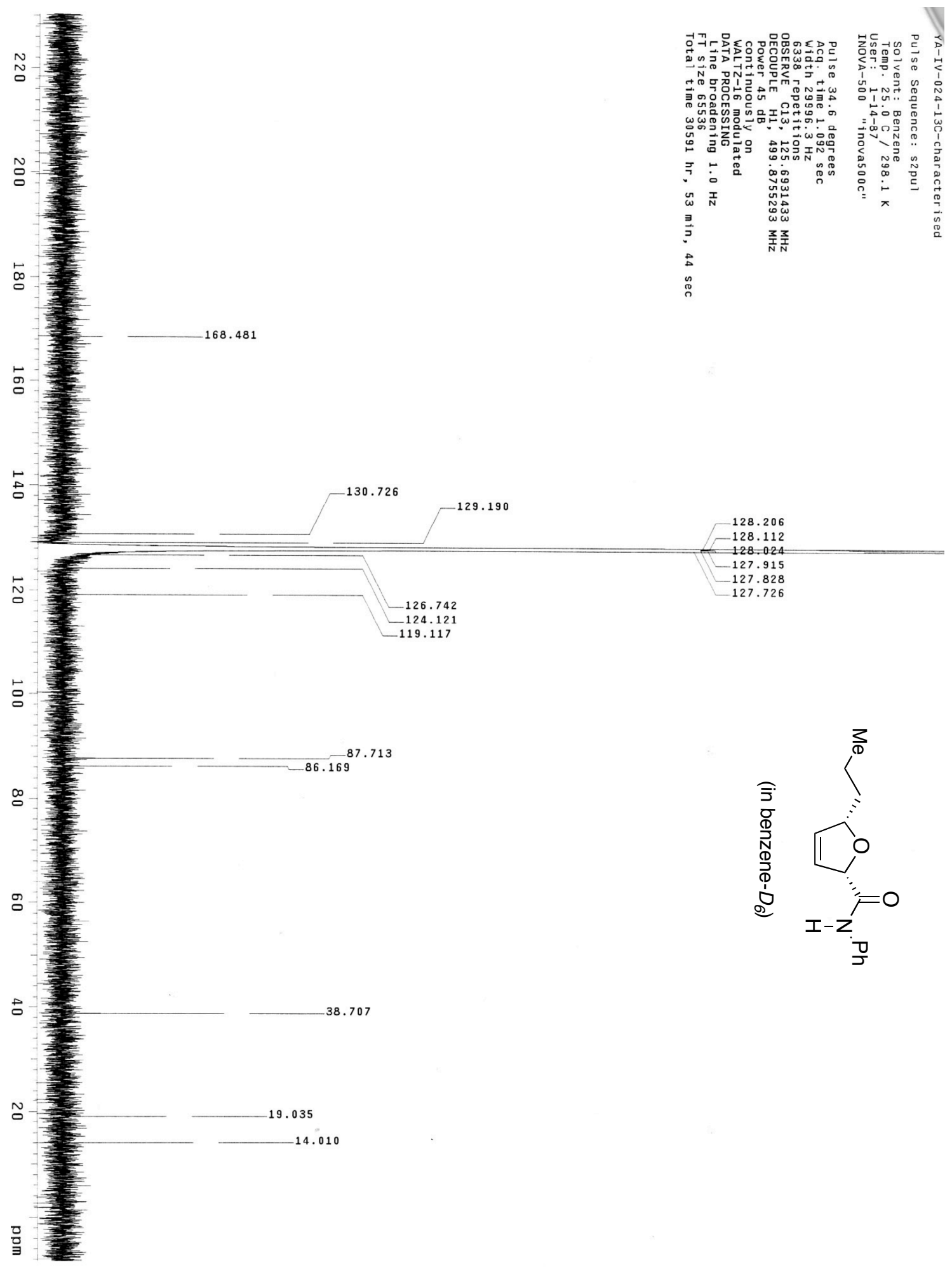




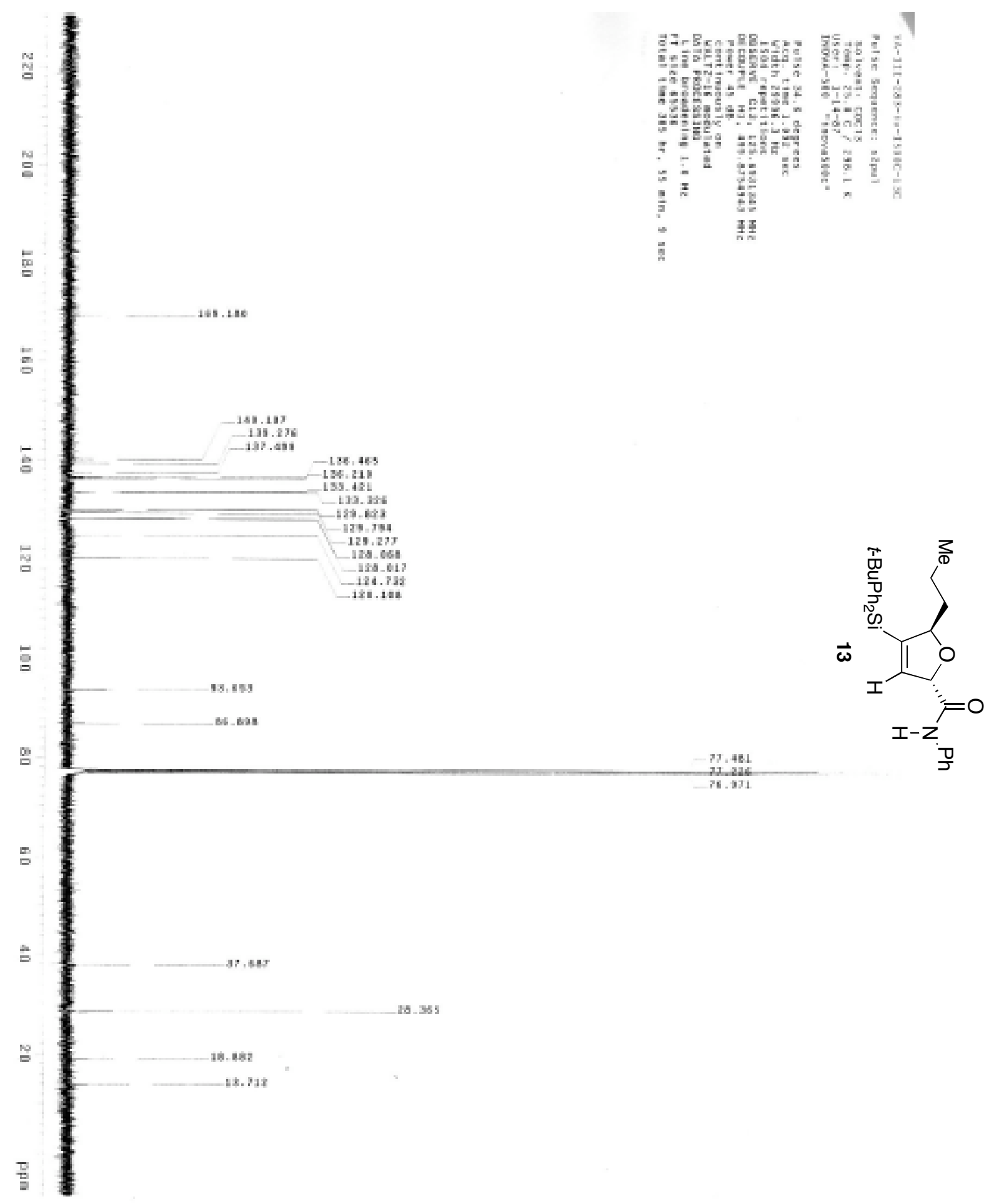




\section{ANALYTICAL HPLC TRACES}

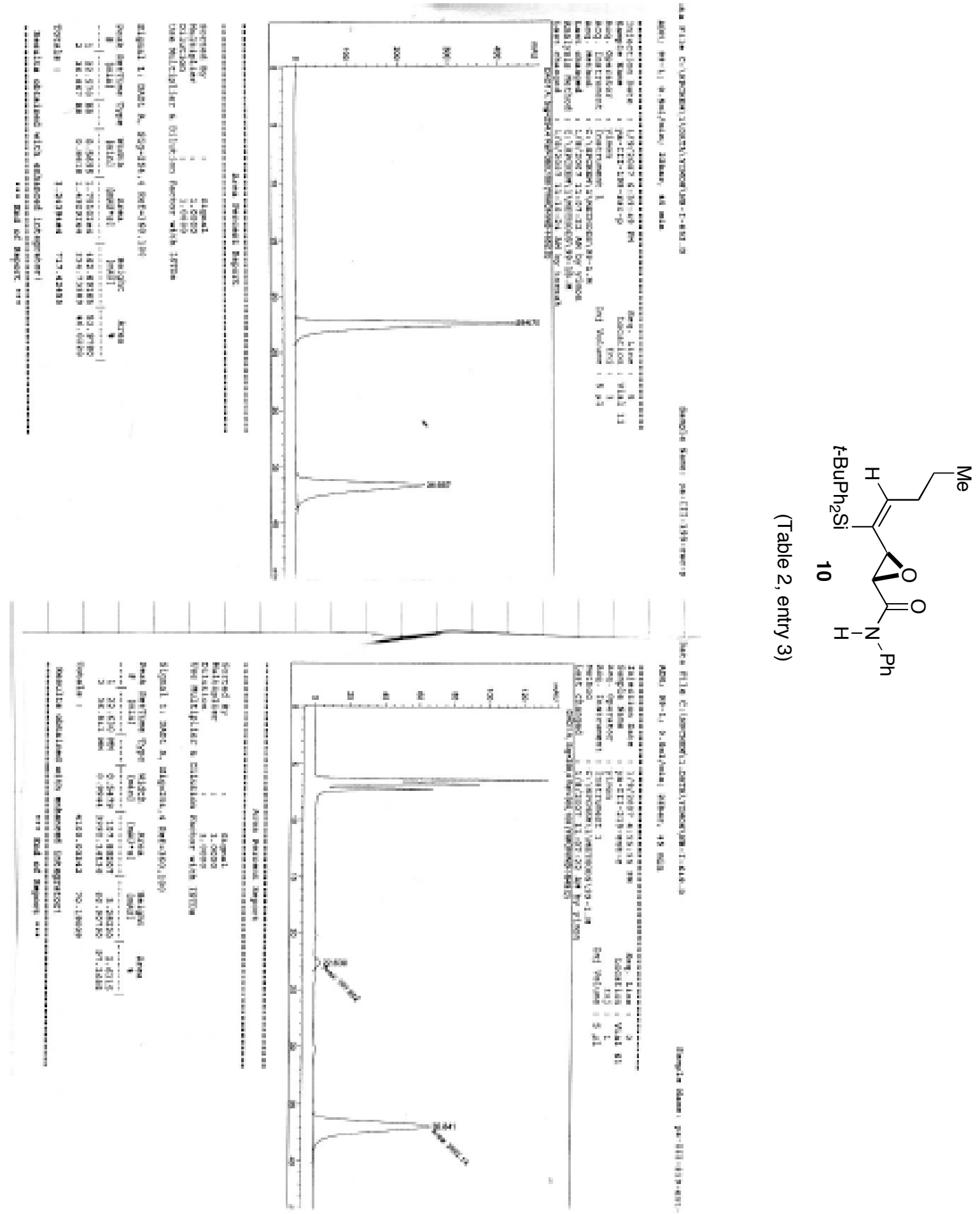




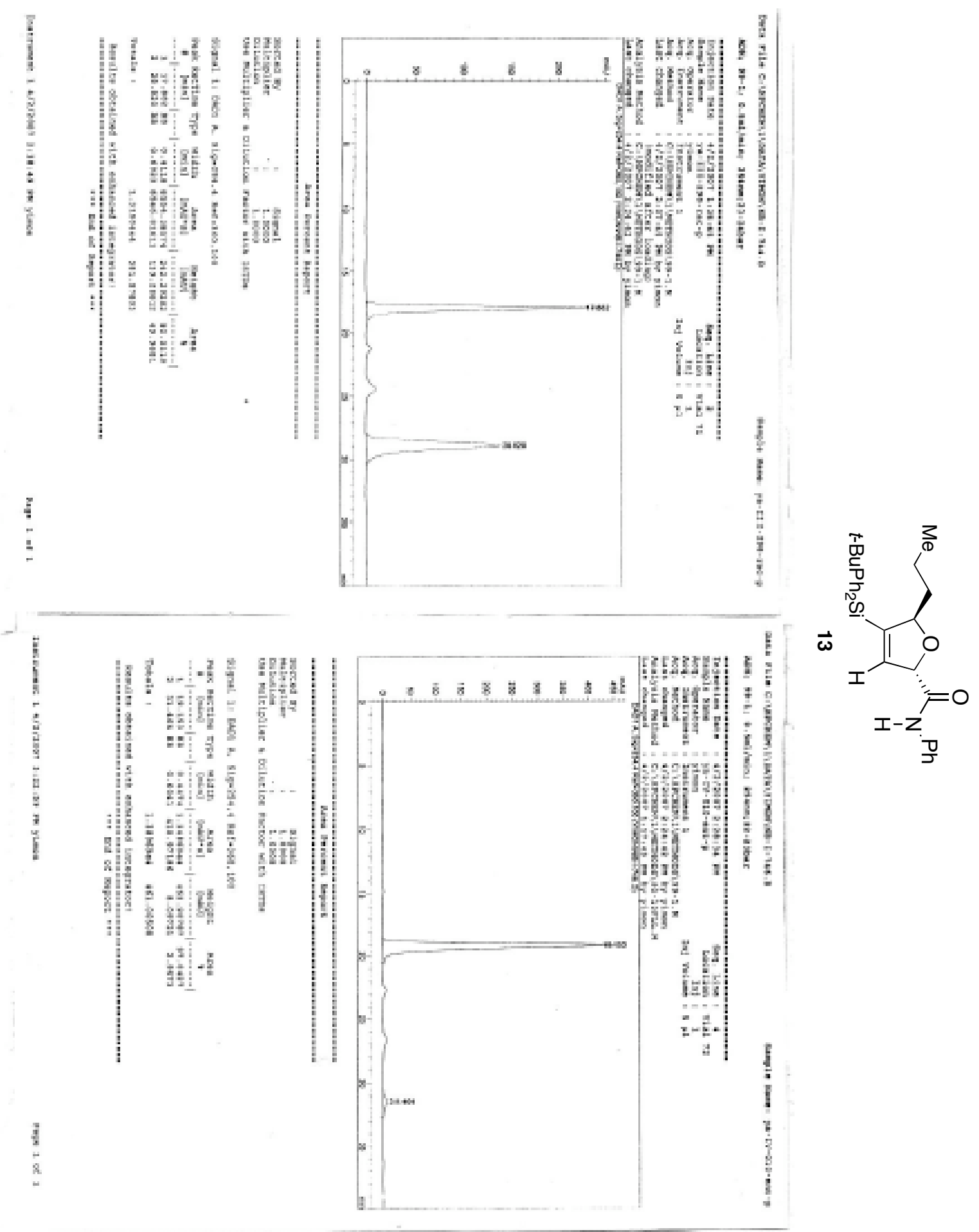




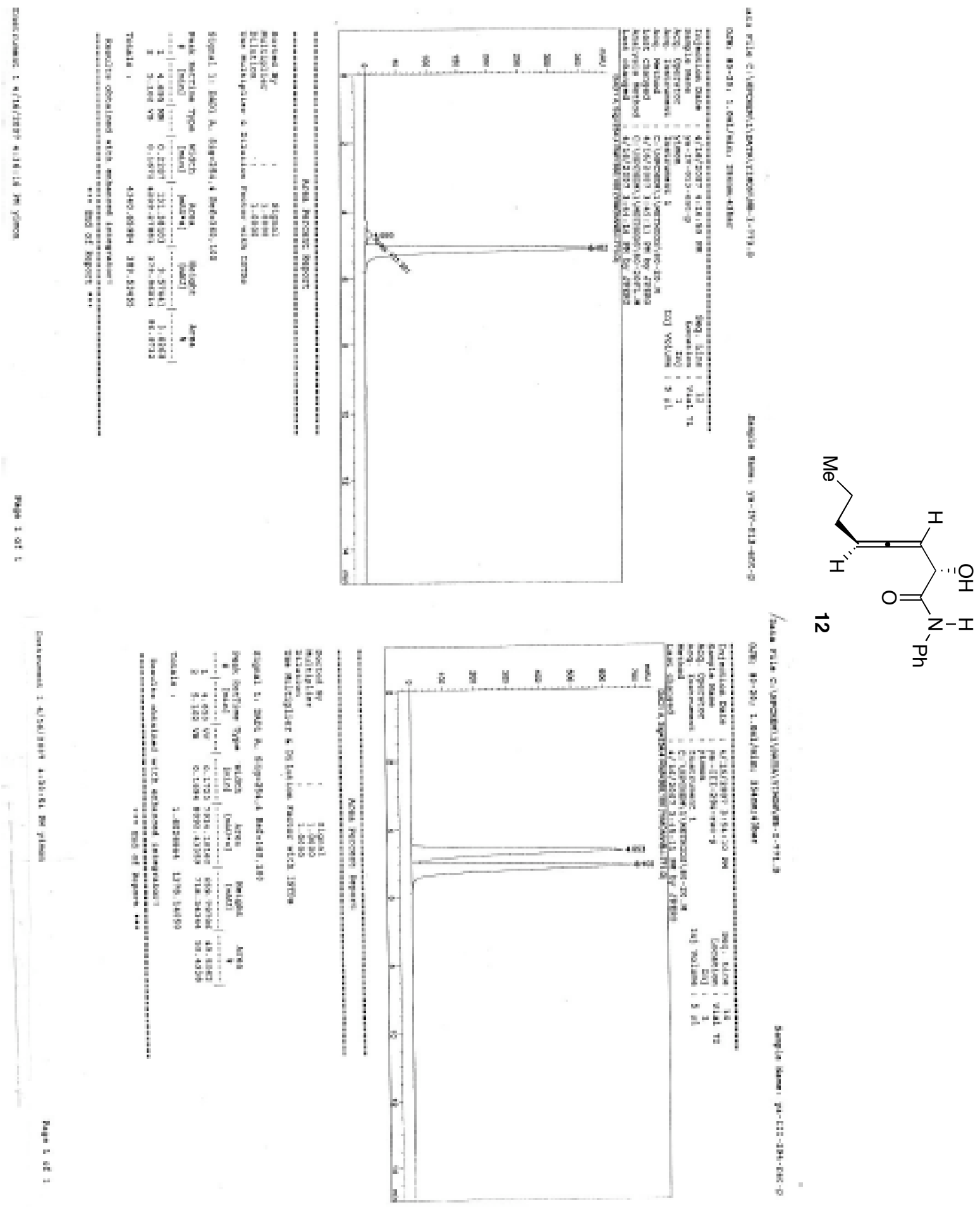




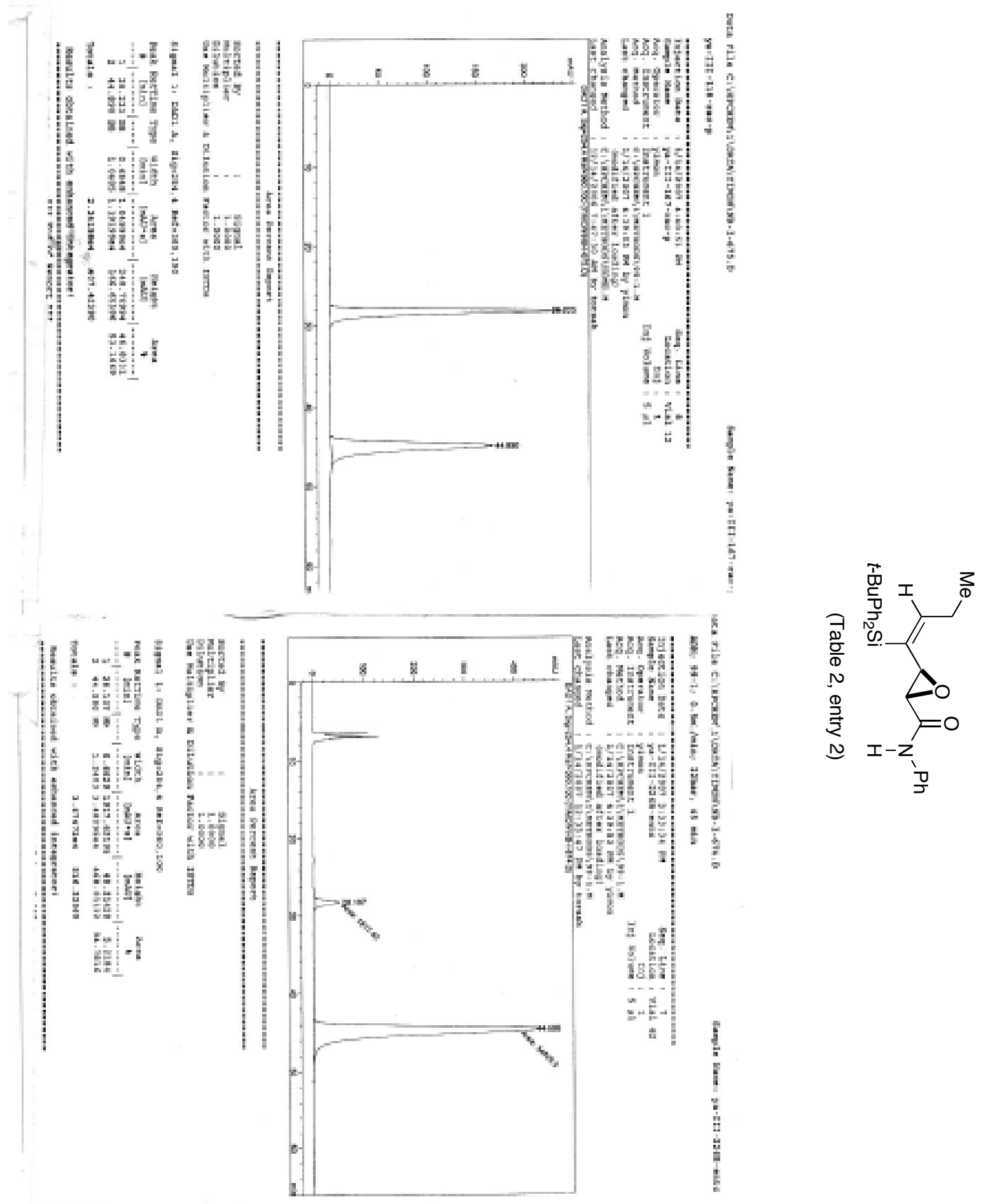




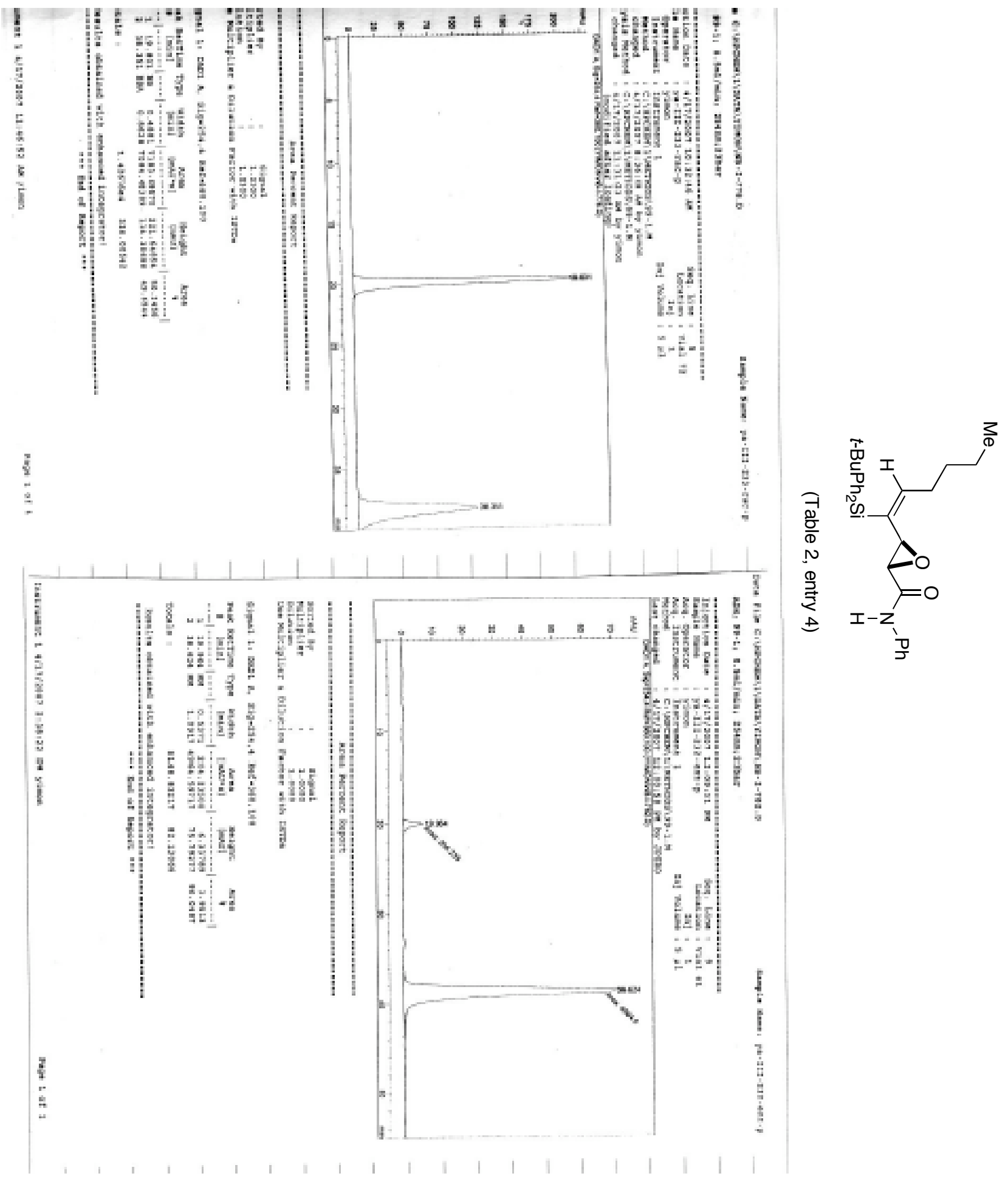



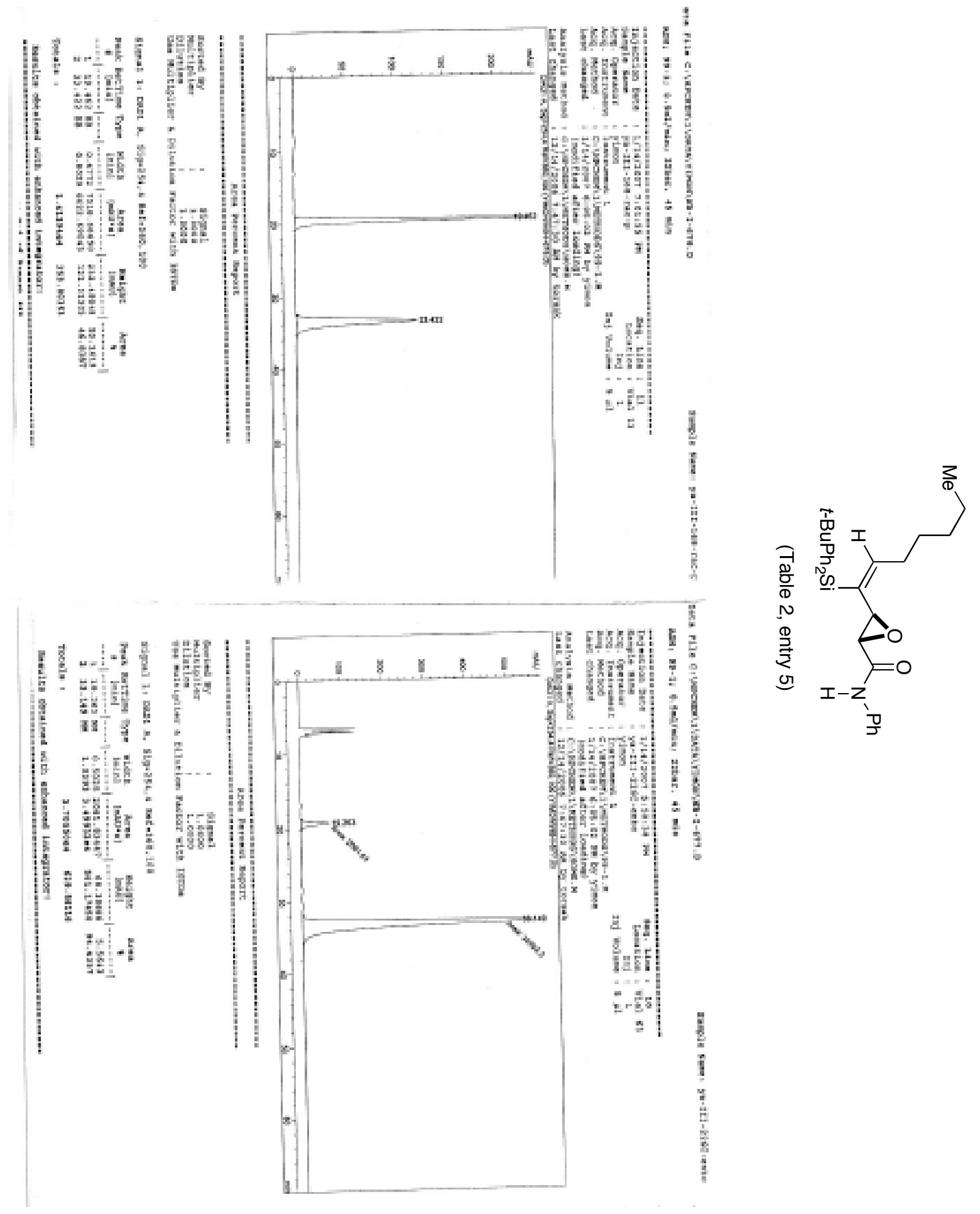


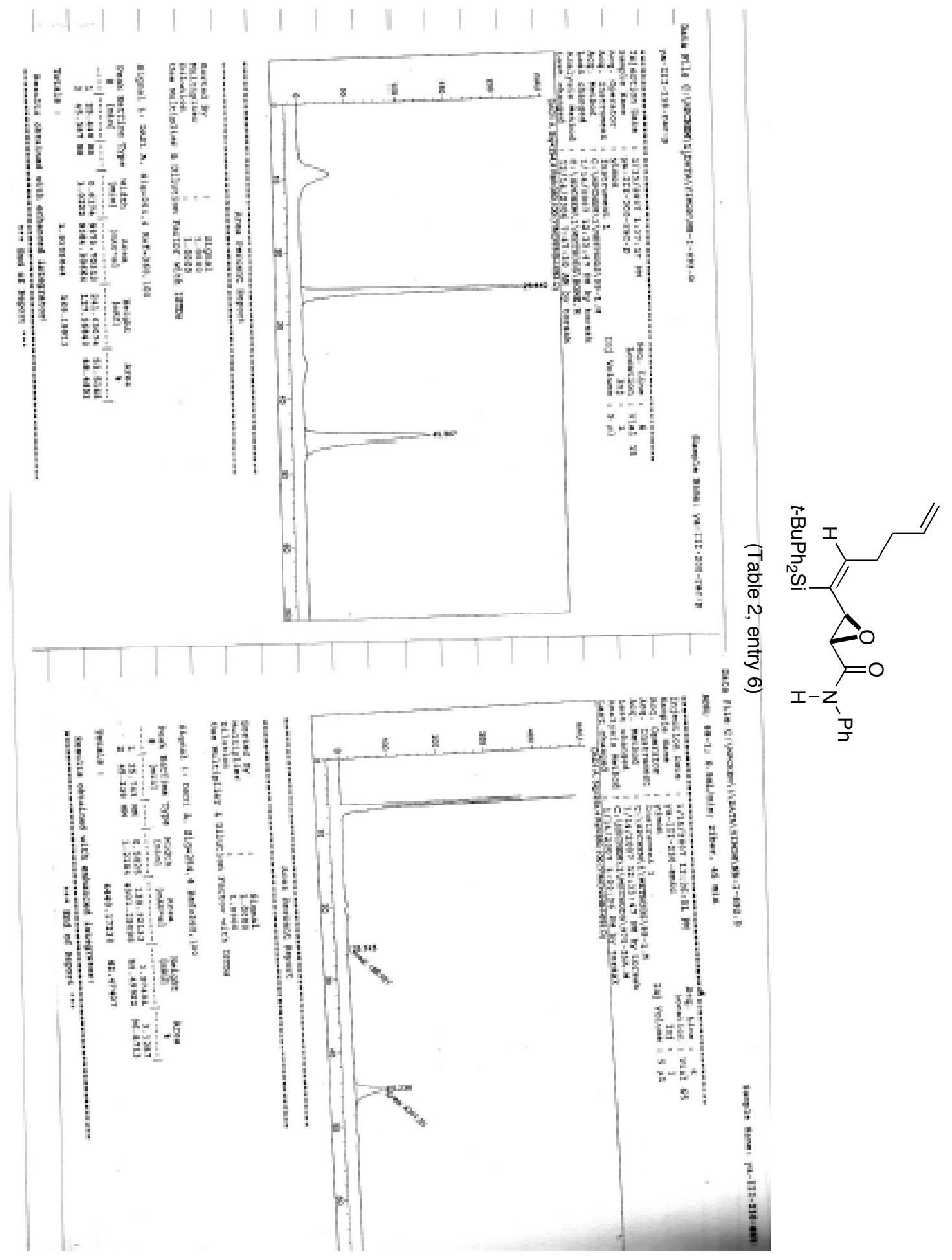



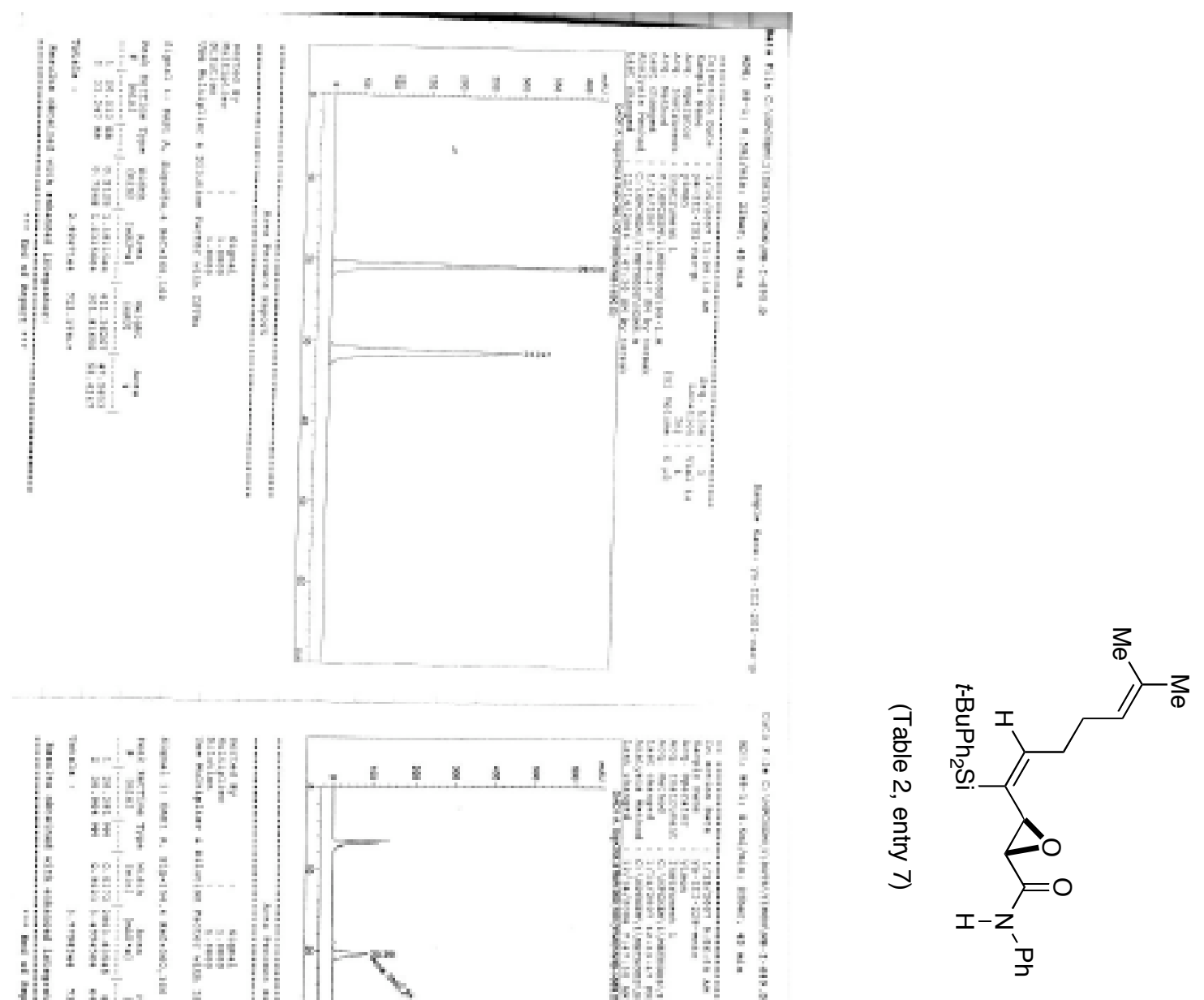


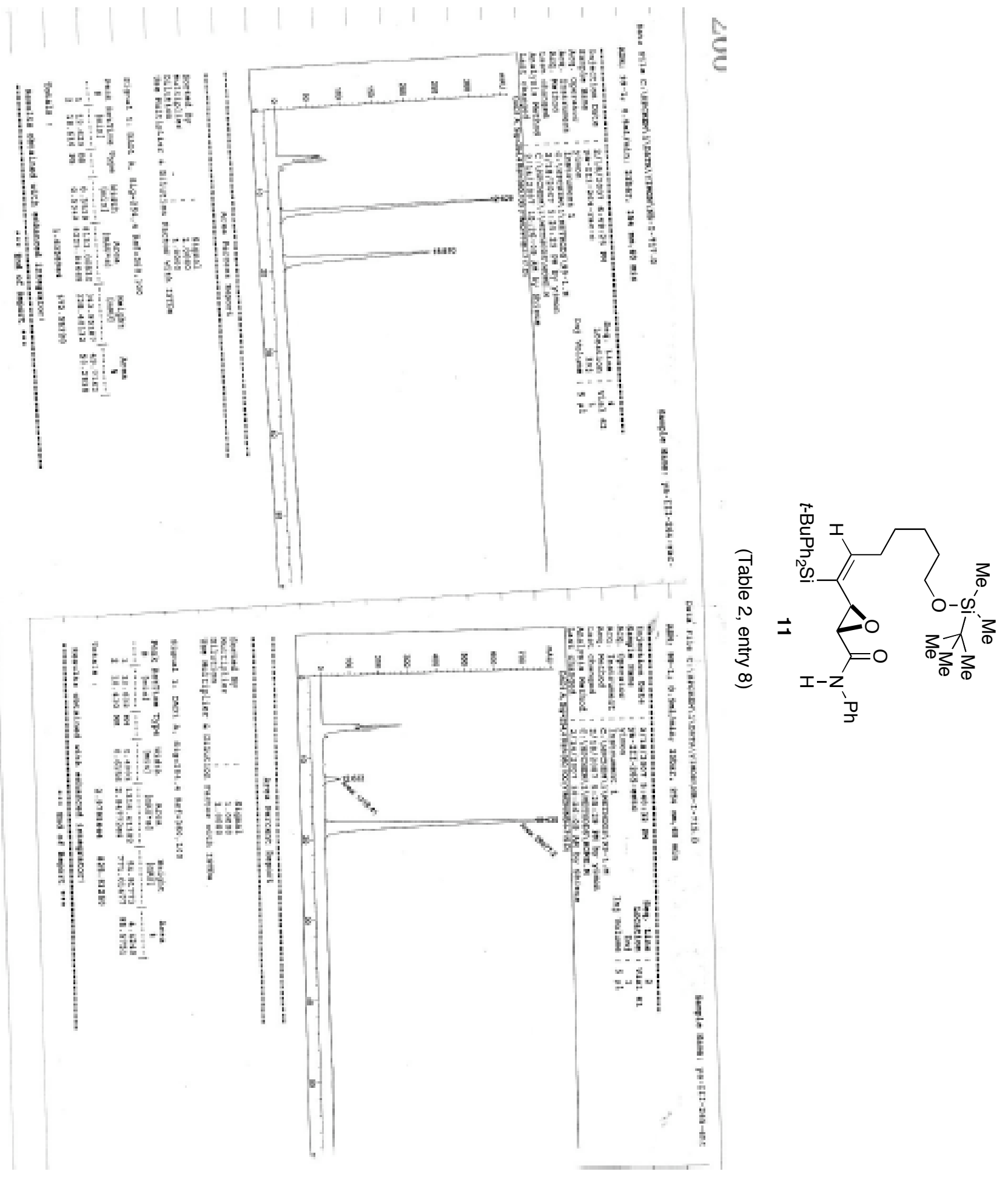


SINGLE CRYSTAL X-RAY DATA (see cif files for more information)

Table 1. Crystal data and structure refinement for ya37.

Identification code

Empirical formula

Formula weight

Temperature

Wavelength

Crystal system

Space group

Unit cell dimensions

Volume

Z

Density (calculated)

Absorption coefficient

$\mathrm{F}(000)$

Crystal size

Theta range for data collection

Index ranges

Reflections collected

Independent reflections

Completeness to theta $=27.90^{\circ}$

Absorption correction

Refinement method

Data / restraints / parameters

Goodness-of-fit on $\mathrm{F}^{2}$

Final R indices [I $>2 \operatorname{sigma}(\mathrm{I})]$

$\mathrm{R}$ indices (all data)

Largest diff. peak and hole ya37

C25 H33 N O2 Si

407.61

293(2) K

$0.71073 \AA$

Monoclinic

$\mathrm{P} 2(1) / \mathrm{c}$

$\mathrm{a}=14.094(2) \AA$

$\mathrm{b}=18.133(3) \AA$

$c=10.0075(14) \AA$

2423.2(6) $\AA^{3}$

4

$1.117 \mathrm{Mg} / \mathrm{m}^{3}$

$0.116 \mathrm{~mm}^{-1}$

880

$0.05 \times 0.04 \times 0.02 \mathrm{~mm}^{3}$

1.52 to $27.90^{\circ}$.

$-18<=\mathrm{h}<=18,-23<=\mathrm{k}<=17,-13<=\mathrm{l}<=11$

15997

$5731[\mathrm{R}(\mathrm{int})=0.1143]$

$99.0 \%$

None

Full-matrix least-squares on $\mathrm{F}^{2}$

$5731 / 0 / 394$

0.664

$\mathrm{R} 1=0.0468, \mathrm{wR} 2=0.0553$

$\mathrm{R} 1=0.2004, \mathrm{wR} 2=0.0775$

0.195 and -0.188 e. $\AA^{-3}$ $\mathrm{a}=90^{\circ}$.

$\mathrm{b}=108.656(3)^{\circ}$.

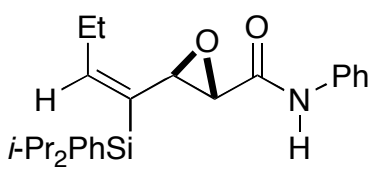

$( \pm)-4$

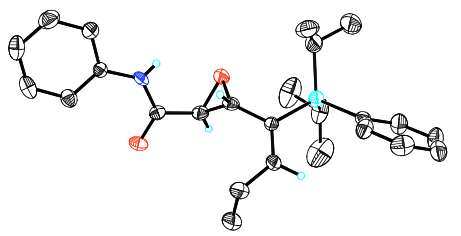

$\mathrm{g}=90^{\circ}$. 
Table 2. Atomic coordinates ( $\mathrm{x} 10^{4}$ ) and equivalent isotropic displacement parameters $\left(\AA^{2} \mathrm{x} 10^{3}\right)$ for ya37. $U(e q)$ is defined as one third of the trace of the orthogonalized $U^{i j}$ tensor.

\begin{tabular}{|c|c|c|c|c|}
\hline & $\mathrm{x}$ & $\mathrm{y}$ & $\mathrm{z}$ & $\mathrm{U}(\mathrm{eq})$ \\
\hline$C(1)$ & $14(2)$ & $2637(2)$ & $-1771(3)$ & $25(1)$ \\
\hline $\mathrm{C}(2)$ & $-1394(2)$ & $3317(2)$ & $-1335(3)$ & $23(1)$ \\
\hline$C(3)$ & $-1774(2)$ & 3531(2) & $-269(4)$ & $36(1)$ \\
\hline$C(4)$ & $-2658(2)$ & $3907(2)$ & $-590(4)$ & $45(1)$ \\
\hline$C(5)$ & $-3199(2)$ & 4088(2) & $-1963(4)$ & $47(1)$ \\
\hline$C(6)$ & $-2823(3)$ & $3869(2)$ & $-3019(4)$ & $43(1)$ \\
\hline$C(7)$ & $-1936(2)$ & $3486(2)$ & $-2738(3)$ & $33(1)$ \\
\hline$C(8)$ & $948(2)$ & $2226(2)$ & $-1057(3)$ & $28(1)$ \\
\hline $\mathrm{C}(9)$ & $963(2)$ & 1596(2) & $-113(3)$ & $26(1)$ \\
\hline$C(10)$ & $1637(2)$ & $943(2)$ & $-9(3)$ & $26(1)$ \\
\hline$C(11)$ & 1374(2) & $462(2)$ & $-1076(3)$ & $34(1)$ \\
\hline$C(12)$ & $504(3)$ & 492(2) & $-2417(4)$ & $44(1)$ \\
\hline$C(13)$ & $875(4)$ & $636(3)$ & $-3660(4)$ & $65(1)$ \\
\hline$C(14)$ & $3915(2)$ & 1193(2) & 1063(5) & $50(1)$ \\
\hline$C(15)$ & 4015(4) & $893(3)$ & $-301(6)$ & $86(2)$ \\
\hline$C(16)$ & 3932(4) & 2039(3) & 1030(7) & $78(2)$ \\
\hline $\mathrm{C}(17)$ & 2717(3) & $1235(2)$ & $3146(4)$ & $49(1)$ \\
\hline$C(18)$ & 1792(4) & $973(3)$ & $3466(5)$ & $62(1)$ \\
\hline$C(19)$ & $3660(5)$ & 1089(3) & 4405(5) & $84(2)$ \\
\hline$C(20)$ & 2994(2) & $-219(2)$ & 1766(3) & $25(1)$ \\
\hline$C(21)$ & 3940(3) & $-544(2)$ & 2208(3) & $40(1)$ \\
\hline$C(22)$ & 4076(3) & $-1295(2)$ & 2422(3) & $42(1)$ \\
\hline$C(23)$ & $3262(3)$ & $-1752(2)$ & 2168(3) & $42(1)$ \\
\hline$C(24)$ & 2321(3) & $-1448(2)$ & 1761(4) & $43(1)$ \\
\hline$C(25)$ & 2192(2) & $-694(2)$ & 1563(3) & $36(1)$ \\
\hline $\mathrm{N}(1)$ & $-478(2)$ & 2935(2) & $-953(3)$ & $28(1)$ \\
\hline $\mathrm{O}(1)$ & $-288(1)$ & 2662(1) & $-3080(2)$ & $38(1)$ \\
\hline $\mathrm{O}(2)$ & 1410(1) & 2298(1) & $441(2)$ & $34(1)$ \\
\hline $\operatorname{Si}(01)$ & 2814(1) & $807(1)$ & 1487(1) & $31(1)$ \\
\hline
\end{tabular}


Table 3. Bond lengths $[\AA ̊ \AA]$ and angles $\left[{ }^{\circ}\right]$ for ya37.

\begin{tabular}{|c|c|}
\hline $\mathrm{C}(1)-\mathrm{O}(1)$ & $1.242(3)$ \\
\hline $\mathrm{C}(1)-\mathrm{N}(1)$ & $1.344(3)$ \\
\hline$C(1)-C(8)$ & $1.482(4)$ \\
\hline $\mathrm{C}(2)-\mathrm{C}(3)$ & $1.393(4)$ \\
\hline$C(2)-C(7)$ & $1.401(4)$ \\
\hline $\mathrm{C}(2)-\mathrm{N}(1)$ & $1.406(3)$ \\
\hline$C(3)-C(4)$ & $1.366(4)$ \\
\hline$C(4)-C(5)$ & $1.382(4)$ \\
\hline$C(5)-C(6)$ & $1.384(4)$ \\
\hline$C(6)-C(7)$ & $1.378(4)$ \\
\hline $\mathrm{C}(8)-\mathrm{O}(2)$ & $1.436(3)$ \\
\hline $\mathrm{C}(8)-\mathrm{C}(9)$ & $1.478(4)$ \\
\hline $\mathrm{C}(9)-\mathrm{O}(2)$ & $1.450(3)$ \\
\hline$C(9)-C(10)$ & $1.500(4)$ \\
\hline $\mathrm{C}(10)-\mathrm{C}(11)$ & $1.337(4)$ \\
\hline $\mathrm{C}(10)-\mathrm{Si}(01)$ & $1.861(3)$ \\
\hline $\mathrm{C}(11)-\mathrm{C}(12)$ & $1.501(4)$ \\
\hline $\mathrm{C}(12)-\mathrm{C}(13)$ & $1.518(5)$ \\
\hline$C(14)-C(15)$ & $1.516(6)$ \\
\hline$C(14)-C(16)$ & $1.535(5)$ \\
\hline $\mathrm{C}(14)-\mathrm{Si}(01)$ & $1.872(3)$ \\
\hline $\mathrm{C}(17)-\mathrm{C}(18)$ & $1.516(6)$ \\
\hline $\mathrm{C}(17)-\mathrm{C}(19)$ & $1.534(5)$ \\
\hline $\mathrm{C}(17)-\mathrm{Si}(01)$ & $1.877(4)$ \\
\hline$C(20)-C(25)$ & $1.384(4)$ \\
\hline $\mathrm{C}(20)-\mathrm{C}(21)$ & $1.394(4)$ \\
\hline $\mathrm{C}(20)-\mathrm{Si}(01)$ & $1.885(3)$ \\
\hline$C(21)-C(22)$ & $1.381(4)$ \\
\hline$C(22)-C(23)$ & $1.372(4)$ \\
\hline$C(23)-C(24)$ & $1.372(4)$ \\
\hline$C(24)-C(25)$ & $1.385(4)$ \\
\hline $\mathrm{O}(1)-\mathrm{C}(1)-\mathrm{N}(1)$ & $123.9(3)$ \\
\hline $\mathrm{O}(1)-\mathrm{C}(1)-\mathrm{C}(8)$ & $118.6(3)$ \\
\hline
\end{tabular}




\begin{tabular}{|c|c|}
\hline $\mathrm{N}(1)-\mathrm{C}(1)-\mathrm{C}(8)$ & $117.4(3)$ \\
\hline$C(3)-C(2)-C(7)$ & 119.1(3) \\
\hline $\mathrm{C}(3)-\mathrm{C}(2)-\mathrm{N}(1)$ & $118.2(3)$ \\
\hline $\mathrm{C}(7)-\mathrm{C}(2)-\mathrm{N}(1)$ & $122.7(3)$ \\
\hline $\mathrm{C}(4)-\mathrm{C}(3)-\mathrm{C}(2)$ & $120.3(3)$ \\
\hline $\mathrm{C}(3)-\mathrm{C}(4)-\mathrm{C}(5)$ & $121.6(4)$ \\
\hline$C(4)-C(5)-C(6)$ & $117.8(3)$ \\
\hline$C(7)-C(6)-C(5)$ & $122.3(3)$ \\
\hline$C(6)-C(7)-C(2)$ & $118.9(3)$ \\
\hline $\mathrm{O}(2)-\mathrm{C}(8)-\mathrm{C}(9)$ & $59.65(18)$ \\
\hline $\mathrm{O}(2)-\mathrm{C}(8)-\mathrm{C}(1)$ & 119.6(3) \\
\hline $\mathrm{C}(9)-\mathrm{C}(8)-\mathrm{C}(1)$ & $121.7(3)$ \\
\hline $\mathrm{O}(2)-\mathrm{C}(9)-\mathrm{C}(8)$ & $58.73(18)$ \\
\hline $\mathrm{O}(2)-\mathrm{C}(9)-\mathrm{C}(10)$ & $118.6(2)$ \\
\hline $\mathrm{C}(8)-\mathrm{C}(9)-\mathrm{C}(10)$ & $122.2(3)$ \\
\hline $\mathrm{C}(11)-\mathrm{C}(10)-\mathrm{C}(9)$ & $116.9(3)$ \\
\hline$C(11)-C(10)-S i(01)$ & $120.2(2)$ \\
\hline $\mathrm{C}(9)-\mathrm{C}(10)-\mathrm{Si}(01)$ & $122.9(2)$ \\
\hline $\mathrm{C}(10)-\mathrm{C}(11)-\mathrm{C}(12)$ & $128.9(3)$ \\
\hline $\mathrm{C}(11)-\mathrm{C}(12)-\mathrm{C}(13)$ & $110.0(3)$ \\
\hline $\mathrm{C}(15)-\mathrm{C}(14)-\mathrm{C}(16)$ & $109.4(4)$ \\
\hline$C(15)-C(14)-\operatorname{Si}(01)$ & 113.1(3) \\
\hline$C(16)-C(14)-S i(01)$ & $113.5(3)$ \\
\hline $\mathrm{C}(18)-\mathrm{C}(17)-\mathrm{C}(19)$ & $110.5(4)$ \\
\hline $\mathrm{C}(18)-\mathrm{C}(17)-\mathrm{Si}(01)$ & $111.6(3)$ \\
\hline $\mathrm{C}(19)-\mathrm{C}(17)-\mathrm{Si}(01)$ & $111.5(3)$ \\
\hline$C(25)-C(20)-C(21)$ & $115.8(3)$ \\
\hline$C(25)-C(20)-S i(01)$ & $121.8(2)$ \\
\hline$C(21)-C(20)-\operatorname{Si}(01)$ & $122.3(2)$ \\
\hline $\mathrm{C}(22)-\mathrm{C}(21)-\mathrm{C}(20)$ & $122.6(3)$ \\
\hline $\mathrm{C}(23)-\mathrm{C}(22)-\mathrm{C}(21)$ & $120.0(4)$ \\
\hline $\mathrm{C}(22)-\mathrm{C}(23)-\mathrm{C}(24)$ & $118.9(4)$ \\
\hline $\mathrm{C}(23)-\mathrm{C}(24)-\mathrm{C}(25)$ & $120.6(4)$ \\
\hline $\mathrm{C}(20)-\mathrm{C}(25)-\mathrm{C}(24)$ & $122.0(3)$ \\
\hline $\mathrm{C}(1)-\mathrm{N}(1)-\mathrm{C}(2)$ & $129.8(3)$ \\
\hline $\mathrm{C}(8)-\mathrm{O}(2)-\mathrm{C}(9)$ & $61.62(17)$ \\
\hline
\end{tabular}


C(10)-Si(01)-C(14)

C(10)-Si(01)-C(17)

C(14)-Si(01)-C(17)

C(10)-Si(01)-C(20)

C(14)-Si(01)-C(20)

C(17)-Si(01)-C(20)
110.97(15)

110.99(15)

110.23(19)

107.09(13)

108.65(15)

108.80(15)

Symmetry transformations used to generate equivalent atoms: 
Table 4. Anisotropic displacement parameters $\left(\AA^{2} \times 10^{3}\right)$ for ya37. The anisotropic displacement factor exponent takes the form: $-2 \mathrm{p}^{2}\left[\mathrm{~h}^{2} \mathrm{a}^{* 2} \mathrm{U}^{11}+\ldots+2 \mathrm{~h} \mathrm{k} \mathrm{a}^{*} \mathrm{~b}^{*} \mathrm{U}^{12}\right]$

\begin{tabular}{|c|c|c|c|c|c|c|}
\hline & $\mathrm{U}^{11}$ & $\mathrm{U}^{22}$ & $\mathrm{U}^{33}$ & $\mathrm{U}^{23}$ & $\mathrm{U}^{13}$ & $\mathrm{U}^{12}$ \\
\hline $\mathrm{C}(1)$ & $27(2)$ & $25(2)$ & $27(2)$ & $-1(2)$ & $14(2)$ & $-3(2)$ \\
\hline$C(2)$ & $26(2)$ & $18(2)$ & $24(2)$ & $3(2)$ & $7(2)$ & $0(2)$ \\
\hline$C(3)$ & $32(2)$ & $46(2)$ & $31(2)$ & $0(2)$ & $11(2)$ & $8(2)$ \\
\hline$C(4)$ & $41(2)$ & $52(3)$ & $47(3)$ & $-7(2)$ & $22(2)$ & $3(2)$ \\
\hline$C(5)$ & $30(2)$ & $48(3)$ & $58(3)$ & $7(2)$ & $6(2)$ & $8(2)$ \\
\hline$C(6)$ & $36(2)$ & $48(3)$ & $38(2)$ & $13(2)$ & $-1(2)$ & $0(2)$ \\
\hline$C(7)$ & $32(2)$ & $37(2)$ & $30(2)$ & $6(2)$ & $10(2)$ & $0(2)$ \\
\hline$C(8)$ & $27(2)$ & $34(2)$ & $27(2)$ & $-2(2)$ & $14(2)$ & $-1(2)$ \\
\hline $\mathrm{C}(9)$ & $26(2)$ & $33(2)$ & $21(2)$ & $-2(2)$ & $11(2)$ & $-2(2)$ \\
\hline$C(10)$ & $33(2)$ & $27(2)$ & $20(2)$ & $3(2)$ & $13(1)$ & $7(2)$ \\
\hline $\mathrm{C}(11)$ & $38(2)$ & $41(2)$ & $23(2)$ & $5(2)$ & $11(2)$ & $13(2)$ \\
\hline$C(12)$ & $43(2)$ & $52(3)$ & $33(2)$ & $-8(2)$ & $6(2)$ & $8(2)$ \\
\hline$C(13)$ & 74(3) & $83(4)$ & $32(3)$ & $-5(3)$ & $9(2)$ & $35(3)$ \\
\hline$C(14)$ & $28(2)$ & $35(3)$ & $85(3)$ & $8(2)$ & $15(2)$ & $-4(2)$ \\
\hline$C(15)$ & $74(4)$ & $83(5)$ & $132(5)$ & $10(4)$ & $76(4)$ & $1(4)$ \\
\hline$C(16)$ & $56(3)$ & 41(3) & $141(6)$ & $10(4)$ & $38(4)$ & $-11(3)$ \\
\hline$C(17)$ & $66(3)$ & $30(3)$ & $40(2)$ & $-3(2)$ & $3(2)$ & $9(2)$ \\
\hline$C(18)$ & $105(4)$ & $48(3)$ & $45(3)$ & $-1(3)$ & $38(3)$ & $18(3)$ \\
\hline$C(19)$ & $111(5)$ & $68(4)$ & $44(3)$ & $-18(3)$ & $-15(3)$ & $28(4)$ \\
\hline$C(20)$ & $27(2)$ & $25(2)$ & $23(2)$ & $-2(2)$ & $6(2)$ & $-3(2)$ \\
\hline$C(21)$ & $34(2)$ & $33(3)$ & $46(2)$ & $1(2)$ & $4(2)$ & $-2(2)$ \\
\hline$C(22)$ & $31(2)$ & $35(3)$ & $53(2)$ & $6(2)$ & $4(2)$ & $6(2)$ \\
\hline$C(23)$ & $50(3)$ & $34(3)$ & $37(2)$ & $7(2)$ & $6(2)$ & $5(2)$ \\
\hline$C(24)$ & $46(3)$ & $36(3)$ & $50(2)$ & $-6(2)$ & $20(2)$ & $-16(2)$ \\
\hline$C(25)$ & $36(2)$ & $32(2)$ & $42(2)$ & $4(2)$ & $16(2)$ & $6(2)$ \\
\hline $\mathrm{N}(1)$ & $31(2)$ & $36(2)$ & $14(2)$ & $4(2)$ & $4(2)$ & $3(1)$ \\
\hline $\mathrm{O}(1)$ & $48(1)$ & $48(2)$ & $20(1)$ & $0(1)$ & $15(1)$ & $4(1)$ \\
\hline $\mathrm{O}(2)$ & $37(1)$ & $32(1)$ & $28(1)$ & $-5(1)$ & 1(1) & $2(1)$ \\
\hline $\operatorname{Si}(01)$ & $29(1)$ & $26(1)$ & $34(1)$ & $-1(1)$ & $4(1)$ & $0(1)$ \\
\hline
\end{tabular}


Table 5. Hydrogen coordinates ( $\left.\mathrm{x} 10^{4}\right)$ and isotropic displacement parameters $\left(\AA^{2} \mathrm{x} 10^{3}\right)$ for ya37.

\begin{tabular}{|c|c|c|c|c|}
\hline & $\mathrm{X}$ & $\mathrm{y}$ & $\mathrm{z}$ & $\mathrm{U}(\mathrm{eq})$ \\
\hline $\mathrm{H}(1)$ & $-204(18)$ & $2870(16)$ & $-230(20)$ & $18(10)$ \\
\hline $\mathrm{H}(3)$ & $-1355(18)$ & $3426(16)$ & $650(30)$ & $53(11)$ \\
\hline $\mathrm{H}(4)$ & $-2893(16)$ & $4079(15)$ & $160(20)$ & $35(9)$ \\
\hline $\mathrm{H}(5)$ & $-3810(19)$ & $4400(17)$ & $-2250(30)$ & $68(12)$ \\
\hline $\mathrm{H}(6)$ & $-3214(18)$ & $4014(16)$ & $-3880(30)$ & $52(11)$ \\
\hline $\mathrm{H}(7)$ & $-1600(20)$ & $3318(16)$ & $-3370(30)$ & $68(12)$ \\
\hline $\mathrm{H}(8)$ & $1434(17)$ & $2215(14)$ & $-1490(30)$ & $43(10)$ \\
\hline $\mathrm{H}(9)$ & $340(14)$ & $1515(11)$ & $53(19)$ & $1(6)$ \\
\hline $\mathrm{H}(11)$ & $1862(17)$ & $48(13)$ & $-1010(20)$ & $31(8)$ \\
\hline $\mathrm{H}(14)$ & $4530(19)$ & $1038(17)$ & $1900(30)$ & $72(12)$ \\
\hline $\mathrm{H}(17)$ & $2660(20)$ & $1729(16)$ & $3000(30)$ & $50(11)$ \\
\hline $\mathrm{H}(21)$ & $4507(17)$ & $-243(14)$ & $2330(30)$ & $34(9)$ \\
\hline $\mathrm{H}(22)$ & $4738(16)$ & $-1488(13)$ & $2660(20)$ & $24(8)$ \\
\hline $\mathrm{H}(23)$ & $3435(19)$ & $-2266(16)$ & $2480(30)$ & $57(11)$ \\
\hline $\mathrm{H}(24)$ & 1783(18) & $-1752(15)$ & $1600(30)$ & $40(10)$ \\
\hline $\mathrm{H}(25)$ & $1513(16)$ & $-506(13)$ & $1260(20)$ & $22(8)$ \\
\hline $\mathrm{H}(12 \mathrm{~A})$ & $2(18)$ & $870(15)$ & $-2380(30)$ & $48(10)$ \\
\hline $\mathrm{H}(13 \mathrm{~A})$ & $320(20)$ & $690(20)$ & $-4460(30)$ & $99(15)$ \\
\hline $\mathrm{H}(15 \mathrm{~A})$ & $4060(20)$ & $320(20)$ & $-310(40)$ & $97(16)$ \\
\hline $\mathrm{H}(16 \mathrm{~A})$ & $3260(20)$ & $2230(20)$ & $250(40)$ & $116(16)$ \\
\hline $\mathrm{H}(18 \mathrm{~A})$ & $1760(20)$ & $1216(17)$ & $4190(30)$ & $51(12)$ \\
\hline $\mathrm{H}(19 \mathrm{~A})$ & $3570(20)$ & $1282(17)$ & $5150(30)$ & $46(12)$ \\
\hline $\mathrm{H}(12 \mathrm{~B})$ & $100(20)$ & $23(15)$ & $-2570(30)$ & $55(11)$ \\
\hline $\mathrm{H}(13 \mathrm{~B})$ & 1301(19) & $247(16)$ & $-3770(30)$ & $39(10)$ \\
\hline $\mathrm{H}(15 \mathrm{~B})$ & $4660(20)$ & $1058(16)$ & $-410(30)$ & $61(11)$ \\
\hline $\mathrm{H}(16 \mathrm{~B})$ & $4490(20)$ & $2176(18)$ & $820(30)$ & $59(12)$ \\
\hline $\mathrm{H}(18 \mathrm{~B})$ & $1750(20)$ & $450(16)$ & $3600(30)$ & $47(11)$ \\
\hline $\mathrm{H}(19 \mathrm{~B})$ & $3810(20)$ & $608(18)$ & $4580(30)$ & $71(15)$ \\
\hline $\mathrm{H}(13 \mathrm{C})$ & $1360(30)$ & $1130(30)$ & $-3380(40)$ & $190(30)$ \\
\hline $\mathrm{H}(14 \mathrm{C})$ & $3960(30)$ & $2270(20)$ & $1870(30)$ & $85(18)$ \\
\hline
\end{tabular}




$\begin{array}{rrrrr}\mathrm{H}(15 \mathrm{C}) & 3472(19) & 1059(18) & -1060(30) & 51(13) \\ \mathrm{H}(18 \mathrm{C}) & 1030(20) & 1118(18) & 2630(30) & 88(13) \\ \mathrm{H}(19 \mathrm{C}) & 4330(20) & 1300(20) & 4150(30) & 103(17)\end{array}$


Table 6. Torsion angles $\left[{ }^{\circ}\right]$ for ya37.

\begin{tabular}{|c|c|}
\hline $\mathrm{C}(7)-\mathrm{C}(2)-\mathrm{C}(3)-\mathrm{C}(4)$ & $-0.6(5)$ \\
\hline $\mathrm{N}(1)-\mathrm{C}(2)-\mathrm{C}(3)-\mathrm{C}(4)$ & $179.5(3)$ \\
\hline$C(2)-C(3)-C(4)-C(5)$ & $-0.2(5)$ \\
\hline$C(3)-C(4)-C(5)-C(6)$ & $0.8(5)$ \\
\hline$C(4)-C(5)-C(6)-C(7)$ & $-0.5(5)$ \\
\hline$C(5)-C(6)-C(7)-C(2)$ & $-0.2(5)$ \\
\hline$C(3)-C(2)-C(7)-C(6)$ & $0.8(5)$ \\
\hline $\mathrm{N}(1)-\mathrm{C}(2)-\mathrm{C}(7)-\mathrm{C}(6)$ & $-179.3(3)$ \\
\hline $\mathrm{O}(1)-\mathrm{C}(1)-\mathrm{C}(8)-\mathrm{O}(2)$ & $168.8(3)$ \\
\hline $\mathrm{N}(1)-\mathrm{C}(1)-\mathrm{C}(8)-\mathrm{O}(2)$ & $-13.9(4)$ \\
\hline $\mathrm{O}(1)-\mathrm{C}(1)-\mathrm{C}(8)-\mathrm{C}(9)$ & $-120.7(3)$ \\
\hline $\mathrm{N}(1)-\mathrm{C}(1)-\mathrm{C}(8)-\mathrm{C}(9)$ & $56.7(4)$ \\
\hline $\mathrm{C}(1)-\mathrm{C}(8)-\mathrm{C}(9)-\mathrm{O}(2)$ & $-108.1(3)$ \\
\hline $\mathrm{O}(2)-\mathrm{C}(8)-\mathrm{C}(9)-\mathrm{C}(10)$ & $-106.2(3)$ \\
\hline $\mathrm{C}(1)-\mathrm{C}(8)-\mathrm{C}(9)-\mathrm{C}(10)$ & $145.7(3)$ \\
\hline $\mathrm{O}(2)-\mathrm{C}(9)-\mathrm{C}(10)-\mathrm{C}(11)$ & $-144.6(3)$ \\
\hline $\mathrm{C}(8)-\mathrm{C}(9)-\mathrm{C}(10)-\mathrm{C}(11)$ & $-75.4(4)$ \\
\hline $\mathrm{O}(2)-\mathrm{C}(9)-\mathrm{C}(10)-\mathrm{Si}(01)$ & $33.4(3)$ \\
\hline $\mathrm{C}(8)-\mathrm{C}(9)-\mathrm{C}(10)-\mathrm{Si}(01)$ & $102.5(3)$ \\
\hline $\mathrm{C}(9)-\mathrm{C}(10)-\mathrm{C}(11)-\mathrm{C}(12)$ & $2.5(5)$ \\
\hline $\mathrm{Si}(01)-\mathrm{C}(10)-\mathrm{C}(11)-\mathrm{C}(12)$ & $-175.5(3)$ \\
\hline $\mathrm{C}(10)-\mathrm{C}(11)-\mathrm{C}(12)-\mathrm{C}(13)$ & $109.7(4)$ \\
\hline $\mathrm{C}(25)-\mathrm{C}(20)-\mathrm{C}(21)-\mathrm{C}(22)$ & $0.6(5)$ \\
\hline $\mathrm{Si}(01)-\mathrm{C}(20)-\mathrm{C}(21)-\mathrm{C}(22)$ & $179.6(3)$ \\
\hline $\mathrm{C}(20)-\mathrm{C}(21)-\mathrm{C}(22)-\mathrm{C}(23)$ & $1.5(6)$ \\
\hline $\mathrm{C}(21)-\mathrm{C}(22)-\mathrm{C}(23)-\mathrm{C}(24)$ & $-2.8(5)$ \\
\hline $\mathrm{C}(22)-\mathrm{C}(23)-\mathrm{C}(24)-\mathrm{C}(25)$ & $2.2(5)$ \\
\hline$C(21)-C(20)-C(25)-C(24)$ & $-1.2(5)$ \\
\hline $\mathrm{Si}(01)-\mathrm{C}(20)-\mathrm{C}(25)-\mathrm{C}(24)$ & 179.7(3) \\
\hline $\mathrm{C}(23)-\mathrm{C}(24)-\mathrm{C}(25)-\mathrm{C}(20)$ & $-0.1(5)$ \\
\hline $\mathrm{O}(1)-\mathrm{C}(1)-\mathrm{N}(1)-\mathrm{C}(2)$ & $0.7(5)$ \\
\hline $\mathrm{C}(8)-\mathrm{C}(1)-\mathrm{N}(1)-\mathrm{C}(2)$ & $-176.4(3)$ \\
\hline $\mathrm{C}(3)-\mathrm{C}(2)-\mathrm{N}(1)-\mathrm{C}(1)$ & $175.9(3)$ \\
\hline $\mathrm{C}(7)-\mathrm{C}(2)-\mathrm{N}(1)-\mathrm{C}(1)$ & $-4.0(5)$ \\
\hline
\end{tabular}




$\begin{array}{lc}\mathrm{C}(1)-\mathrm{C}(8)-\mathrm{O}(2)-\mathrm{C}(9) & 111.6(3) \\ \mathrm{C}(10)-\mathrm{C}(9)-\mathrm{O}(2)-\mathrm{C}(8) & 112.3(3) \\ \mathrm{C}(11)-\mathrm{C}(10)-\mathrm{Si}(01)-\mathrm{C}(14) & 84.4(3) \\ \mathrm{C}(9)-\mathrm{C}(10)-\mathrm{Si}(01)-\mathrm{C}(14) & -93.5(3) \\ \mathrm{C}(11)-\mathrm{C}(10)-\mathrm{Si}(01)-\mathrm{C}(17) & -152.7(3) \\ \mathrm{C}(9)-\mathrm{C}(10)-\mathrm{Si}(01)-\mathrm{C}(17) & 29.4(3) \\ \mathrm{C}(11)-\mathrm{C}(10)-\mathrm{Si}(01)-\mathrm{C}(20) & -34.1(3) \\ \mathrm{C}(9)-\mathrm{C}(10)-\mathrm{Si}(01)-\mathrm{C}(20) & 148.1(2) \\ \mathrm{C}(15)-\mathrm{C}(14)-\mathrm{Si}(01)-\mathrm{C}(10) & -54.3(4) \\ \mathrm{C}(16)-\mathrm{C}(14)-\mathrm{Si}(01)-\mathrm{C}(10) & 71.1(4) \\ \mathrm{C}(15)-\mathrm{C}(14)-\mathrm{Si}(01)-\mathrm{C}(17) & -177.7(3) \\ \mathrm{C}(16)-\mathrm{C}(14)-\mathrm{Si}(01)-\mathrm{C}(17) & -52.3(4) \\ \mathrm{C}(15)-\mathrm{C}(14)-\mathrm{Si}(01)-\mathrm{C}(20) & 63.2(4) \\ \mathrm{C}(16)-\mathrm{C}(14)-\mathrm{Si}(01)-\mathrm{C}(20) & -171.4(3) \\ \mathrm{C}(18)-\mathrm{C}(17)-\mathrm{Si}(01)-\mathrm{C}(10) & 52.7(3) \\ \mathrm{C}(19)-\mathrm{C}(17)-\mathrm{Si}(01)-\mathrm{C}(10) & 176.8(3) \\ \mathrm{C}(18)-\mathrm{C}(17)-\mathrm{Si}(01)-\mathrm{C}(14) & 176.1(3) \\ \mathrm{C}(19)-\mathrm{C}(17)-\mathrm{Si}(01)-\mathrm{C}(14) & -59.8(4) \\ \mathrm{C}(18)-\mathrm{C}(17)-\mathrm{Si}(01)-\mathrm{C}(20) & -64.9(3) \\ \mathrm{C}(19)-\mathrm{C}(17)-\mathrm{Si}(01)-\mathrm{C}(20) & 59.2(4) \\ \mathrm{C}(25)-\mathrm{C}(20)-\mathrm{Si}(01)-\mathrm{C}(10) & -35.0(3) \\ \mathrm{C}(21)-\mathrm{C}(20)-\mathrm{Si}(01)-\mathrm{C}(10) & 146.1(2) \\ \mathrm{C}(25)-\mathrm{C}(20)-\mathrm{Si}(01)-\mathrm{C}(14) & -154.9(3) \\ \mathrm{C}(21)-\mathrm{C}(20)-\mathrm{Si}(01)-\mathrm{C}(14) & 26.1(3) \\ \mathrm{C}(25)-\mathrm{C}(20)-\mathrm{Si}(01)-\mathrm{C}(17) & 85.1(3) \\ \mathrm{C}(21)-\mathrm{C}(20)-\mathrm{Si}(01)-\mathrm{C}(17) & -93.9(3)\end{array}$


Table 1. Crystal data and structure refinement for ya59sad.

Identification code

Empirical formula

Formula weight

Temperature

Wavelength

Crystal system

Space group

Unit cell dimensions

Volume

Z

Density (calculated)

Absorption coefficient

$\mathrm{F}(000)$

Crystal size

Theta range for data collection

Index ranges

Reflections collected

Independent reflections

Completeness to theta $=27.88^{\circ}$

Absorption correction

Refinement method

Data / restraints / parameters

Goodness-of-fit on $\mathrm{F}^{2}$

Final $\mathrm{R}$ indices [I $>2 \operatorname{sigma}(\mathrm{I})]$

$\mathrm{R}$ indices (all data)

Largest diff. peak and hole ya59sad

C30 H35 N O2 Si

469.68

293(2) K

$0.71073 \AA$

Orthorhombic

Pbca

$a=9.5087(17) \AA$

$\mathrm{a}=90^{\circ}$.

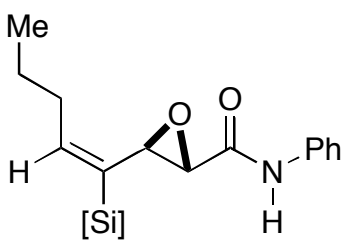

$\mathrm{b}=21.076(4) \AA$

$\mathrm{b}=90^{\circ}$.

$\mathrm{c}=26.118(5) \AA$

$\mathrm{g}=90^{\circ}$.

5234.2(16) $\AA^{3}$

8

$1.192 \mathrm{Mg} / \mathrm{m}^{3}$

$0.116 \mathrm{~mm}^{-1}$

2016

$0.50 \times 0.40 \times 0.30 \mathrm{~mm}^{3}$

1.56 to $27.88^{\circ}$.

$-12<=\mathrm{h}<=12,-27<=\mathrm{k}<=18,-33<=\mathrm{l}<=34$

35328

$6241[\mathrm{R}(\mathrm{int})=0.0970]$

$99.9 \%$

None

Full-matrix least-squares on $\mathrm{F}^{2}$

$6241 / 0 / 387$

1.053

$\mathrm{R} 1=0.0781, \mathrm{wR} 2=0.1760$

$\mathrm{R} 1=0.1235, \mathrm{wR} 2=0.2004$

1.179 and -0.515 e. $\AA^{-3}$ 
Table 2. Atomic coordinates ( $\mathrm{x} 10^{4}$ ) and equivalent isotropic displacement parameters $\left(\AA^{2} \mathrm{x} 10^{3}\right)$ for ya59sad. $U(e q)$ is defined as one third of the trace of the orthogonalized $U^{i j}$ tensor.

\begin{tabular}{|c|c|c|c|c|}
\hline & $\mathrm{x}$ & $\mathrm{y}$ & $\mathrm{z}$ & $\mathrm{U}(\mathrm{eq})$ \\
\hline $\mathrm{C}(1)$ & $8247(3)$ & $10606(1)$ & $2342(1)$ & $26(1)$ \\
\hline$C(2)$ & $6904(3)$ & $11339(1)$ & $1777(1)$ & $26(1)$ \\
\hline$C(3)$ & 7984(4) & $11521(2)$ & $1450(1)$ & $34(1)$ \\
\hline$C(4)$ & $7753(4)$ & 11999(2) & $1096(1)$ & $41(1)$ \\
\hline$C(5)$ & $6469(4)$ & $12299(2)$ & $1063(1)$ & $43(1)$ \\
\hline$C(6)$ & $5399(4)$ & $12119(2)$ & $1383(1)$ & $41(1)$ \\
\hline$C(7)$ & $5610(4)$ & $11645(2)$ & $1743(1)$ & $33(1)$ \\
\hline $\mathrm{C}(8)$ & $8006(3)$ & $10074(2)$ & $2712(1)$ & $29(1)$ \\
\hline $\mathrm{C}(9)$ & $8683(3)$ & $10099(2)$ & $3224(1)$ & $28(1)$ \\
\hline$C(10)$ & $8081(3)$ & $9780(1)$ & $3688(1)$ & $25(1)$ \\
\hline $\mathrm{C}(11)$ & $7194(3)$ & $10108(2)$ & $3982(1)$ & $32(1)$ \\
\hline$C(12)$ & $6684(4)$ & $10775(2)$ & $3873(1)$ & $47(1)$ \\
\hline$C(13)$ & $6144(6)$ & $11126(3)$ & $4331(2)$ & $86(2)$ \\
\hline$C(14)$ & $7122(6)$ & $11241(2)$ & $4746(2)$ & $89(2)$ \\
\hline$C(15)$ & $8177(3)$ & $8383(1)$ & $3374(1)$ & $26(1)$ \\
\hline$C(16)$ & $8799(4)$ & $7786(2)$ & $3286(1)$ & $32(1)$ \\
\hline$C(17)$ & $8250(4)$ & $7365(2)$ & $2928(1)$ & $39(1)$ \\
\hline$C(18)$ & $7062(4)$ & $7525(2)$ & $2653(1)$ & $44(1)$ \\
\hline$C(19)$ & $6412(4)$ & $8101(2)$ & $2742(1)$ & $41(1)$ \\
\hline$C(20)$ & $6966(3)$ & $8524(2)$ & $3094(1)$ & $32(1)$ \\
\hline $\mathrm{C}(21)$ & 10804(3) & $9132(1)$ & $3789(1)$ & $25(1)$ \\
\hline $\mathrm{C}(22)$ & $11722(4)$ & $8747(2)$ & $3518(2)$ & $42(1)$ \\
\hline$C(23)$ & $13135(4)$ & $8909(2)$ & $3462(2)$ & $56(1)$ \\
\hline$C(24)$ & $13657(4)$ & $9454(2)$ & $3678(2)$ & $46(1)$ \\
\hline$C(25)$ & $12771(4)$ & $9839(2)$ & $3953(2)$ & $44(1)$ \\
\hline$C(26)$ & $11356(3)$ & $9684(2)$ & $4005(1)$ & $35(1)$ \\
\hline$C(27)$ & $8407(3)$ & $8682(2)$ & $4518(1)$ & $30(1)$ \\
\hline$C(28)$ & $9264(5)$ & $8070(2)$ & $4606(1)$ & $46(1)$ \\
\hline$C(29)$ & $6830(4)$ & $8519(2)$ & $4560(1)$ & $47(1)$ \\
\hline$C(30)$ & $8803(4)$ & $9152(2)$ & $4942(1)$ & $39(1)$ \\
\hline $\mathrm{N}(1)$ & $7046(3)$ & 10851(1) & $2146(1)$ & $27(1)$ \\
\hline
\end{tabular}




$\begin{array}{lrrrr}\mathrm{O}(1) & 9435(2) & 10794(1) & 2240(1) & 34(1) \\ \mathrm{O}(2) & 9230(2) & 9706(1) & 2824(1) & 39(1) \\ \mathrm{Si}(01) & 8854(1) & 8981(1) & 3848(1) & 23(1)\end{array}$


Table 3. Bond lengths $[\AA ̊]$ and angles $\left[{ }^{\circ}\right]$ for ya59sad.

\begin{tabular}{|c|c|}
\hline $\mathrm{C}(1)-\mathrm{O}(1)$ & $1.228(3)$ \\
\hline $\mathrm{C}(1)-\mathrm{N}(1)$ & $1.355(4)$ \\
\hline $\mathrm{C}(1)-\mathrm{C}(8)$ & $1.497(4)$ \\
\hline$C(2)-C(3)$ & $1.390(4)$ \\
\hline$C(2)-C(7)$ & $1.392(4)$ \\
\hline $\mathrm{C}(2)-\mathrm{N}(1)$ & $1.415(4)$ \\
\hline$C(3)-C(4)$ & $1.384(5)$ \\
\hline$C(4)-C(5)$ & $1.377(5)$ \\
\hline$C(5)-C(6)$ & $1.372(5)$ \\
\hline$C(6)-C(7)$ & $1.386(5)$ \\
\hline $\mathrm{C}(8)-\mathrm{O}(2)$ & $1.428(4)$ \\
\hline $\mathrm{C}(8)-\mathrm{C}(9)$ & $1.485(4)$ \\
\hline $\mathrm{C}(9)-\mathrm{O}(2)$ & $1.430(4)$ \\
\hline $\mathrm{C}(9)-\mathrm{C}(10)$ & $1.500(4)$ \\
\hline$C(10)-C(11)$ & $1.332(4)$ \\
\hline $\mathrm{C}(10)-\mathrm{Si}(01)$ & $1.884(3)$ \\
\hline $\mathrm{C}(11)-\mathrm{C}(12)$ & $1.515(5)$ \\
\hline $\mathrm{C}(12)-\mathrm{C}(13)$ & $1.495(6)$ \\
\hline $\mathrm{C}(13)-\mathrm{C}(14)$ & $1.448(7)$ \\
\hline$C(15)-C(20)$ & $1.396(4)$ \\
\hline$C(15)-C(16)$ & $1.409(4)$ \\
\hline $\mathrm{C}(15)-\mathrm{Si}(01)$ & $1.880(3)$ \\
\hline$C(16)-C(17)$ & $1.392(5)$ \\
\hline $\mathrm{C}(17)-\mathrm{C}(18)$ & $1.382(5)$ \\
\hline $\mathrm{C}(18)-\mathrm{C}(19)$ & $1.382(5)$ \\
\hline$C(19)-C(20)$ & $1.384(5)$ \\
\hline$C(21)-C(22)$ & $1.386(5)$ \\
\hline$C(21)-C(26)$ & $1.396(4)$ \\
\hline $\mathrm{C}(21)-\mathrm{Si}(01)$ & $1.887(3)$ \\
\hline $\mathrm{C}(22)-\mathrm{C}(23)$ & $1.393(5)$ \\
\hline$C(23)-C(24)$ & $1.373(6)$ \\
\hline$C(24)-C(25)$ & $1.373(5)$ \\
\hline$C(25)-C(26)$ & $1.391(5)$ \\
\hline$C(27)-C(30)$ & $1.534(4)$ \\
\hline
\end{tabular}




\begin{tabular}{|c|c|}
\hline$C(27)-C(29)$ & $1.542(5)$ \\
\hline $\mathrm{C}(27)-\mathrm{C}(28)$ & $1.543(5)$ \\
\hline $\mathrm{C}(27)-\mathrm{Si}(01)$ & $1.909(3)$ \\
\hline $\mathrm{O}(1)-\mathrm{C}(1)-\mathrm{N}(1)$ & $124.7(3)$ \\
\hline $\mathrm{O}(1)-\mathrm{C}(1)-\mathrm{C}(8)$ & $121.6(3)$ \\
\hline $\mathrm{N}(1)-\mathrm{C}(1)-\mathrm{C}(8)$ & $113.7(3)$ \\
\hline$C(3)-C(2)-C(7)$ & 119.1(3) \\
\hline $\mathrm{C}(3)-\mathrm{C}(2)-\mathrm{N}(1)$ & $123.3(3)$ \\
\hline$C(7)-C(2)-N(1)$ & $117.7(3)$ \\
\hline$C(4)-C(3)-C(2)$ & 119.6(3) \\
\hline$C(5)-C(4)-C(3)$ & 121.1(4) \\
\hline$C(6)-C(5)-C(4)$ & $119.5(3)$ \\
\hline$C(5)-C(6)-C(7)$ & $120.3(4)$ \\
\hline$C(6)-C(7)-C(2)$ & $120.4(3)$ \\
\hline $\mathrm{O}(2)-\mathrm{C}(8)-\mathrm{C}(9)$ & $58.77(19)$ \\
\hline $\mathrm{O}(2)-\mathrm{C}(8)-\mathrm{C}(1)$ & $114.4(3)$ \\
\hline $\mathrm{C}(9)-\mathrm{C}(8)-\mathrm{C}(1)$ & 119.1(3) \\
\hline $\mathrm{O}(2)-\mathrm{C}(9)-\mathrm{C}(8)$ & $58.63(19)$ \\
\hline $\mathrm{O}(2)-\mathrm{C}(9)-\mathrm{C}(10)$ & 118.1(3) \\
\hline $\mathrm{C}(8)-\mathrm{C}(9)-\mathrm{C}(10)$ & 123.1(3) \\
\hline $\mathrm{C}(11)-\mathrm{C}(10)-\mathrm{C}(9)$ & 118.3(3) \\
\hline$C(11)-C(10)-\operatorname{Si}(01)$ & $125.7(2)$ \\
\hline $\mathrm{C}(9)-\mathrm{C}(10)-\mathrm{Si}(01)$ & $115.4(2)$ \\
\hline $\mathrm{C}(10)-\mathrm{C}(11)-\mathrm{C}(12)$ & $125.2(3)$ \\
\hline $\mathrm{C}(13)-\mathrm{C}(12)-\mathrm{C}(11)$ & $114.8(4)$ \\
\hline$C(14)-C(13)-C(12)$ & $117.4(5)$ \\
\hline$C(20)-C(15)-C(16)$ & $116.8(3)$ \\
\hline$C(20)-C(15)-\operatorname{Si}(01)$ & $119.0(2)$ \\
\hline$C(16)-C(15)-S i(01)$ & $124.2(2)$ \\
\hline$C(17)-C(16)-C(15)$ & $121.5(3)$ \\
\hline $\mathrm{C}(18)-\mathrm{C}(17)-\mathrm{C}(16)$ & $120.1(3)$ \\
\hline $\mathrm{C}(17)-\mathrm{C}(18)-\mathrm{C}(19)$ & $119.5(3)$ \\
\hline $\mathrm{C}(18)-\mathrm{C}(19)-\mathrm{C}(20)$ & $120.5(4)$ \\
\hline$C(19)-C(20)-C(15)$ & $121.7(3)$ \\
\hline $\mathrm{C}(22)-\mathrm{C}(21)-\mathrm{C}(26)$ & $117.2(3)$ \\
\hline
\end{tabular}




$\begin{array}{lc}\mathrm{C}(22)-\mathrm{C}(21)-\mathrm{Si}(01) & 124.2(2) \\ \mathrm{C}(26)-\mathrm{C}(21)-\mathrm{Si}(01) & 118.5(2) \\ \mathrm{C}(21)-\mathrm{C}(22)-\mathrm{C}(23) & 121.2(3) \\ \mathrm{C}(24)-\mathrm{C}(23)-\mathrm{C}(22) & 120.6(4) \\ \mathrm{C}(23)-\mathrm{C}(24)-\mathrm{C}(25) & 119.2(3) \\ \mathrm{C}(24)-\mathrm{C}(25)-\mathrm{C}(26) & 120.4(3) \\ \mathrm{C}(25)-\mathrm{C}(26)-\mathrm{C}(21) & 121.3(3) \\ \mathrm{C}(30)-\mathrm{C}(27)-\mathrm{C}(29) & 109.4(3) \\ \mathrm{C}(30)-\mathrm{C}(27)-\mathrm{C}(28) & 107.7(3) \\ \mathrm{C}(29)-\mathrm{C}(27)-\mathrm{C}(28) & 108.5(3) \\ \mathrm{C}(30)-\mathrm{C}(27)-\mathrm{Si}(01) & 113.2(2) \\ \mathrm{C}(29)-\mathrm{C}(27)-\mathrm{Si}(01) & 110.8(2) \\ \mathrm{C}(28)-\mathrm{C}(27)-\mathrm{Si}(01) & 107.1(2) \\ \mathrm{C}(1)-\mathrm{N}(1)-\mathrm{C}(2) & 128.1(3) \\ \mathrm{C}(8)-\mathrm{O}(2)-\mathrm{C}(9) & 62.6(2) \\ \mathrm{C}(15)-\mathrm{Si}(01)-\mathrm{C}(10) & 108.65(13) \\ \mathrm{C}(15)-\mathrm{Si}(01)-\mathrm{C}(21) & 113.32(13) \\ \mathrm{C}(10)-\mathrm{Si}(01)-\mathrm{C}(21) & 102.46(13) \\ \mathrm{C}(15)-\mathrm{Si}(01)-\mathrm{C}(27) & 107.79(14) \\ \mathrm{C}(10)-\mathrm{Si}(01)-\mathrm{C}(27) & 114.30(14) \\ \mathrm{C}(21)-\mathrm{Si}(01)-\mathrm{C}(27) & 110.40(14)\end{array}$

Symmetry transformations used to generate equivalent atoms: 
Table 4. Anisotropic displacement parameters $\left(\AA^{2} \times 10^{3}\right)$ for ya59sad. The anisotropic displacement factor exponent takes the form: $-2 \mathrm{p}^{2}\left[\mathrm{~h}^{2} \mathrm{a}^{* 2} \mathrm{U}^{11}+\ldots+2 \mathrm{hk} \mathrm{a}^{*} \mathrm{~b}^{*} \mathrm{U}^{12}\right]$

\begin{tabular}{|c|c|c|c|c|c|c|}
\hline & $\mathrm{U}^{11}$ & $\mathrm{U}^{22}$ & $\mathrm{U}^{33}$ & $\mathrm{U}^{23}$ & $\mathrm{U}^{13}$ & $\mathrm{U}^{12}$ \\
\hline $\mathrm{C}(1)$ & $23(2)$ & $29(2)$ & $25(2)$ & $-2(1)$ & $0(1)$ & $-2(1)$ \\
\hline$C(2)$ & $29(2)$ & $24(2)$ & $24(2)$ & $-1(1)$ & $-1(1)$ & $-5(1)$ \\
\hline$C(3)$ & $33(2)$ & $38(2)$ & $32(2)$ & $-1(1)$ & $-2(1)$ & $-2(2)$ \\
\hline$C(4)$ & $50(2)$ & 43(2) & $31(2)$ & $4(2)$ & $5(2)$ & $-10(2)$ \\
\hline$C(5)$ & $59(3)$ & $35(2)$ & $34(2)$ & $5(2)$ & $-8(2)$ & $1(2)$ \\
\hline$C(6)$ & $46(2)$ & $35(2)$ & $43(2)$ & $-2(2)$ & $-7(2)$ & $8(2)$ \\
\hline$C(7)$ & $34(2)$ & $29(2)$ & $35(2)$ & $1(1)$ & $-3(1)$ & $2(1)$ \\
\hline$C(8)$ & $22(2)$ & $31(2)$ & $33(2)$ & $1(1)$ & $-3(1)$ & $0(1)$ \\
\hline $\mathrm{C}(9)$ & $25(2)$ & $28(2)$ & $32(2)$ & $-1(1)$ & $-3(1)$ & $-1(1)$ \\
\hline$C(10)$ & $22(2)$ & $25(2)$ & $27(2)$ & $-1(1)$ & $-4(1)$ & $-1(1)$ \\
\hline$C(11)$ & $32(2)$ & $36(2)$ & $30(2)$ & $0(1)$ & $0(1)$ & $4(1)$ \\
\hline$C(12)$ & $52(2)$ & $40(2)$ & $47(2)$ & $-1(2)$ & $-4(2)$ & $22(2)$ \\
\hline$C(13)$ & $95(4)$ & $68(3)$ & $94(4)$ & $-23(3)$ & $-31(3)$ & $33(3)$ \\
\hline$C(14)$ & $97(4)$ & $70(3)$ & $102(4)$ & $-34(3)$ & $-47(4)$ & $27(3)$ \\
\hline$C(15)$ & $24(2)$ & $26(2)$ & $26(2)$ & $-1(1)$ & $3(1)$ & $-6(1)$ \\
\hline$C(16)$ & $32(2)$ & $29(2)$ & $35(2)$ & $-4(1)$ & $8(1)$ & $-6(1)$ \\
\hline $\mathrm{C}(17)$ & $41(2)$ & $34(2)$ & $41(2)$ & $-14(2)$ & $14(2)$ & $-5(2)$ \\
\hline $\mathrm{C}(18)$ & $43(2)$ & 48(2) & $40(2)$ & $-20(2)$ & $8(2)$ & $-16(2)$ \\
\hline $\mathrm{C}(19)$ & $29(2)$ & $56(2)$ & $39(2)$ & $-7(2)$ & $-5(2)$ & $-11(2)$ \\
\hline $\mathrm{C}(20)$ & $29(2)$ & $35(2)$ & $33(2)$ & $-3(1)$ & $0(1)$ & $-4(1)$ \\
\hline $\mathrm{C}(21)$ & $23(2)$ & $25(2)$ & $26(2)$ & $1(1)$ & $-3(1)$ & $0(1)$ \\
\hline $\mathrm{C}(22)$ & $29(2)$ & $36(2)$ & $60(2)$ & $-13(2)$ & $6(2)$ & $-5(2)$ \\
\hline $\mathrm{C}(23)$ & $31(2)$ & $52(3)$ & $85(3)$ & $-13(2)$ & $17(2)$ & $1(2)$ \\
\hline$C(24)$ & $21(2)$ & 49(2) & $68(3)$ & $4(2)$ & $2(2)$ & $-6(2)$ \\
\hline$C(25)$ & $33(2)$ & 41(2) & $58(2)$ & $-6(2)$ & $-8(2)$ & $-9(2)$ \\
\hline$C(26)$ & $27(2)$ & $34(2)$ & $42(2)$ & $-6(2)$ & $1(1)$ & $-2(1)$ \\
\hline$C(27)$ & $37(2)$ & $28(2)$ & $25(2)$ & $-1(1)$ & $2(1)$ & $-3(1)$ \\
\hline$C(28)$ & $73(3)$ & $31(2)$ & $34(2)$ & $7(2)$ & $2(2)$ & $7(2)$ \\
\hline$C(29)$ & $46(2)$ & $55(2)$ & $39(2)$ & $1(2)$ & $11(2)$ & $-17(2)$ \\
\hline $\mathrm{C}(30)$ & $50(2)$ & $37(2)$ & $31(2)$ & $-2(1)$ & $-1(2)$ & $-2(2)$ \\
\hline $\mathrm{N}(1)$ & $20(1)$ & $32(2)$ & $30(1)$ & $5(1)$ & $-2(1)$ & $-2(1)$ \\
\hline
\end{tabular}




$\begin{array}{lllllll}\mathrm{O}(1) & 18(1) & 46(1) & 39(1) & 8(1) & -1(1) & -6(1) \\ \mathrm{O}(2) & 37(1) & 44(1) & 35(1) & 5(1) & 4(1) & 17(1) \\ \mathrm{Si}(01) & 22(1) & 23(1) & 24(1) & -2(1) & -1(1) & -1(1)\end{array}$


Table 5. Hydrogen coordinates ( $\left.\mathrm{x} 10^{4}\right)$ and isotropic displacement parameters $\left(\AA^{2} \times 10^{3}\right)$ for ya59sad.

\begin{tabular}{|c|c|c|c|c|}
\hline & $\mathrm{x}$ & $\mathrm{y}$ & $\mathrm{z}$ & $\mathrm{U}(\mathrm{eq})$ \\
\hline $\mathrm{H}(12 \mathrm{~A})$ & 7453 & 11016 & 3725 & 56 \\
\hline $\mathrm{H}(12 \mathrm{~B})$ & 5940 & 10754 & 3620 & 56 \\
\hline $\mathrm{H}(13 \mathrm{~A})$ & 5789 & 11533 & 4215 & 103 \\
\hline $\mathrm{H}(13 \mathrm{~B})$ & 5352 & 10891 & 4467 & 103 \\
\hline $\mathrm{H}(14 \mathrm{~A})$ & 7359 & 10846 & 4906 & 134 \\
\hline $\mathrm{H}(14 \mathrm{~B})$ & 6695 & 11518 & 4992 & 134 \\
\hline $\mathrm{H}(14 \mathrm{C})$ & 7959 & 11435 & 4613 & 134 \\
\hline $\mathrm{H}(28 \mathrm{~A})$ & 10246 & 8158 & 4559 & 69 \\
\hline $\mathrm{H}(28 \mathrm{~B})$ & 8972 & 7752 & 4365 & 69 \\
\hline $\mathrm{H}(28 \mathrm{C})$ & 9107 & 7919 & 4947 & 69 \\
\hline $\mathrm{H}(29 \mathrm{~A})$ & 6641 & 8339 & 4890 & 70 \\
\hline $\mathrm{H}(29 \mathrm{~B})$ & 6585 & 8218 & 4298 & 70 \\
\hline $\mathrm{H}(29 \mathrm{C})$ & 6283 & 8898 & 4516 & 70 \\
\hline $\mathrm{H}(30 \mathrm{~A})$ & 8614 & 8965 & 5270 & 59 \\
\hline $\mathrm{H}(30 \mathrm{~B})$ & 8258 & 9532 & 4905 & 59 \\
\hline $\mathrm{H}(30 \mathrm{C})$ & 9785 & 9253 & 4918 & 59 \\
\hline $\mathrm{H}(3)$ & $8820(40)$ & $11339(16)$ & $1507(12)$ & $29(9)$ \\
\hline $\mathrm{H}(4)$ & $8500(40)$ & $12095(19)$ & $880(16)$ & $55(12)$ \\
\hline $\mathrm{H}(5)$ & $6330(40)$ & $12635(17)$ & $809(15)$ & $46(10)$ \\
\hline $\mathrm{H}(6)$ & $4510(50)$ & $12346(19)$ & $1390(16)$ & $60(12)$ \\
\hline $\mathrm{H}(7)$ & $4920(40)$ & $11537(16)$ & 1985(14) & $41(10)$ \\
\hline $\mathrm{H}(8)$ & $7190(30)$ & $9837(14)$ & $2672(11)$ & $22(8)$ \\
\hline $\mathrm{H}(9)$ & $9220(30)$ & $10472(15)$ & $3293(12)$ & $28(8)$ \\
\hline $\mathrm{H}(11)$ & $6930(30)$ & $9937(13)$ & $4296(12)$ & $17(7)$ \\
\hline $\mathrm{H}(16)$ & $9660(40)$ & $7648(17)$ & $3484(14)$ & $49(11)$ \\
\hline $\mathrm{H}(17)$ & $8670(40)$ & $6946(18)$ & 2883(13) & $42(10)$ \\
\hline $\mathrm{H}(18)$ & $6670(40)$ & $7233(18)$ & $2386(15)$ & $50(11)$ \\
\hline $\mathrm{H}(19)$ & $5600(40)$ & $8205(17)$ & $2590(15)$ & $49(11)$ \\
\hline $\mathrm{H}(20)$ & $6520(40)$ & 8931(19) & $3127(15)$ & $53(12)$ \\
\hline $\mathrm{H}(22)$ & $11390(40)$ & $8350(18)$ & $3373(14)$ & $47(11)$ \\
\hline
\end{tabular}




\begin{tabular}{lrrrr}
$\mathrm{H}(23)$ & $13700(50)$ & $8660(20)$ & $3268(18)$ & $79(16)$ \\
$\mathrm{H}(24)$ & $14630(40)$ & $9592(19)$ & $3628(15)$ & $53(11)$ \\
$\mathrm{H}(25)$ & $13180(40)$ & $10260(20)$ & $4120(16)$ & $64(13)$ \\
$\mathrm{H}(26)$ & $10730(40)$ & $9962(18)$ & $4207(15)$ & $52(11)$ \\
$\mathrm{H}(1 \mathrm{~N})$ & $6300(50)$ & $10720(20)$ & $2268(16)$ & $59(13)$ \\
\hline
\end{tabular}


Table 6. Torsion angles $\left[{ }^{\circ}\right]$ for ya59sad.

\begin{tabular}{|c|c|}
\hline $\mathrm{C}(7)-\mathrm{C}(2)-\mathrm{C}(3)-\mathrm{C}(4)$ & $-0.2(5)$ \\
\hline $\mathrm{N}(1)-\mathrm{C}(2)-\mathrm{C}(3)-\mathrm{C}(4)$ & $179.3(3)$ \\
\hline$C(2)-C(3)-C(4)-C(5)$ & $0.0(5)$ \\
\hline$C(3)-C(4)-C(5)-C(6)$ & $-0.3(6)$ \\
\hline$C(4)-C(5)-C(6)-C(7)$ & $0.8(5)$ \\
\hline$C(5)-C(6)-C(7)-C(2)$ & $-1.0(5)$ \\
\hline $\mathrm{C}(3)-\mathrm{C}(2)-\mathrm{C}(7)-\mathrm{C}(6)$ & $0.7(5)$ \\
\hline $\mathrm{N}(1)-\mathrm{C}(2)-\mathrm{C}(7)-\mathrm{C}(6)$ & $-178.9(3)$ \\
\hline $\mathrm{O}(1)-\mathrm{C}(1)-\mathrm{C}(8)-\mathrm{O}(2)$ & $-15.6(4)$ \\
\hline $\mathrm{N}(1)-\mathrm{C}(1)-\mathrm{C}(8)-\mathrm{O}(2)$ & $165.0(3)$ \\
\hline $\mathrm{O}(1)-\mathrm{C}(1)-\mathrm{C}(8)-\mathrm{C}(9)$ & $50.9(4)$ \\
\hline $\mathrm{N}(1)-\mathrm{C}(1)-\mathrm{C}(8)-\mathrm{C}(9)$ & $-128.5(3)$ \\
\hline $\mathrm{C}(1)-\mathrm{C}(8)-\mathrm{C}(9)-\mathrm{O}(2)$ & $-102.4(3)$ \\
\hline $\mathrm{O}(2)-\mathrm{C}(8)-\mathrm{C}(9)-\mathrm{C}(10)$ & $-105.1(3)$ \\
\hline $\mathrm{C}(1)-\mathrm{C}(8)-\mathrm{C}(9)-\mathrm{C}(10)$ & $152.5(3)$ \\
\hline $\mathrm{O}(2)-\mathrm{C}(9)-\mathrm{C}(10)-\mathrm{C}(11)$ & $-159.3(3)$ \\
\hline $\mathrm{C}(8)-\mathrm{C}(9)-\mathrm{C}(10)-\mathrm{C}(11)$ & $-90.2(4)$ \\
\hline $\mathrm{O}(2)-\mathrm{C}(9)-\mathrm{C}(10)-\mathrm{Si}(01)$ & $28.6(3)$ \\
\hline $\mathrm{C}(8)-\mathrm{C}(9)-\mathrm{C}(10)-\mathrm{Si}(01)$ & $97.8(3)$ \\
\hline $\mathrm{C}(9)-\mathrm{C}(10)-\mathrm{C}(11)-\mathrm{C}(12)$ & $2.9(5)$ \\
\hline $\mathrm{Si}(01)-\mathrm{C}(10)-\mathrm{C}(11)-\mathrm{C}(12)$ & $174.0(3)$ \\
\hline $\mathrm{C}(10)-\mathrm{C}(11)-\mathrm{C}(12)-\mathrm{C}(13)$ & $-158.7(4)$ \\
\hline $\mathrm{C}(11)-\mathrm{C}(12)-\mathrm{C}(13)-\mathrm{C}(14)$ & $61.6(7)$ \\
\hline$C(20)-C(15)-C(16)-C(17)$ & $1.5(5)$ \\
\hline $\mathrm{Si}(01)-\mathrm{C}(15)-\mathrm{C}(16)-\mathrm{C}(17)$ & $-179.7(2)$ \\
\hline$C(15)-C(16)-C(17)-C(18)$ & $-0.6(5)$ \\
\hline $\mathrm{C}(16)-\mathrm{C}(17)-\mathrm{C}(18)-\mathrm{C}(19)$ & $-1.2(5)$ \\
\hline $\mathrm{C}(17)-\mathrm{C}(18)-\mathrm{C}(19)-\mathrm{C}(20)$ & $1.9(6)$ \\
\hline$C(18)-C(19)-C(20)-C(15)$ & $-0.9(5)$ \\
\hline $\mathrm{C}(16)-\mathrm{C}(15)-\mathrm{C}(20)-\mathrm{C}(19)$ & $-0.8(5)$ \\
\hline $\mathrm{Si}(01)-\mathrm{C}(15)-\mathrm{C}(20)-\mathrm{C}(19)$ & $-179.6(3)$ \\
\hline$C(26)-C(21)-C(22)-C(23)$ & $-0.2(6)$ \\
\hline $\mathrm{Si}(01)-\mathrm{C}(21)-\mathrm{C}(22)-\mathrm{C}(23)$ & $175.7(3)$ \\
\hline $\mathrm{C}(21)-\mathrm{C}(22)-\mathrm{C}(23)-\mathrm{C}(24)$ & $0.4(7)$ \\
\hline
\end{tabular}




\begin{tabular}{|c|c|}
\hline$C(22)-C(23)-C(24)-C(25)$ & $0.2(7)$ \\
\hline$C(23)-C(24)-C(25)-C(26)$ & $-1.0(6)$ \\
\hline$C(24)-C(25)-C(26)-C(21)$ & $1.3(6)$ \\
\hline $\mathrm{C}(22)-\mathrm{C}(21)-\mathrm{C}(26)-\mathrm{C}(25)$ & $-0.6(5)$ \\
\hline $\mathrm{Si}(01)-\mathrm{C}(21)-\mathrm{C}(26)-\mathrm{C}(25)$ & $-176.7(3)$ \\
\hline $\mathrm{O}(1)-\mathrm{C}(1)-\mathrm{N}(1)-\mathrm{C}(2)$ & $3.0(5)$ \\
\hline $\mathrm{C}(8)-\mathrm{C}(1)-\mathrm{N}(1)-\mathrm{C}(2)$ & $-177.7(3)$ \\
\hline $\mathrm{C}(3)-\mathrm{C}(2)-\mathrm{N}(1)-\mathrm{C}(1)$ & $19.3(5)$ \\
\hline $\mathrm{C}(7)-\mathrm{C}(2)-\mathrm{N}(1)-\mathrm{C}(1)$ & $-161.2(3)$ \\
\hline $\mathrm{C}(1)-\mathrm{C}(8)-\mathrm{O}(2)-\mathrm{C}(9)$ & $110.5(3)$ \\
\hline $\mathrm{C}(10)-\mathrm{C}(9)-\mathrm{O}(2)-\mathrm{C}(8)$ & $113.6(3)$ \\
\hline$C(20)-C(15)-S i(01)-C(10)$ & $-19.1(3)$ \\
\hline$C(16)-C(15)-S i(01)-C(10)$ & $162.2(3)$ \\
\hline$C(20)-C(15)-S i(01)-C(21)$ & $-132.3(2)$ \\
\hline$C(16)-C(15)-\operatorname{Si}(01)-C(21)$ & $49.0(3)$ \\
\hline$C(20)-C(15)-S i(01)-C(27)$ & $105.2(3)$ \\
\hline$C(16)-C(15)-S i(01)-C(27)$ & $-73.5(3)$ \\
\hline $\mathrm{C}(11)-\mathrm{C}(10)-\mathrm{Si}(01)-\mathrm{C}(15)$ & $114.8(3)$ \\
\hline $\mathrm{C}(9)-\mathrm{C}(10)-\mathrm{Si}(01)-\mathrm{C}(15)$ & $-73.8(2)$ \\
\hline $\mathrm{C}(11)-\mathrm{C}(10)-\mathrm{Si}(01)-\mathrm{C}(21)$ & $-125.0(3)$ \\
\hline $\mathrm{C}(9)-\mathrm{C}(10)-\mathrm{Si}(01)-\mathrm{C}(21)$ & $46.3(2)$ \\
\hline $\mathrm{C}(11)-\mathrm{C}(10)-\mathrm{Si}(01)-\mathrm{C}(27)$ & $-5.6(3)$ \\
\hline $\mathrm{C}(9)-\mathrm{C}(10)-\mathrm{Si}(01)-\mathrm{C}(27)$ & $165.8(2)$ \\
\hline $\mathrm{C}(22)-\mathrm{C}(21)-\mathrm{Si}(01)-\mathrm{C}(15)$ & $-14.5(3)$ \\
\hline$C(26)-C(21)-S i(01)-C(15)$ & $161.3(2)$ \\
\hline $\mathrm{C}(22)-\mathrm{C}(21)-\mathrm{Si}(01)-\mathrm{C}(10)$ & $-131.4(3)$ \\
\hline$C(26)-C(21)-S i(01)-C(10)$ & $44.5(3)$ \\
\hline $\mathrm{C}(22)-\mathrm{C}(21)-\mathrm{Si}(01)-\mathrm{C}(27)$ & $106.5(3)$ \\
\hline$C(26)-C(21)-S i(01)-C(27)$ & $-77.6(3)$ \\
\hline $\mathrm{C}(30)-\mathrm{C}(27)-\mathrm{Si}(01)-\mathrm{C}(15)$ & $-175.6(2)$ \\
\hline$C(29)-C(27)-S i(01)-C(15)$ & $-52.3(3)$ \\
\hline $\mathrm{C}(28)-\mathrm{C}(27)-\mathrm{Si}(01)-\mathrm{C}(15)$ & $65.8(3)$ \\
\hline$C(30)-C(27)-S i(01)-C(10)$ & $-54.7(3)$ \\
\hline $\mathrm{C}(29)-\mathrm{C}(27)-\mathrm{Si}(01)-\mathrm{C}(10)$ & $68.6(3)$ \\
\hline $\mathrm{C}(28)-\mathrm{C}(27)-\mathrm{Si}(01)-\mathrm{C}(10)$ & $-173.3(2)$ \\
\hline $\mathrm{C}(30)-\mathrm{C}(27)-\mathrm{Si}(01)-\mathrm{C}(21)$ & $60.2(3)$ \\
\hline
\end{tabular}


$\mathrm{C}(29)-\mathrm{C}(27)-\mathrm{Si}(01)-\mathrm{C}(21)$

$-176.6(2)$

$\mathrm{C}(28)-\mathrm{C}(27)-\mathrm{Si}(01)-\mathrm{C}(21)$

$-58.4(3)$ 
Table 1. Crystal data and structure refinement for ya58sad.

Identification code

Empirical formula

Formula weight

Temperature

Wavelength

Crystal system

Space group

Unit cell dimensions

Volume

Z

Density (calculated)

Absorption coefficient

$\mathrm{F}(000)$

Crystal size

Theta range for data collection

Index ranges

Reflections collected

Independent reflections

Completeness to theta $=27.95^{\circ}$

Absorption correction

Max. and min. transmission

Refinement method

Data / restraints / parameters

Goodness-of-fit on $\mathrm{F}^{2}$

Final $\mathrm{R}$ indices [I $>2 \operatorname{sigma}(\mathrm{I})]$

$\mathrm{R}$ indices (all data)

Largest diff. peak and hole ya58sad

C31 H37 N O2 Si

483.71

193(2) K

$0.71073 \AA$

Orthorhombic

Pbca

$a=9.6596(16) \AA$

$\mathrm{b}=21.302(4) \AA$

$c=26.248(4) \AA$

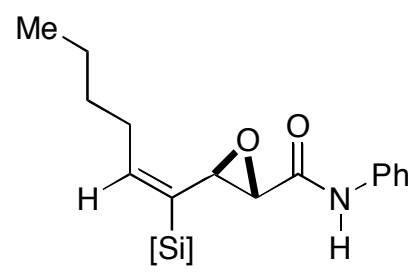

(土)

(Table 2, entry 4)

5401.1(16) $\AA^{3}$

8

$1.190 \mathrm{Mg} / \mathrm{m}^{3}$

$0.115 \mathrm{~mm}^{-1}$

2080

$0.32 \times 0.10 \times 0.02 \mathrm{~mm}^{3}$

1.55 to $27.95^{\circ}$.

$-5<=\mathrm{h}<=12,-27<=\mathrm{k}<=28,-34<=\mathrm{l}<=34$

36172

$6460[\mathrm{R}($ int $)=0.0699]$

$99.6 \%$

None

0.9977 and 0.9642

Full-matrix least-squares on $\mathrm{F}^{2}$

$6460 / 0$ / 388

1.074

$\mathrm{R} 1=0.0699, \mathrm{wR} 2=0.1525$

$\mathrm{R} 1=0.1010, \mathrm{wR} 2=0.1689$

0.840 and -0.596 e. $\AA^{-3}$ 
Table 2. Atomic coordinates ( $\mathrm{x} 10^{4}$ ) and equivalent isotropic displacement parameters $\left(\AA^{2} \mathrm{x} 10^{3}\right)$ for ya58sad. $U(e q)$ is defined as one third of the trace of the orthogonalized $U^{i j}$ tensor.

\begin{tabular}{|c|c|c|c|c|}
\hline & $\mathrm{x}$ & $\mathrm{y}$ & $\mathrm{z}$ & $\mathrm{U}(\mathrm{eq})$ \\
\hline$C(1)$ & $1667(2)$ & $672(1)$ & $2341(1)$ & $26(1)$ \\
\hline $\mathrm{C}(2)$ & $1888(3)$ & $1213(1)$ & $2699(1)$ & $28(1)$ \\
\hline$C(3)$ & $1242(3)$ & $1195(1)$ & $3209(1)$ & $30(1)$ \\
\hline$C(4)$ & $1838(2)$ & $1535(1)$ & $3658(1)$ & $25(1)$ \\
\hline$C(5)$ & $2812(3)$ & $1251(1)$ & $3935(1)$ & $31(1)$ \\
\hline$C(6)$ & $3407(3)$ & $608(1)$ & $3847(1)$ & $44(1)$ \\
\hline$C(7)$ & $3327(6)$ & 192(2) & $4291(2)$ & $105(2)$ \\
\hline $\mathrm{C}(8)$ & $2162(7)$ & $95(2)$ & $4571(2)$ & $138(3)$ \\
\hline$C(10)$ & $-898(2)$ & $2157(1)$ & $3744(1)$ & $26(1)$ \\
\hline$C(11)$ & $-1796(3)$ & $2541(1)$ & $3474(1)$ & $44(1)$ \\
\hline$C(12)$ & $-3185(3)$ & $2394(2)$ & $3419(2)$ & $56(1)$ \\
\hline$C(13)$ & $-3713(3)$ & $1864(2)$ & $3635(1)$ & $45(1)$ \\
\hline$C(14)$ & $-2855(3)$ & $1477(1)$ & $3912(1)$ & $42(1)$ \\
\hline$C(15)$ & $-1468(3)$ & $1622(1)$ & $3964(1)$ & $34(1)$ \\
\hline$C(16)$ & $1417(3)$ & $2600(1)$ & $4483(1)$ & $27(1)$ \\
\hline$C(17)$ & 2893(3) & $2859(1)$ & $4511(1)$ & $38(1)$ \\
\hline$C(18)$ & $1241(3)$ & $2095(1)$ & $4895(1)$ & $33(1)$ \\
\hline$C(19)$ & $405(3)$ & $3141(1)$ & $4606(1)$ & $38(1)$ \\
\hline$C(20)$ & $1682(3)$ & 2919(1) & $3354(1)$ & $26(1)$ \\
\hline $\mathrm{C}(21)$ & $1083(3)$ & $3512(1)$ & $3287(1)$ & $33(1)$ \\
\hline$C(22)$ & $1660(3)$ & $3950(1)$ & $2956(1)$ & $43(1)$ \\
\hline$C(23)$ & $2855(3)$ & $3813(2)$ & $2696(1)$ & $46(1)$ \\
\hline$C(24)$ & $3490(3)$ & $3239(2)$ & $2764(1)$ & $43(1)$ \\
\hline$C(25)$ & $2902(3)$ & 2799(1) & $3086(1)$ & $32(1)$ \\
\hline$C(26)$ & 2997(3) & $-50(1)$ & 1781(1) & $27(1)$ \\
\hline$C(27)$ & 1941(3) & $-213(1)$ & $1449(1)$ & $36(1)$ \\
\hline$C(28)$ & $2174(4)$ & $-677(1)$ & 1089(1) & $45(1)$ \\
\hline$C(29)$ & $3436(4)$ & $-976(1)$ & $1056(1)$ & $49(1)$ \\
\hline$C(30)$ & $4477(4)$ & $-816(1)$ & $1385(1)$ & $45(1)$ \\
\hline$C(31)$ & $4264(3)$ & $-351(1)$ & 1751(1) & $34(1)$ \\
\hline $\mathrm{N}(1)$ & $2848(2)$ & $434(1)$ & $2150(1)$ & $27(1)$ \\
\hline
\end{tabular}




\begin{tabular}{lrrrr}
$\mathrm{O}(1)$ & $506(2)$ & $476(1)$ & $2245(1)$ & $35(1)$ \\
$\mathrm{O}(2)$ & $670(2)$ & $1566(1)$ & $2805(1)$ & $43(1)$ \\
$\mathrm{Si}(1)$ & $1012(1)$ & $2311(1)$ & $3812(1)$ & $22(1)$ \\
$\mathrm{C}(9)$ & $1935(6)$ & $-365(2)$ & $4969(2)$ & $105(2)$ \\
\hline
\end{tabular}


Table 3. Bond lengths $[\AA]$ and angles $\left[{ }^{\circ}\right]$ for ya58sad.

\begin{tabular}{|c|c|}
\hline $\mathrm{C}(1)-\mathrm{O}(1)$ & $1.223(3)$ \\
\hline $\mathrm{C}(1)-\mathrm{N}(1)$ & $1.345(3)$ \\
\hline $\mathrm{C}(1)-\mathrm{C}(2)$ & $1.503(3)$ \\
\hline $\mathrm{C}(2)-\mathrm{O}(2)$ & $1.424(3)$ \\
\hline$C(2)-C(3)$ & $1.477(3)$ \\
\hline $\mathrm{C}(3)-\mathrm{O}(2)$ & $1.432(3)$ \\
\hline$C(3)-C(4)$ & $1.498(3)$ \\
\hline$C(4)-C(5)$ & $1.335(3)$ \\
\hline $\mathrm{C}(4)-\mathrm{Si}(1)$ & $1.881(2)$ \\
\hline$C(5)-C(6)$ & $1.502(4)$ \\
\hline$C(6)-C(7)$ & $1.467(5)$ \\
\hline$C(7)-C(8)$ & $1.360(7)$ \\
\hline $\mathrm{C}(8)-\mathrm{C}(9)$ & $1.449(6)$ \\
\hline$C(10)-C(11)$ & $1.387(4)$ \\
\hline $\mathrm{C}(10)-\mathrm{C}(15)$ & $1.392(3)$ \\
\hline $\mathrm{C}(10)-\mathrm{Si}(1)$ & $1.882(2)$ \\
\hline $\mathrm{C}(11)-\mathrm{C}(12)$ & $1.386(4)$ \\
\hline $\mathrm{C}(12)-\mathrm{C}(13)$ & $1.362(4)$ \\
\hline$C(13)-C(14)$ & $1.377(4)$ \\
\hline$C(14)-C(15)$ & $1.381(4)$ \\
\hline$C(16)-C(17)$ & $1.531(4)$ \\
\hline$C(16)-C(18)$ & $1.534(3)$ \\
\hline $\mathrm{C}(16)-\mathrm{C}(19)$ & $1.545(3)$ \\
\hline $\mathrm{C}(16)-\mathrm{Si}(1)$ & $1.906(2)$ \\
\hline$C(20)-C(25)$ & $1.395(4)$ \\
\hline $\mathrm{C}(20)-\mathrm{C}(21)$ & $1.402(3)$ \\
\hline $\mathrm{C}(20)-\mathrm{Si}(1)$ & $1.881(2)$ \\
\hline $\mathrm{C}(21)-\mathrm{C}(22)$ & $1.390(4)$ \\
\hline $\mathrm{C}(22)-\mathrm{C}(23)$ & $1.373(4)$ \\
\hline$C(23)-C(24)$ & $1.381(5)$ \\
\hline$C(24)-C(25)$ & $1.384(4)$ \\
\hline $\mathrm{C}(26)-\mathrm{C}(31)$ & $1.384(4)$ \\
\hline$C(26)-C(27)$ & $1.387(4)$ \\
\hline $\mathrm{C}(26)-\mathrm{N}(1)$ & $1.421(3)$ \\
\hline
\end{tabular}




\begin{tabular}{|c|c|}
\hline$C(27)-C(28)$ & $1.386(4)$ \\
\hline$C(28)-C(29)$ & $1.378(5)$ \\
\hline$C(29)-C(30)$ & $1.369(5)$ \\
\hline $\mathrm{C}(30)-\mathrm{C}(31)$ & $1.395(4)$ \\
\hline $\mathrm{O}(1)-\mathrm{C}(1)-\mathrm{N}(1)$ & $124.9(2)$ \\
\hline $\mathrm{O}(1)-\mathrm{C}(1)-\mathrm{C}(2)$ & $121.4(2)$ \\
\hline $\mathrm{N}(1)-\mathrm{C}(1)-\mathrm{C}(2)$ & $113.7(2)$ \\
\hline $\mathrm{O}(2)-\mathrm{C}(2)-\mathrm{C}(3)$ & $59.11(15)$ \\
\hline $\mathrm{O}(2)-\mathrm{C}(2)-\mathrm{C}(1)$ & $114.2(2)$ \\
\hline$C(3)-C(2)-C(1)$ & 119.1(2) \\
\hline $\mathrm{O}(2)-\mathrm{C}(3)-\mathrm{C}(2)$ & $58.59(16)$ \\
\hline $\mathrm{O}(2)-\mathrm{C}(3)-\mathrm{C}(4)$ & $117.6(2)$ \\
\hline$C(2)-C(3)-C(4)$ & $122.5(2)$ \\
\hline$C(5)-C(4)-C(3)$ & $118.8(2)$ \\
\hline$C(5)-C(4)-S i(1)$ & $125.46(19)$ \\
\hline $\mathrm{C}(3)-\mathrm{C}(4)-\mathrm{Si}(1)$ & $115.52(17)$ \\
\hline$C(4)-C(5)-C(6)$ & $126.7(3)$ \\
\hline$C(7)-C(6)-C(5)$ & 114.1(3) \\
\hline$C(8)-C(7)-C(6)$ & $124.4(5)$ \\
\hline $\mathrm{C}(7)-\mathrm{C}(8)-\mathrm{C}(9)$ & $128.2(5)$ \\
\hline$C(11)-C(10)-C(15)$ & $116.6(2)$ \\
\hline $\mathrm{C}(11)-\mathrm{C}(10)-\mathrm{Si}(1)$ & $123.97(19)$ \\
\hline$C(15)-C(10)-S i(1)$ & $119.45(19)$ \\
\hline$C(12)-C(11)-C(10)$ & 121.7(3) \\
\hline $\mathrm{C}(13)-\mathrm{C}(12)-\mathrm{C}(11)$ & $120.5(3)$ \\
\hline$C(12)-C(13)-C(14)$ & $119.4(3)$ \\
\hline$C(13)-C(14)-C(15)$ & $120.2(3)$ \\
\hline$C(14)-C(15)-C(10)$ & $121.7(3)$ \\
\hline $\mathrm{C}(17)-\mathrm{C}(16)-\mathrm{C}(18)$ & $108.8(2)$ \\
\hline$C(17)-C(16)-C(19)$ & $108.0(2)$ \\
\hline $\mathrm{C}(18)-\mathrm{C}(16)-\mathrm{C}(19)$ & $107.8(2)$ \\
\hline$C(17)-C(16)-\operatorname{Si}(1)$ & $110.65(17)$ \\
\hline$C(18)-C(16)-\operatorname{Si}(1)$ & $113.72(17)$ \\
\hline$C(19)-C(16)-\operatorname{Si}(1)$ & $107.70(16)$ \\
\hline$C(25)-C(20)-C(21)$ & $116.8(2)$ \\
\hline
\end{tabular}




$\begin{array}{lc}\mathrm{C}(25)-\mathrm{C}(20)-\mathrm{Si}(1) & 119.09(19) \\ \mathrm{C}(21)-\mathrm{C}(20)-\mathrm{Si}(1) & 124.0(2) \\ \mathrm{C}(22)-\mathrm{C}(21)-\mathrm{C}(20) & 121.2(3) \\ \mathrm{C}(23)-\mathrm{C}(22)-\mathrm{C}(21) & 120.3(3) \\ \mathrm{C}(22)-\mathrm{C}(23)-\mathrm{C}(24) & 119.8(3) \\ \mathrm{C}(23)-\mathrm{C}(24)-\mathrm{C}(25) & 119.8(3) \\ \mathrm{C}(24)-\mathrm{C}(25)-\mathrm{C}(20) & 122.0(3) \\ \mathrm{C}(31)-\mathrm{C}(26)-\mathrm{C}(27) & 119.9(2) \\ \mathrm{C}(31)-\mathrm{C}(26)-\mathrm{N}(1) & 117.7(2) \\ \mathrm{C}(27)-\mathrm{C}(26)-\mathrm{N}(1) & 122.4(2) \\ \mathrm{C}(28)-\mathrm{C}(27)-\mathrm{C}(26) & 119.2(3) \\ \mathrm{C}(29)-\mathrm{C}(28)-\mathrm{C}(27) & 121.1(3) \\ \mathrm{C}(30)-\mathrm{C}(29)-\mathrm{C}(28) & 119.6(3) \\ \mathrm{C}(29)-\mathrm{C}(30)-\mathrm{C}(31) & 120.2(3) \\ \mathrm{C}(26)-\mathrm{C}(31)-\mathrm{C}(30) & 119.9(3) \\ \mathrm{C}(1)-\mathrm{N}(1)-\mathrm{C}(26) & 127.8(2) \\ \mathrm{C}(2)-\mathrm{O}(2)-\mathrm{C}(3) & 62.30(16) \\ \mathrm{C}(20)-\mathrm{Si}(1)-\mathrm{C}(4) & 108.80(11) \\ \mathrm{C}(20)-\mathrm{Si}(1)-\mathrm{C}(10) & 113.41(11) \\ \mathrm{C}(4)-\mathrm{Si}(1)-\mathrm{C}(10) & 104.02(10) \\ \mathrm{C}(20)-\mathrm{Si}(1)-\mathrm{C}(16) & 107.31(10) \\ \mathrm{C}(4)-\mathrm{Si}(1)-\mathrm{C}(16) & 113.28(11) \\ \mathrm{C}(10)-\mathrm{Si}(1)-\mathrm{C}(16) & 110.13(10) \\ & \end{array}$

Symmetry transformations used to generate equivalent atoms: 
Table 4. Anisotropic displacement parameters $\left(\AA^{2} \times 10^{3}\right)$ for ya58sad. The anisotropic displacement factor exponent takes the form: $-2 \mathrm{p}^{2}\left[\mathrm{~h}^{2} \mathrm{a}^{* 2} \mathrm{U}^{11}+\ldots+2 \mathrm{~h} \mathrm{k} \mathrm{a}^{*} \mathrm{~b}^{*} \mathrm{U}^{12}\right]$

\begin{tabular}{|c|c|c|c|c|c|c|}
\hline & $\mathrm{U}^{11}$ & $\mathrm{U}^{22}$ & $\mathrm{U}^{33}$ & $\mathrm{U}^{23}$ & $\mathrm{U}^{13}$ & $\mathrm{U}^{12}$ \\
\hline $\mathrm{C}(1)$ & $25(1)$ & $31(1)$ & $23(1)$ & $1(1)$ & $2(1)$ & $-2(1)$ \\
\hline $\mathrm{C}(2)$ & $25(1)$ & $31(1)$ & $29(1)$ & $-3(1)$ & $2(1)$ & $0(1)$ \\
\hline$C(3)$ & $26(1)$ & $33(1)$ & $31(1)$ & $-2(1)$ & $3(1)$ & $-3(1)$ \\
\hline$C(4)$ & $23(1)$ & $27(1)$ & $25(1)$ & $-1(1)$ & $6(1)$ & $-1(1)$ \\
\hline$C(5)$ & $29(1)$ & $33(1)$ & $31(1)$ & $-1(1)$ & $7(1)$ & $2(1)$ \\
\hline$C(6)$ & $43(2)$ & $38(2)$ & $51(2)$ & $1(1)$ & $10(1)$ & $15(1)$ \\
\hline $\mathrm{C}(7)$ & $172(5)$ & $49(2)$ & $94(3)$ & $23(2)$ & $52(3)$ & $48(3)$ \\
\hline $\mathrm{C}(8)$ & 194(7) & $64(3)$ & $157(5)$ & $53(3)$ & $109(5)$ & $41(3)$ \\
\hline$C(10)$ & $24(1)$ & $29(1)$ & $25(1)$ & $-2(1)$ & $1(1)$ & $-2(1)$ \\
\hline $\mathrm{C}(11)$ & $31(2)$ & $44(2)$ & $56(2)$ & $17(1)$ & $-9(1)$ & $-6(1)$ \\
\hline$C(12)$ & $31(2)$ & $56(2)$ & $82(2)$ & $18(2)$ & $-20(2)$ & $-2(2)$ \\
\hline$C(13)$ & $23(1)$ & $50(2)$ & $63(2)$ & $-5(2)$ & $-2(1)$ & $-7(1)$ \\
\hline$C(14)$ & $36(2)$ & $39(2)$ & $50(2)$ & $1(1)$ & $4(1)$ & $-12(1)$ \\
\hline$C(15)$ & $29(1)$ & $34(1)$ & $39(1)$ & $4(1)$ & $-4(1)$ & $-2(1)$ \\
\hline$C(16)$ & $27(1)$ & $29(1)$ & $25(1)$ & $-2(1)$ & $-1(1)$ & $2(1)$ \\
\hline$C(17)$ & $36(2)$ & $43(2)$ & $36(1)$ & $-2(1)$ & $-9(1)$ & $-7(1)$ \\
\hline$C(18)$ & $38(2)$ & $37(1)$ & $26(1)$ & $3(1)$ & $4(1)$ & $5(1)$ \\
\hline$C(19)$ & $44(2)$ & $35(1)$ & $34(1)$ & $-6(1)$ & $-1(1)$ & $9(1)$ \\
\hline$C(20)$ & $27(1)$ & $29(1)$ & $23(1)$ & $3(1)$ & $-4(1)$ & $-7(1)$ \\
\hline$C(21)$ & $34(2)$ & $32(1)$ & $34(1)$ & $4(1)$ & $-6(1)$ & $-2(1)$ \\
\hline $\mathrm{C}(22)$ & $50(2)$ & $34(1)$ & $44(2)$ & $15(1)$ & $-18(1)$ & $-11(1)$ \\
\hline$C(23)$ & $42(2)$ & $52(2)$ & $44(2)$ & $24(1)$ & $-8(1)$ & $-19(2)$ \\
\hline$C(24)$ & $34(2)$ & $57(2)$ & $36(2)$ & $12(1)$ & $3(1)$ & $-12(1)$ \\
\hline$C(25)$ & $31(1)$ & $36(1)$ & $30(1)$ & $4(1)$ & $0(1)$ & $-6(1)$ \\
\hline$C(26)$ & $33(1)$ & $25(1)$ & $23(1)$ & $-1(1)$ & $4(1)$ & $-7(1)$ \\
\hline$C(27)$ & $38(2)$ & $38(1)$ & $33(1)$ & $-3(1)$ & $-1(1)$ & $-5(1)$ \\
\hline$C(28)$ & $55(2)$ & $45(2)$ & $34(2)$ & $-8(1)$ & $-5(1)$ & $-13(2)$ \\
\hline$C(29)$ & $77(2)$ & $35(2)$ & $35(2)$ & $-11(1)$ & $5(2)$ & $-3(2)$ \\
\hline $\mathrm{C}(30)$ & $53(2)$ & $37(2)$ & $43(2)$ & $-4(1)$ & $11(2)$ & $10(1)$ \\
\hline$C(31)$ & $37(2)$ & $33(1)$ & $33(1)$ & $-2(1)$ & $4(1)$ & $2(1)$ \\
\hline $\mathrm{N}(1)$ & $22(1)$ & $31(1)$ & $29(1)$ & $-5(1)$ & $-1(1)$ & $-4(1)$ \\
\hline
\end{tabular}




\begin{tabular}{lcccccc}
$\mathrm{O}(1)$ & $23(1)$ & $48(1)$ & $34(1)$ & $-7(1)$ & $2(1)$ & $-6(1)$ \\
$\mathrm{O}(2)$ & $44(1)$ & $50(1)$ & $34(1)$ & $-9(1)$ & $-7(1)$ & $21(1)$ \\
$\mathrm{Si}(1)$ & $21(1)$ & $24(1)$ & $23(1)$ & $2(1)$ & $0(1)$ & $-1(1)$ \\
$\mathrm{C}(9)$ & $186(6)$ & $45(2)$ & $84(3)$ & $12(2)$ & $40(3)$ & $9(3)$ \\
\hline
\end{tabular}


Table 5. Hydrogen coordinates ( $\left.\mathrm{x} 10^{4}\right)$ and isotropic displacement parameters $\left(\AA^{2} \mathrm{x} 10^{3}\right)$ for ya58sad.

\begin{tabular}{|c|c|c|c|c|}
\hline & $\mathrm{x}$ & $\mathrm{y}$ & $\mathrm{z}$ & $\mathrm{U}(\mathrm{eq})$ \\
\hline $\mathrm{H}(6 \mathrm{~A})$ & 2907 & 410 & 3560 & 53 \\
\hline $\mathrm{H}(6 \mathrm{~B})$ & 4389 & 653 & 3745 & 53 \\
\hline $\mathrm{H}(7 \mathrm{~A})$ & 3632 & -227 & 4172 & 126 \\
\hline $\mathrm{H}(7 \mathrm{~B})$ & 4043 & 341 & 4532 & 126 \\
\hline $\mathrm{H}(8 \mathrm{~A})$ & 1419 & 20 & 4319 & 166 \\
\hline $\mathrm{H}(8 \mathrm{~B})$ & 1952 & 506 & 4730 & 166 \\
\hline $\mathrm{H}(17 \mathrm{~A})$ & 3079 & 3008 & 4858 & 57 \\
\hline $\mathrm{H}(17 \mathrm{~B})$ & 2992 & 3208 & 4271 & 57 \\
\hline $\mathrm{H}(17 \mathrm{C})$ & 3552 & 2526 & 4424 & 57 \\
\hline $\mathrm{H}(18 \mathrm{~A})$ & 1968 & 1778 & 4857 & 50 \\
\hline $\mathrm{H}(18 \mathrm{~B})$ & 332 & 1896 & 4858 & 50 \\
\hline $\mathrm{H}(18 \mathrm{C})$ & 1309 & 2289 & 5232 & 50 \\
\hline $\mathrm{H}(19 \mathrm{~A})$ & 585 & 3296 & 4951 & 57 \\
\hline $\mathrm{H}(19 \mathrm{~B})$ & -549 & 2987 & 4584 & 57 \\
\hline $\mathrm{H}(19 \mathrm{C})$ & 539 & 3482 & 4361 & 57 \\
\hline $\mathrm{H}(22)$ & 1225 & 4346 & 2910 & 51 \\
\hline $\mathrm{H}(31)$ & 4987 & -242 & 1980 & 41 \\
\hline $\mathrm{H}(2)$ & $2740(20)$ & $1451(10)$ & $2682(8)$ & $15(6)$ \\
\hline $\mathrm{H}(3)$ & $690(30)$ & $832(12)$ & $3267(9)$ & $25(6)$ \\
\hline $\mathrm{H}(5)$ & $3180(30)$ & $1461(12)$ & $4234(10)$ & $30(7)$ \\
\hline $\mathrm{H}(27)$ & $1080(30)$ & $-22(13)$ & $1488(11)$ & $40(8)$ \\
\hline $\mathrm{H}(21)$ & $270(30)$ & $3627(12)$ & $3463(10)$ & $33(7)$ \\
\hline $\mathrm{H}(13)$ & $-4630(40)$ & $1743(15)$ & $3591(12)$ & $60(10)$ \\
\hline $\mathrm{H}(14)$ & $-3180(30)$ & $1117(16)$ & $4049(12)$ & $57(10)$ \\
\hline $\mathrm{H}(15)$ & $-900(30)$ & $1356(14)$ & $4166(11)$ & $42(8)$ \\
\hline $\mathrm{H}(25)$ & $3330(30)$ & $2417(13)$ & $3133(10)$ & $36(8)$ \\
\hline $\mathrm{H}(28)$ & $1400(30)$ & $-772(14)$ & $851(12)$ & $49(9)$ \\
\hline $\mathrm{H}(29)$ & $3520(40)$ & $-1308(16)$ & $804(13)$ & $64(10)$ \\
\hline $\mathrm{H}(11)$ & $-1420(40)$ & $2930(16)$ & $3305(13)$ & $65(10)$ \\
\hline $\mathrm{H}(30)$ & $5360(30)$ & $-1011(15)$ & $1364(12)$ & $52(9)$ \\
\hline
\end{tabular}




\begin{tabular}{lclll}
$\mathrm{H}(23)$ & $3260(30)$ & $4092(14)$ & $2472(12)$ & $46(8)$ \\
$\mathrm{H}(24)$ & $4290(40)$ & $3144(15)$ & $2596(12)$ & $57(10)$ \\
$\mathrm{H}(12)$ & $-3780(40)$ & $2656(16)$ & $3216(13)$ & $65(10)$ \\
$\mathrm{H}(1 \mathrm{~N})$ & $3580(30)$ & $563(13)$ & $2272(10)$ & $32(8)$ \\
$\mathrm{H}(9 \mathrm{~A})$ & 2274 & -776 & 4856 & 158 \\
$\mathrm{H}(9 \mathrm{~B})$ & 943 & -393 & 5044 & 158 \\
$\mathrm{H}(9 \mathrm{C})$ & 2434 & -238 & 5278 & 158 \\
\hline
\end{tabular}


Table 6. Torsion angles $\left[{ }^{\circ}\right]$ for ya58sad.

\begin{tabular}{|c|c|}
\hline $\mathrm{O}(1)-\mathrm{C}(1)-\mathrm{C}(2)-\mathrm{O}(2)$ & $-15.3(3)$ \\
\hline $\mathrm{N}(1)-\mathrm{C}(1)-\mathrm{C}(2)-\mathrm{O}(2)$ & $165.4(2)$ \\
\hline $\mathrm{O}(1)-\mathrm{C}(1)-\mathrm{C}(2)-\mathrm{C}(3)$ & $51.5(3)$ \\
\hline $\mathrm{N}(1)-\mathrm{C}(1)-\mathrm{C}(2)-\mathrm{C}(3)$ & $-127.9(2)$ \\
\hline $\mathrm{C}(1)-\mathrm{C}(2)-\mathrm{C}(3)-\mathrm{O}(2)$ & $-102.4(2)$ \\
\hline $\mathrm{O}(2)-\mathrm{C}(2)-\mathrm{C}(3)-\mathrm{C}(4)$ & $-104.8(3)$ \\
\hline $\mathrm{C}(1)-\mathrm{C}(2)-\mathrm{C}(3)-\mathrm{C}(4)$ & $152.9(2)$ \\
\hline $\mathrm{O}(2)-\mathrm{C}(3)-\mathrm{C}(4)-\mathrm{C}(5)$ & $-154.7(2)$ \\
\hline$C(2)-C(3)-C(4)-C(5)$ & $-86.1(3)$ \\
\hline $\mathrm{O}(2)-\mathrm{C}(3)-\mathrm{C}(4)-\mathrm{Si}(1)$ & $30.3(3)$ \\
\hline$C(2)-C(3)-C(4)-S i(1)$ & $99.0(2)$ \\
\hline$C(3)-C(4)-C(5)-C(6)$ & $0.2(4)$ \\
\hline $\mathrm{Si}(1)-\mathrm{C}(4)-\mathrm{C}(5)-\mathrm{C}(6)$ & $174.6(2)$ \\
\hline$C(4)-C(5)-C(6)-C(7)$ & $-125.7(4)$ \\
\hline$C(5)-C(6)-C(7)-C(8)$ & $49.2(7)$ \\
\hline$C(6)-C(7)-C(8)-C(9)$ & $171.0(5)$ \\
\hline$C(15)-C(10)-C(11)-C(12)$ & $-0.8(5)$ \\
\hline $\mathrm{Si}(1)-\mathrm{C}(10)-\mathrm{C}(11)-\mathrm{C}(12)$ & $177.4(3)$ \\
\hline $\mathrm{C}(10)-\mathrm{C}(11)-\mathrm{C}(12)-\mathrm{C}(13)$ & $0.5(6)$ \\
\hline $\mathrm{C}(11)-\mathrm{C}(12)-\mathrm{C}(13)-\mathrm{C}(14)$ & $0.4(6)$ \\
\hline $\mathrm{C}(12)-\mathrm{C}(13)-\mathrm{C}(14)-\mathrm{C}(15)$ & $-0.8(5)$ \\
\hline$C(13)-C(14)-C(15)-C(10)$ & $0.4(5)$ \\
\hline$C(11)-C(10)-C(15)-C(14)$ & $0.4(4)$ \\
\hline $\mathrm{Si}(1)-\mathrm{C}(10)-\mathrm{C}(15)-\mathrm{C}(14)$ & $-178.0(2)$ \\
\hline$C(25)-C(20)-C(21)-C(22)$ & $1.9(4)$ \\
\hline $\mathrm{Si}(1)-\mathrm{C}(20)-\mathrm{C}(21)-\mathrm{C}(22)$ & $177.5(2)$ \\
\hline$C(20)-C(21)-C(22)-C(23)$ & $-1.4(4)$ \\
\hline $\mathrm{C}(21)-\mathrm{C}(22)-\mathrm{C}(23)-\mathrm{C}(24)$ & $-0.2(4)$ \\
\hline $\mathrm{C}(22)-\mathrm{C}(23)-\mathrm{C}(24)-\mathrm{C}(25)$ & $1.4(4)$ \\
\hline $\mathrm{C}(23)-\mathrm{C}(24)-\mathrm{C}(25)-\mathrm{C}(20)$ & $-0.9(4)$ \\
\hline$C(21)-C(20)-C(25)-C(24)$ & $-0.7(4)$ \\
\hline $\mathrm{Si}(1)-\mathrm{C}(20)-\mathrm{C}(25)-\mathrm{C}(24)$ & $-176.6(2)$ \\
\hline $\mathrm{C}(31)-\mathrm{C}(26)-\mathrm{C}(27)-\mathrm{C}(28)$ & $-0.1(4)$ \\
\hline $\mathrm{N}(1)-\mathrm{C}(26)-\mathrm{C}(27)-\mathrm{C}(28)$ & $178.1(2)$ \\
\hline
\end{tabular}




\begin{tabular}{|c|c|}
\hline$C(26)-C(27)-C(28)-C(29)$ & $-0.3(4)$ \\
\hline $\mathrm{C}(27)-\mathrm{C}(28)-\mathrm{C}(29)-\mathrm{C}(30)$ & $0.4(5)$ \\
\hline$C(28)-C(29)-C(30)-C(31)$ & $-0.2(5)$ \\
\hline$C(27)-C(26)-C(31)-C(30)$ & $0.4(4)$ \\
\hline $\mathrm{N}(1)-\mathrm{C}(26)-\mathrm{C}(31)-\mathrm{C}(30)$ & $-177.9(2)$ \\
\hline$C(29)-C(30)-C(31)-C(26)$ & $-0.2(4)$ \\
\hline $\mathrm{O}(1)-\mathrm{C}(1)-\mathrm{N}(1)-\mathrm{C}(26)$ & $4.7(4)$ \\
\hline $\mathrm{C}(2)-\mathrm{C}(1)-\mathrm{N}(1)-\mathrm{C}(26)$ & $-176.0(2)$ \\
\hline $\mathrm{C}(31)-\mathrm{C}(26)-\mathrm{N}(1)-\mathrm{C}(1)$ & $-160.8(2)$ \\
\hline $\mathrm{C}(27)-\mathrm{C}(26)-\mathrm{N}(1)-\mathrm{C}(1)$ & $20.9(4)$ \\
\hline $\mathrm{C}(1)-\mathrm{C}(2)-\mathrm{O}(2)-\mathrm{C}(3)$ & $110.6(2)$ \\
\hline $\mathrm{C}(4)-\mathrm{C}(3)-\mathrm{O}(2)-\mathrm{C}(2)$ & $113.1(2)$ \\
\hline $\mathrm{C}(25)-\mathrm{C}(20)-\mathrm{Si}(1)-\mathrm{C}(4)$ & $-18.6(2)$ \\
\hline $\mathrm{C}(21)-\mathrm{C}(20)-\mathrm{Si}(1)-\mathrm{C}(4)$ & $165.9(2)$ \\
\hline $\mathrm{C}(25)-\mathrm{C}(20)-\mathrm{Si}(1)-\mathrm{C}(10)$ & $-133.80(19)$ \\
\hline$C(21)-C(20)-S i(1)-C(10)$ & $50.7(2)$ \\
\hline $\mathrm{C}(25)-\mathrm{C}(20)-\mathrm{Si}(1)-\mathrm{C}(16)$ & $104.4(2)$ \\
\hline$C(21)-C(20)-S i(1)-C(16)$ & $-71.2(2)$ \\
\hline $\mathrm{C}(5)-\mathrm{C}(4)-\mathrm{Si}(1)-\mathrm{C}(20)$ & $107.4(2)$ \\
\hline $\mathrm{C}(3)-\mathrm{C}(4)-\mathrm{Si}(1)-\mathrm{C}(20)$ & $-78.05(19)$ \\
\hline $\mathrm{C}(5)-\mathrm{C}(4)-\mathrm{Si}(1)-\mathrm{C}(10)$ & $-131.5(2)$ \\
\hline $\mathrm{C}(3)-\mathrm{C}(4)-\mathrm{Si}(1)-\mathrm{C}(10)$ & $43.12(19)$ \\
\hline $\mathrm{C}(5)-\mathrm{C}(4)-\mathrm{Si}(1)-\mathrm{C}(16)$ & $-11.9(3)$ \\
\hline$C(3)-C(4)-S i(1)-C(16)$ & $162.69(17)$ \\
\hline $\mathrm{C}(11)-\mathrm{C}(10)-\mathrm{Si}(1)-\mathrm{C}(20)$ & $-13.9(3)$ \\
\hline $\mathrm{C}(15)-\mathrm{C}(10)-\mathrm{Si}(1)-\mathrm{C}(20)$ & $164.32(19)$ \\
\hline $\mathrm{C}(11)-\mathrm{C}(10)-\mathrm{Si}(1)-\mathrm{C}(4)$ & $-131.9(2)$ \\
\hline $\mathrm{C}(15)-\mathrm{C}(10)-\mathrm{Si}(1)-\mathrm{C}(4)$ & $46.3(2)$ \\
\hline$C(11)-C(10)-S i(1)-C(16)$ & $106.4(2)$ \\
\hline $\mathrm{C}(15)-\mathrm{C}(10)-\mathrm{Si}(1)-\mathrm{C}(16)$ & $-75.4(2)$ \\
\hline$C(17)-C(16)-S i(1)-C(20)$ & $-44.0(2)$ \\
\hline $\mathrm{C}(18)-\mathrm{C}(16)-\mathrm{Si}(1)-\mathrm{C}(20)$ & $-166.77(17)$ \\
\hline $\mathrm{C}(19)-\mathrm{C}(16)-\mathrm{Si}(1)-\mathrm{C}(20)$ & $73.88(19)$ \\
\hline $\mathrm{C}(17)-\mathrm{C}(16)-\mathrm{Si}(1)-\mathrm{C}(4)$ & $76.1(2)$ \\
\hline $\mathrm{C}(18)-\mathrm{C}(16)-\mathrm{Si}(1)-\mathrm{C}(4)$ & $-46.7(2)$ \\
\hline $\mathrm{C}(19)-\mathrm{C}(16)-\mathrm{Si}(1)-\mathrm{C}(4)$ & $-166.01(16)$ \\
\hline
\end{tabular}


C(17)-C(16)-Si(1)-C(10)

$-167.88(17)$

$\mathrm{C}(18)-\mathrm{C}(16)-\mathrm{Si}(1)-\mathrm{C}(10)$

69.3(2)

$\mathrm{C}(19)-\mathrm{C}(16)-\mathrm{Si}(1)-\mathrm{C}(10)$

$-50.00(19)$

Symmetry transformations used to generate equivalent atoms: 
Table 7. Hydrogen bonds for ya58sad [ $\left[\AA\right.$ and $^{\circ}$ ].

\begin{tabular}{lllll}
\hline D-H...A & $d(\mathrm{D}-\mathrm{H})$ & $\mathrm{d}(\mathrm{H} \ldots \mathrm{A})$ & $\mathrm{d}(\mathrm{D} \ldots \mathrm{A})$ & $<(\mathrm{DHA})$ \\
\hline $\mathrm{N}(1)-\mathrm{H}(1 \mathrm{~N}) \ldots \mathrm{O}(1) \# 1$ & $0.82(3)$ & $2.26(3)$ & $3.021(3)$ & $154(3)$ \\
\hline
\end{tabular}

Symmetry transformations used to generate equivalent atoms:

$\# 1 \mathrm{x}+1 / 2, \mathrm{y},-\mathrm{z}+1 / 2$ 